\section{SANDIA REPORT}

SAND96-2254 • UC-706

Unlimited Release

Printed September 1996
RECEIVED

OCT 151996

OSTI

\title{
Measurements of Transient Electromagnetic Propagation Through Concrete and Sand
}

John F. Aurand

\section{Prepared by}

Sandia National Laboratories

Albuquerque, New Mexico 87185 and Livermore, California 94550

for the United States Department of Energy

under Contract DE-AC04-94AL85000

Approved for public release; distribution is unlimited.

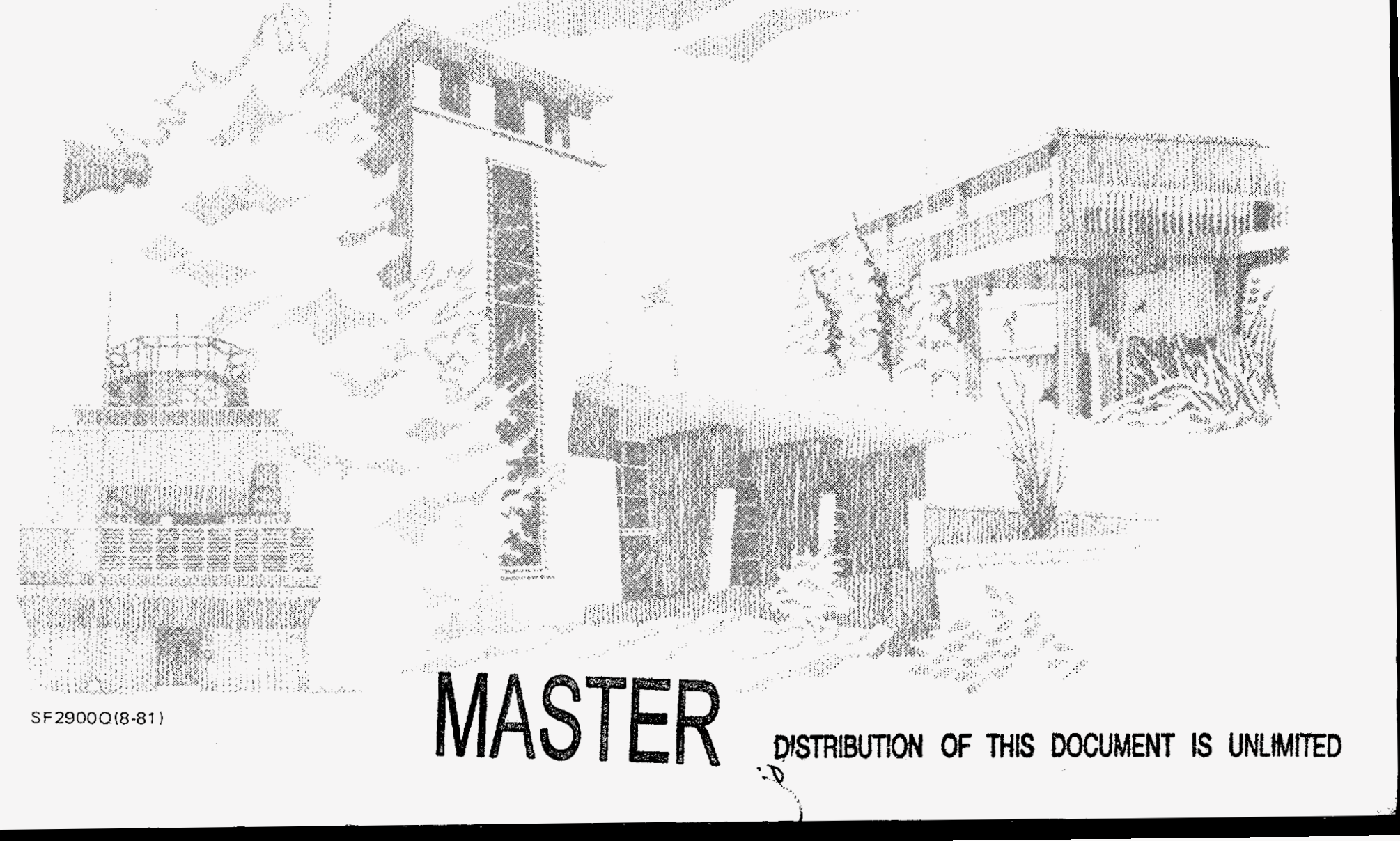


Issued by Sandia National Laboratories, operated for the United States Department of Energy by Sandia Corporation.

NOTICE: This report was prepared as an account of work sponsored by an agency of the United States Government. Neither the United States Government nor any agency thereof, nor any of their employees, nor any of their contractors, subcontractors, or their employees, makes any warranty, express or implied, or assumes any legal liability or responsibility for the accuracy, completeness, or usefulness of any information, apparatus, product, or process disclosed, or represents that its use would not infringe privately owned rights. Reference herein to any specific commercial product, process, or service by trade name, trademark, manufacturer, or otherwise, does not necessarily constitute or imply its endorsement, recommendation, or favoring by the United States Government, any agency thereof or any of their contractors or subcontractors. The views and opinions expressed herein do not necessarily state or reflect those of the United States Government, any agency thereof or any of their contractors.

Printed in the United States of America. This report has been reproduced directly from the best available copy.

Available to DOE and DOE contractors from

Office of Scientific and Technical Information

PO Box 62

Oak Ridge, TN 37831

Prices available from (615) 576-8401, FTS 626-8401

Available to the public from

National Technical Information Service

US Department of Commerce

5285 Port Royal Rd

Springfield, VA 22161

NTIS price codes

Printed copy: A05

Microfiche copy: A01 


\title{
Measurements of Transient Electromagnetic Propagation Through Concrete and Sand
}

\author{
John F. Aurand \\ High-Power Electromagnetics Department \\ Sandia National Laboratories \\ Albuquerque, NM 87185-1153
}

\begin{abstract}
This report is a summary of experimental measurements of transient electromagnetic propagation through a variety of media, with the goal of accurately characterizing the attenuation and dispersion of very short time-domain electromagnetic pulses through these materials. This included measurements through: temporary stacked walls of solid concrete cinder blocks; reinforced poured-concrete walls; and a 6-ft. thick sandbox. The measurement technique we developed for this work is a radiated-transmission approach for characterizing the in-situ complex permittivity of lossy dielectric layers. Our experimental objectives were to develop and implement accurate, repeatable, consistent measurements of single-pass time-domain propagation through representative earth media. Several different short-duration pulses were used for the incident radiation, with wideband spectral content from $300 \mathrm{MHz}$ to $20 \mathrm{GHz}$. We formulate a transfer function of the layer or wall under test, and compute the dielectric constant, loss tangent, attenuation constant, and time-domain impulse response of the layer. The attenuation for the media tested here increases steadily with frequency, and is a strong function of the moisture content. The time-domain pulse attenuation and dispersion are consistent with a lowpass-filtering effect of this attenuation loss versus frequency. This unique data should prove invaluable for modeling microwave propagation in earth materials.
\end{abstract}




\section{Acknowledgments}

Thanks are due to several people who have faithfully supported this work: Malcolm Buttram, my department manager; Bill Tucker (now retired) and Tom Hitchcock, Sandia program managers for the Munitions Technology DoD-DOE MOU; and Kwang Min, USAF Wright Laboratory Armament Directorate. I deeply appreciate your long-standing support and encouragement. 


\section{DISCLAIMER}

Portions of this document may be illegible in electronic image products. Images are produced from the best available original document. 


\section{Contents}

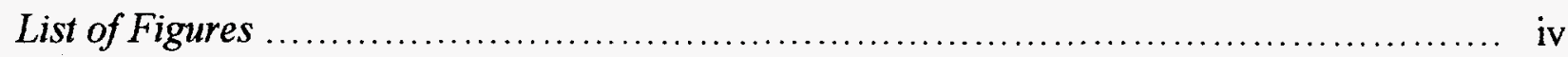

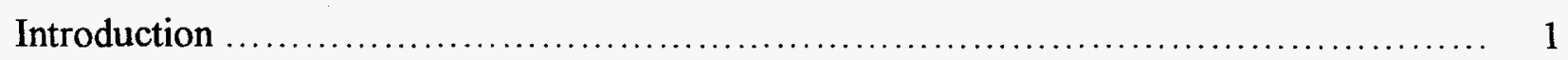

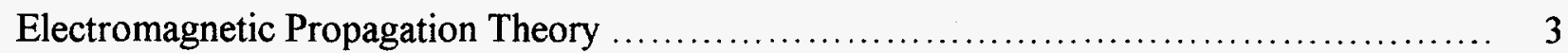

Electrical Properties of Earth Media $\quad 3$

Radiated Transmission Measurement Technique $\quad 7$

Low-Loss Analysis Method $\quad 13$

Series-1 Experiments ...................................................... 15

$\begin{array}{ll}\text { Temporary Concrete-Block Walls } & 16\end{array}$

Permanent Concrete Walls $\quad 18$

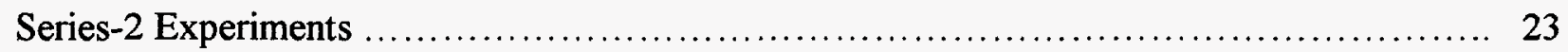

Sandia Labs Concrete Wall $\quad 24$

Wright Lab Concrete Wall $\quad 38$

$\begin{array}{ll}\text { Wright Lab Sandbox } & 48\end{array}$

Experimental Summary $\quad 56$

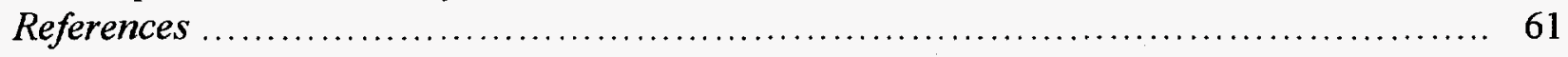

Appendix: TEM Horn Antenna with Dielectric Lens ................................ 65

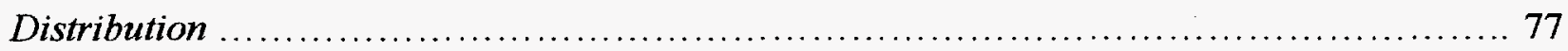




\section{List of Figures}

\section{Measurement Technique}

1. Short-Pulse Plane-Wave Propagation Configuration.

2. Bounce Diagram for Short-Pulse Propagation Measurements. 10

\section{Series-1 Experiments}

3. Experimental Configuration for Nylon-Slab Validation Measurements. 16

4. Experimental Configuration for Double-Layer Concrete-Block Wall. 17

5. Transmitting Hardware for Internal Sandia Concrete Wall. 20

6. Receiving Hardware for Internal Sandia Concrete Wall. 20

\section{Series-2 Experiments - Sandia Concrete Wall}

7. Block Diagram of 'Through' Setup for Sandia Concrete Wall.

8. Free-Space Reference and 'Through' Waveforms for 48-ps Pulse-Duration Measurement of Sandia Concrete Wall.

9. 62-ps Free-Space and 'Through' Waveforms for Sandia Concrete Wall.

10. 75-ps Free-Space and 'Through' Waveforms for Sandia Concrete Wall.

11. 112-ps Free-Space and 'Through' Waveforms for Sandia Concrete Wall.

12. Cross-Correlation Function for Four Datasets of Sandia Concrete Wall.

13. 48-ps Free-Space, Time-Shifted Free-Space, and 'Through' Waveforms for Sandia Concrete Wall.

14. Frequency-Domain Spectra of Free-Space and 'Through' Waveforms for Sandia Concrete Wall, with 48-ps System Pulse Duration.

15. Log-Magnitude of Transfer Function of Sandia Concrete Wall, for System Pulse Durations of $48 \mathrm{ps}, 62 \mathrm{ps}, 75 \mathrm{ps}$, and $112 \mathrm{ps}$.

16. Log-Magnitude of Vector Analyzer Free-Space and 'Through' $S_{21}(f)$ Measurements for Sandia Concrete Wall.

17. Bandpass Impulse 'Time-Domain' Data of Vector Analyzer Free-Space and 'Through' Measurements for Sandia Concrete Wall.

18. Comparison of Transfer Function Magnitude of Sandia Concrete Wall, for Time-Domain System (48-ps Pulse Duration) and Vector Analyzer System.

19. Impulse Response of Sandia Concrete Wall, for System Pulse Durations of 48 ps, $62 \mathrm{ps}, 75 \mathrm{ps}$, and $112 \mathrm{ps}$.

20. Dielectric Constant of Sandia Concrete Wall, Derived by Low-Loss Analysis Method from the Transfer Function of 48-ps Transient Data, Overlaid with Time-Domain Value from Maximum Cross-Correlation Lag.

21. Loss Tangent of Sandia Concrete Wall, Derived by Low-Loss Analysis Method from the Transfer Function of 48-ps Transient Data.

22. Attenuation Constant of Sandia Concrete Wall, Based on Series-2 Transient Data (48-ps System Pulse Duration) and on Series-1 Data. 


\section{Series-2 Experiments - Wright Concrete Wall}

23. Wright Lab Experimental Setup of Free-Space Reference Measurement for Characterization of Concrete Wall.

24. Block Diagram of Transmitter for Wright Lab Measurements.

25. Transmitter Setup for 'Through' Measurement of Concrete Wall at Wright Lab.

26. Receiver Setup for 'Through' Measurement of Concrete Wall at Wright Lab.

27. Receiver Setup with Four Vertical Scattering Wires Taped to Concrete Wall at Wright Lab.

28. Free-Space and 'Through' Waveforms for Wright Concrete Wall Using Fastest (48-ps) Impulse System.

29. 64-ps Impulse Free-Space and 'Through' Waveforms for Wright Concrete Wall.

30. 84-ps Impulse Free-Space and 'Through' Waveforms for Wright Concrete Wall.

31. 115-ps Impulse Free-Space and 'Through' Waveforms for Wright Concrete Wall.

32. 193-ps Impulse Free-Space and 'Through' Waveforms for Wright Concrete Wall.

33. Free-Space and 'Through' Waveforms for Wright Concrete Wall Using Bipolar System.

34. Log-Magnitude of Transfer Function of Wright Concrete Wall Using the Six System Excitation Pulses.

35. Impulse Response of Wright Concrete Wall Using the Six System Excitations.

36. Peak-Normalized and Time-Aligned Impulse Response of Wright Concrete Wall Using the 48-ps, 64-ps, and 84-ps Impulses and the Bipolar System Excitation.

37. Dielectric Constant of Wright Wall and Sandia Wall, Both Based on the Fastest Impulse Systems.

\section{Series-2 Experiments - Wright Lab Sandbox}

38. Wright Lab Sandbox, Showing Receiving Side.

39. Receiver Setup for 'Through' Measurement of Wright Lab Sandbox. 50

40. Experimental Setup for Free-Space Measurement of Wright Lab Sandbox. 51

41. Free-Space and 'Through' Acquired Waveforms for Wright Lab Sandbox. 52

42. Acquired and Modeled 'Through' Waveforms for Wright Lab Sandbox.

43. Processed Free-Space and Modeled 'Through' Waveforms for Wright Lab Sandbox.

44. Log-Magnitude of Vector Analyzer 'Through' $S_{21}(f)$ Measurements for Wright Lab Sandbox; Ungated Data and Time-Gated Data.

45. Comparison of Transfer Function Magnitude of Wright Lab Sandbox, for Impulse Time-Domain System and Time-Gated Vector Analyzer System.

46. Impulse Response of Wright Lab Sandbox, Based on Inverse Fourier Transform of Time-Domain Dataset.

\section{Series-2 Experiments - Summary}

47. Transfer Function Magnitude for 12 -in. Thick Sandia and Wright Concrete Walls, Based on Fastest Impulse Datasets.

48. Impulse Response for Sandia and Wright Concrete Walls, Based on Fastest Impulse Datasets. 
49. Attenuation Constant of Sandia and Wright Concrete Walls and Wright Lab Sandbox, Based on Fastest Impulse Datasets.

50. Percentage of Time-Domain Pulse Energy Propagated Through Sandia and Wright Concrete Walls, Based on Impulse Time-Domain Datasets.

\section{Appendix - TEM Horn Antenna}

A-1. Printed-Board Configuration of TEM Horn Antenna with Dielectric Lens.

A-2. Top Surface and Bottom Surface of Printed-Board Configuration of TEM Horn Antenna.

A-3. Solid-Plate Configuration of TEM Horn Antennas.

A-4. Top and Bottom Conductors of Solid-Plate Antenna Configuration.

A-5. Close-up View of Feedpoint Region of Solid-Plate Antenna Configuration.

A-6. Experimental Setup to Measure Boresight Time-Domain Response of Solid-Plate Antenna.

A-7. Block Diagram of Experimental Setup to Measure Boresight Time-Domain Response of Solid-Plate Antenna.

A-8. Boresight Time-Domain Response of Solid-Plate Antenna.

A-9. Time-Integrated 'Step-Equivalent' Boresight Time-Domain Response of Solid-Plate Antenna.

A-10. Experimental Setup to Measure Horizontal (H-Plane) Time-Domain Pattern of Solid-Plate TEM Horn Antenna.

A-11.Block Diagram of Experimental Setup to Measure H-Plane Time-Domain Pattern of Solid-Plate Antenna.

A-12. Received Waveforms for Time-Domain H-Plane Pattern of Solid-Plate Antenna.

A-13. Frequency-Domain H-Plane Pattern of Solid-Plate TEM Horn Antenna, Based on Spectral Ratio of Received Waveforms in Figure A-12. 


\section{Introduction}

This report is a summary of experimental measurements of transient electromagnetic propagation through a variety of media. The goal was to accurately characterize the attenuation and dispersion of very short pulse electromagnetic waves through these materials. We began this work in FY94, but performed most of it during FY95, in two series of experiments.

The Series-1 experiments included the following materials:

- nylon slabs (4 in. thick, for validation of the methodology);

- temporary stacked walls of solid concrete cinder-blocks (7.5-22.5 in. thick);

- an outdoor reinforced poured-concrete earth-berm wall (14 in. thick); and

- an interior reinforced poured-concrete wall (12 in. thick).

The Series-2 experiments included materials at two facilities:

- the same interior concrete wall (at Sandia/New Mexico); as well as

- an interior reinforced poured-concrete wall (12 in. thick, at USAF Wright Laboratory, Eglin AFB, FL); and

- a wood-walled sandbox (6 ft. thick, at Wright Lab).

The measurement technique we developed for this work is a radiated-transmission approach for characterizing the complex permittivity of lossy dielectric layers. It was designed for making in-situ measurements of building walls, earth-berm walls, etc. The system hardware was upgraded several times during this work, including antennas, pulse generators, and receiving equipment. A variety of short-duration pulses were used for the incident electromagnetic radiation, with wideband frequency content from UHF to $20 \mathrm{GHz}$. Corroborating swept-frequency measurements were also made with a vector network analyzer.

Our experimental objectives were to develop and implement accurate, repeatable, consistent measurements of single-pass time-domain propagation through these media. Note that the materials tested were meant to be representative only, so that this data should be taken as such the results herein are not exhaustive or thorough, but only meant to be representative of transient electromagnetic propagation through earth media.

This report describes the single-pass propagation model of plane-wave propagation through a lossy dielectric layer, our time-domain measurement technique, the Series-1 and Series-2 propagation measurements, and the resulting analysis. There is also an appendix describing the new TEM horn antennas with dielectric lens which we designed and built for the Series-2 experiments.

We formulate a transfer function of the layer or wall under test, and compute the dielectric constant, loss tangent, attenuation constant, and time-domain impulse response of the layer. In general for earth media, water content is the primary determiner of electrical properties. The attenuation for the media tested here increases steadily with frequency, and is a strong function of 
the moisture content. The time-domain pulse attenuation and dispersion are consistent with a lowpass-filtering effect of this attenuation loss versus frequency.

The single-pass propagation time-domain behavior has been quantified, and will be very useful in time-domain radar studies for ground-penetrating radar, free-space layered-material measurement systems, etc. Future work could include: time-domain backscatter (radiated reflection) studies to corroborate with the radiated transmission measurement approach; modeling of the data presented herein for system propagation studies; better/cleaner measurements of soil samples; comparison of single-pass transient propagation through layers with sinusoidal steady-state multiple-pass propagation behavior; examination of expected rebar shielding at lower frequencies; and joint time-frequency analysis of the transient data. 


\section{Electromagnetic Propagation Theory}

\section{Electrical Properties of Earth Media}

The electromagnetic theory for lossy dielectric media is found in a number of texts, including von Hippel (1954), King and Smith (1981), Nyfors and Vainikainen (1989), Miller (1986), Pozar (1990), Balanis (1989), Collin (1992), Ramo et al. (1965), Fuller and Wait (1976), Wait (1969), and Oughstun and Sherman (1994). In terms of the macroscopic properties of linear, homogeneous, isotropic non-ferromagnetic media, electromagnetic losses can be modeled by two physical processes: dielectric polarization loss, and conductivity loss. The dielectric loss represents electromagnetic energy loss due to bound-charge displacement currents set up within a material, whereas the conductivity loss is due to free-charge conduction currents set up within the material. Note that this analysis assumes free-space permeability; King and Smith (1981), Miller (1986), Fuller and Wait (1976), Wait (1969), and Gans et al. (1991) explain that this is approximately true for many earth/soil types.

In this theoretical discussion, we follow the typical convention of assuming sinusoidal steady-state field conditions. However, it should be pointed out that an active area of theoretical interest in electromagnetic propagation is concerned with the more general problem of time-dependent permittivity and constitutive relations for the electromagnetic fields. This issue is beyond the scope of this report, as well as the topic of joint time-frequency analysis, even though both may be worth pursuing in the future.

For a TEM (transverse electromagnetic) plane wave propagating in the $+z$ direction, we use the time-harmonic basis function of $E(f, z)=E(j \omega, z)=E_{0} e^{j \omega t} e^{-\gamma z}$, where the radian frequency is $\omega=2 \pi f, f$ is the frequency $(\mathrm{Hz})$, and the complex propagation constant is defined to be

$$
\gamma(f) \equiv \alpha(f)+j \beta(f)=j \omega \sqrt{\mu \varepsilon},
$$

with an attenuation constant of $\alpha(\mathrm{Np} / \mathrm{m})$, a phase constant of $\beta(\mathrm{rad} / \mathrm{m})$, a permittivity of $\varepsilon$, and a permeability of $\mu$. Note that $\mu=\mu_{r} \mu_{0} \cong \mu_{0}$, for non-magnetic media.

The dielectric polarization loss may be represented by a complex permittivity $\varepsilon(f) \equiv \varepsilon^{\prime}(f)-j \varepsilon^{\prime \prime}(f)$, where $\varepsilon^{\prime}=\varepsilon_{r} \varepsilon_{0}$ is the real permittivity, $\varepsilon_{r}$ is the relative permittivity or dielectric constant $(\geq 1)$, and $\varepsilon_{0}=8.854 \times 10^{-12} \mathrm{~F} / \mathrm{m}$ is the permittivity of free space. Note that some authors use $\varepsilon^{*}(f)$ to more carefully denote this complex permittivity formulation. Both the attenuation constant and phase constant are a function of the complex permittivity. The imaginary part of the complex permittivity is the dielectric loss factor, ' $\varepsilon$ ", and the resulting loss tangent is $p(f)=\tan \delta \equiv \varepsilon^{\prime \prime}(f) / \varepsilon^{\prime}(f)$. The complex permittivity may then be written $\varepsilon=\varepsilon^{\prime}(1-j p)$. 
The conductivity loss can be modeled by an additional imaginary term in the complex permittivity, $\varepsilon(f)=\varepsilon^{\prime}-j\left(\varepsilon^{\prime \prime}+\sigma / \omega\right)$, where $\sigma(f)$ is the macroscopic conductivity of the material of interest (in S/m). Then, following King and Smith (1981), Pozar (1990), Balanis (1989), Collin (1992), and Fuller and Wait (1976), the dielectric and conductivity losses may be combined in an effective loss tangent (which is $\geq 0$ ):

$$
p_{e}(f)=\frac{\varepsilon^{\prime \prime}+\sigma / \omega}{\varepsilon^{\prime}}=\frac{\varepsilon^{\prime \prime}}{\varepsilon^{\prime}}+\frac{\sigma}{\omega \varepsilon^{\prime}} .
$$

As the references point out, there is no way on the macroscopic scale to separate these loss effects at a given frequency. However, relative variations across the frequency spectrum are constrained by these types of losses, and the macroscopic frequency-dependent behavior hopefully can be used to deduce the particular contributions of each type within a given medium. For example, if the dielectric loss factor is constant over a certain frequency band, then its' loss tangent will be too; on the other hand, if the conductivity is constant with frequency, then its' loss tangent will drop with frequency.

The combined effective permittivity is then $\varepsilon=\varepsilon^{\prime}\left(1-j p_{e}\right)$. In terms of the relative complex effective permittivity, this yields our desired form of

$$
\varepsilon_{r e}(f)=\varepsilon_{r}(f)\left[1-j p_{e}(f)\right] \text {. }
$$

We thus have two parameters to characterize any subsurface material:

- the dielectric constant $\varepsilon_{r}(f)$; and

- the effective loss tangent $p_{e}(f)$.

The complex propagation constant is then expressible as

$$
\gamma(f)=\frac{j \omega}{c} \sqrt{\varepsilon_{r e}}=\frac{j \omega}{c} \sqrt{\varepsilon_{r}\left(1-j p_{e}\right)},
$$

with the speed of light $c=1 / \sqrt{\mu_{0} \varepsilon_{0}} \cong 3 \times 10^{8} \mathrm{~m} / \mathrm{s}$. In terms of TEM plane-wave propagation inside the material, the attenuation and phase constants in the exponential can be separated:

$$
E(f, z)=E_{0} e^{-\gamma(f) z}=E_{0} e^{-j \beta(f) z} e^{-\alpha(f) z} .
$$

The attenuation constant works out to be

$$
\alpha(f)=\frac{2 \pi f}{c}\left\{\frac{\varepsilon_{r}}{2}\left[\sqrt{1+p_{e}^{2}}-1\right]\right\}^{1 / 2} \mathrm{~Np} / \mathrm{m}
$$

and the phase constant becomes

$$
\beta(f)=\frac{2 \pi f}{c}\left\{\frac{\varepsilon_{r}}{2}\left[\sqrt{1+p_{e}^{2}}+1\right]\right\}^{1 / 2} \mathrm{rad} / \mathrm{m} .
$$

The attenuation of the field strength in $\mathrm{dB} / \mathrm{m}$ is simply $\alpha(\mathrm{dB} / \mathrm{m})=8.686 \alpha(\mathrm{Np} / \mathrm{m})$.

For electromagnetic waves in media, the remaining parameter to introduce is the concept of wave impedance. This is defined as the ratio of the electric field vector to the magnetic field vector: $\eta(f) \equiv|E(f)| /|H(f)| \Omega$, which for TEM waves is equal to the intrinsic impedance of the medium: $\eta(f)=\sqrt{\mu / \varepsilon}$. The free-space wave impedance is $\eta_{0} \equiv \sqrt{\mu_{0} / \varepsilon_{0}} \cong 376.73 \Omega$. Therefore, 
in a non-ferromagnetic material with complex permittivity $\varepsilon_{r e}(f)$, the complex wave impedance becomes

$$
\eta(f)=\frac{\eta_{0}}{\sqrt{\varepsilon_{r e}(f)}}=\frac{\eta_{0}}{\sqrt{\varepsilon_{r}(f)\left[1-j p_{e}(f)\right]}}
$$

Now consider empirical studies of soil properties. King and Smith (1981), Nyfors and Vainikainen (1989), and Fuller and Wait (1976) explain that most soils and rocks contain moisture, and that if the water content is large, the permittivity is determined more by the water dielectric properties than by the material itself. For frequencies below $1 \mathrm{MHz}$, the loss due to ionic conductivity is dominant. But water dipolar relaxation is apparent above $1 \mathrm{MHz}$, and becomes the significant loss mechanism above $1 \mathrm{GHz}$ (our main region of interest). In addition, subsurface media can in general be considered as mixtures of a variety of dielectric substances, each contributing to this effective permittivity.

There is a long history of soil measurements, with higher-frequency characterization only in the last decade or two. Hipp (1974) used transmission-line methods to measure two types of clay loam over a range of $30 \mathrm{MHz}$ to $4 \mathrm{GHz}$. Brock and Patitz (1993) describe curve-fit equations to Hipp's data, based on several Debye relaxation terms. Hoekstra and Delaney (1974) used both time-domain and frequency-domain methods to characterize a sand, a silt, and two clays over a range of $100 \mathrm{MHz}$ to $26 \mathrm{GHz}$. They show a Cole-Cole relationship for the soil complex permittivity, with broader dispersion spectra than simple Debye behavior. They give a thorough discussion of the effects of temperature and moisture content on the different soil types, and point out that moisture content has a stronger influence on permittivity than the actual type of soil. Scott and Smith (1992) also stress the moisture dependence irrespective of soil type. This is borne out by the extensive literature dealing with in-situ electromagnetic methods for measuring soil moisture, relatively independent of soil type.

A pair of companion papers, Hallikainen et al. (1985) and Dobson et al. (1985), present soil permittivity behavior for $1.4-18 \mathrm{GHz}$, especially as a function of moisture content. Included is a comprehensive semiempirical mixing model for the complex permittivity. Then Peplinski et al. (1995) extended this work down in frequency to a range of $300 \mathrm{MHz}$ to $1.3 \mathrm{GHz}$. Calvet et al. (1995) describe surface backscatter measurements from soil in France at millimeter-wave frequencies $(24,36$, and $90 \mathrm{GHz})$, and compares the data with the model of Dobson et al. (1985) and a different dielectric mixing model given by Wang and Schmugge (1980). This last reference has data at 1.4 and $5 \mathrm{GHz}$. Gans et al. (1991) provide an in-depth discussion of this soil phenomenology, especially in the context of mine detection.

As an illustration of the wide range of attenuation possible for typical soils, consider two examples of published data, at a frequency of $1 \mathrm{GHz}$. The loss tangent is a strong function of the water content of the soil in question, so the two examples will be representative extremes. First, consider the data for typical clay-loam soil with moderate water content (about $10 \%$ by weight), as described in King and Smith (1981, pp. 427-429). At $1 \mathrm{GHz}$, the dielectric constant is about 10 , and the conductivity is about $0.1 \mathrm{~S} / \mathrm{m}$. This corresponds to a loss tangent of $0.18 \mathrm{at} 1 \mathrm{GHz}$, and yields an attenuation of $51 \mathrm{~dB} / \mathrm{m}$ at $1 \mathrm{GHz}$, a severe amount. Now consider the data for dry 
silica sand, as described in Gans et al. (1991, p. 5-16). The dielectric constant of the sand (2.8) and the loss tangent $(0.01)$ are much lower than those of the clay. As a result, this yields an attenuation of only $1.5 \mathrm{~dB} / \mathrm{m}$, which is very little loss.

Compared to the number of papers dealing with soil properties, the number devoted to concrete walls or floors is much more limited. We are interested in the attenuation through steel-reinforced concrete layers/walls for a variety of electromagnetic system concepts, and one of the motivating factors for the measurement of concrete walls in this report was the lack of data for frequencies above $1 \mathrm{GHz}$.

Some data are available about propagation through concrete floors inside buildings extensive measurements of indoor radio propagation have been made, but very few were careful enough to remove the effects of extraneous propagation paths (especially the diffraction of radio waves out through windows and then back in the windows of a different floor). Honcharenko et al. (1994) discuss various configurations of concrete floors including: precast concrete slabs; reinforced concrete, assumed to be poured in place with reinforcing steel bars and/or mesh; and concrete poured over corrugated steel panels. Typical average values of one-way attenuation through reinforced concrete floors are 9-13 dB for frequencies of $850-920 \mathrm{MHz}$, and $12 \mathrm{~dB}$ at $850 \mathrm{MHz}$ through precast concrete with rebar. Horikoshi et al. (1986) studied indoor propagation in a reinforced concrete building at $1290 \mathrm{MHz}$, and show attenuation values of $8.5 \mathrm{~dB}$ through walls, and 9.1-10 dB through floors. They also indicate that the reflection coefficient of the concrete walls (with 9-mm-dia. steel cables placed every $15 \mathrm{~cm}$ within) was 0.33-0.4. The corresponding equivalent dielectric constant was thus 3.9-5.4. This is consistent with our measurement data, as shown later on.

The electromagnetic attenuation is certainly proportional to the amount of metal in the concrete, as it contributes the most to shielding (especially at lower frequencies). The worst case would obviously be layers that have contiguous/continuous sheet metal shielding, which has much greater attenuation than through concrete walls with only steel reinforcing bars. Lafortune and Lecours (1990) include an extensive indoor attenuation modeling effort, and indicate a one-way loss of $22 \mathrm{~dB}$ at $900 \mathrm{MHz}$ for propagation through reinforced concrete and a suspended metal-tile ceiling. Patsiokas et al. (1986) have data at three bands for reinforced concrete on corrugated sheet metal; the average one-way path loss is $16 \mathrm{~dB}$ at $150 \mathrm{MHz}, 25 \mathrm{~dB}$ at $450 \mathrm{MHz}$, and $28 \mathrm{~dB}$ at $850 \mathrm{MHz}$. This shows the large effect of fully-covered sheet metal. On the other hand, the shielding due to rebar structure is a function of the bar spacing relative to the wavelength $(\lambda)$, with greater shielding at lower frequencies (where the electrical spacing is $\leq \lambda / 10$ ). This phenomenon wasn't apparent in our measurements for two reasons: the spectral content of the short-pulse radiation was significantly higher than the lower-frequency range where this shielding would be effective; and the single-pass time-domain propagation was measured rather than the time-harmonic shielding effectiveness (which would include resonant scattering effects inside the walls and rebar structure).

At a much higher frequency (57 GHz), Sato et al. (1996) describes frequency-domain measurements of concrete in a free-space (radiated-transmission) setup. They examined the curing rate of concrete slabs over a period of 14 months, by utilizing both through and reflection 
measurements. Clemeña (1991) describes time-domain radar methods for characterizing concrete, with application to evaluating roadbed and bridge layer conditions, monitoring water hydration in concrete curing processes, etc. This illustrates one of the applications of ground-penetrating radar in commercial or industrial work.

Recent concrete experiments were reported by Su et al. (1996); they have used a capacitive low-frequency fixture, a coaxial transmission-line structure for $50 \mathrm{MHz}$ to $1 \mathrm{GHz}$, and a radiated-transmission setup over the $2-$ to $10-\mathrm{GHz}$ range. Their goal is non-destructive evaluation of Portland cement concrete, especially during the curing process. Typical values of $\varepsilon_{r}$ were 6-8 over this range. It should be noted that their time-domain radiated-transmission method utilized multiple bounces inside the concrete slabs, with de-embedding of the dielectric parameters versus frequency. Our time-domain procedure, on the other hand, utilizes a single-pass propagation pulse through the thicker concrete walls, without any multiple-bounce behavior captured. This is easier for thicker walls, because the double-bounce time (representing internal ringing of the wave between the two surfaces) is proportional to the layer thickness. This allows longer clear time for the single-pass waveforms to be measured.

Appropriate models for the lossy dielectric medium are critical in determining electromagnetic system performance, and for these earth media, the most common form are Debye relaxation models or variations thereof. See Nyfors and Vainikainen (1989), King and Smith (1981), Gans et al. (1991), and Barthel et al. (1990) for discussions of Debye material response. In terms of propagation behavior through Debye or lossy media, see Sherman and Oughstun (1981), Hechtman and Hsue (both 1989 and 1990), and Andrews et al. (1982). Debye-type behavior is classically shown by plots of the real and imaginary parts of the relative complex permittivity versus $\log$ frequency, with the real part ( $\varepsilon^{\prime}$, or our 'dielectric constant' $\varepsilon_{r}$ ) dropping through the relaxation frequency, and the imaginary part (our 'loss factor' $\varepsilon$ ") exhibiting a resonance absorption peak at the relaxation frequency. Hoekstra and Delaney (1974) showed this type of behavior for four different soils, especially for higher moisture content; the relaxation frequencies were in the 1-4 GHz range. It remains to be seen if our sand data or concrete-wall data exhibits this behavior; the limited project schedule prevented us from examining these models for our data, so this is a major item for future work. The sand measurements may show Debye-type behavior, but it was only slightly moist, so we don't expect strong polar water relaxation response. The concrete-wall measurements may show the same behavior as the sandbox, because the walls were fairly dry as well.

\section{Radiated Transmission Measurement Technique}

In terms of measuring the dielectric constant, $\varepsilon_{r}(f)$, and the effective loss tangent, $p_{e}(f)$, of lossy dielectric materials, a wide variety of techniques have been developed. Examples of measurement techniques are given by von Hippel (1954), Nyfors and Vainikainen (1989), King and Smith (1981), Miller (1986), Carpenter (1990), Bucci et al. (1972), Musil and Zacek (1986), Clemeña (1991), and Rose et al. (1972). These methods cover the gamut of frequency range ( $\mathrm{kHz}$ to $\mathrm{GHz}$ ) and dielectric properties (from very low loss to very lossy/conductive media). They can be classified as either frequency-domain or time-domain approaches, and either in-situ 
or sampled-material methods. There are well-known advantages and disadvantages for frequency-domain versus time-domain metrology, but because we are primarily developing time-domain electromagnetic system concepts, we have been more interested in the latter. Both approaches offer valuable information, and we should utilize whatever insights can be gained with each.

In terms of in-situ versus sampled-material methods, even though sampled methods (e.g., coaxial transmission-line cells or electromagnetic cavities) offer more controlled material conditions and better resulting accuracy, we prefer in-situ methods. There are several reasons: some system measurements physically dictate the setup; in-situ setups avoid the intrinsic error involved in sampling and controlling the material under test; and sampled setups often have frequency or bandwidth limitations due to their geometry and/or size. For example, to measure soils with sampled methods, the soil is removed from the earth, dried, sifted for uniformity, then moisture is added in careful laboratory procedures. This obviously removes the original variabilities in the in-situ soil conditions, but raises the question of what changes were introduced by the sampling process. In addition, transmission-line methods have an inherent upper-frequency limit due to waveguide or line overmoding; this causes problems when trying to measure, for example, concrete, which has relatively large gravel pieces inside.

As a result, this research effort has focused on free-space radiated transmission configurations which permit in-situ characterization of walls and other building or earth features. This method utilizes a radiated electromagnetic wave which is launched by a transmitting antenna, propagates through a material layer of some thickness, and is captured by a receiving system. References include Nyfors and Vainikainen (1989), Musil and Zacek (1986), Maurens et al. (1992), and Ghodgaonkar et al. (1989). It has also been used for millimeter-wave optoelectronics systems see Arjavalingam et al. (1990) as an example.

The free-space radiated transmission technique models the material layer under test as a two-port device, with an overall transfer function which relates the output pulse to the input excitation pulse. The other applicable free-space measurement technique is a reflection measurement, in which the backscattered electromagnetic wave off the front surface of a layer is captured. In this configuration, described by Nyfors and Vainikainen (1989), Musil and Zacek (1986), and Rose et al. (1972), the measured transfer function is a model of the reflection coefficient at the surface boundary in terms of the dielectric properties of the material. The radiated transmission method is ideal for measuring the attenuation of concrete layers (e.g. walls), and assembled layers of representative soil types. The radiated reflection method is ideal for ground-penetrating radar measurements, where backscattered waves are the only available means of probing or sensing the media of interest. The dielectric constant can be measured in a straightforward manner with either method, whereas the loss tangent is most accurately measured with the transmission method (it is difficult to measure loss tangent using surface backscatter data).

Because the radiated transmission configuration offers a measurement of both the attenuation and dispersion of representative material layers, and because the published methods weren't optimum for our requirements, a detailed measurement technique was developed specifically for 
this configuration. It is a direct time-domain approach which offers wideband spectral content in a straightforward procedure. By measuring two waveforms, one through a layer and the other a free-space reference pulse, a transfer function can be derived for the layer under test. This transfer function can then be used to unfold or determine the dielectric constant and loss tangent of the layer material.

The derivation of the short-pulse propagation measurement technique is as follows. Figure 1 depicts the radiated transmission problem, consisting of a short-duration electromagnetic excitation wave, $E_{i}(t)$, impinging on a homogeneous, isotropic material layer of thickness $d$, and a transmitted pulse, $E_{t 2}(t)$, emerging from the other side, which is then measured. The goal is to determine $\varepsilon_{r}(f)$ and $p_{e}(f)$ for the material layer. For simplicity we assume normal incidence (all wave propagation occurs normal or perpendicular to the planar boundaries) and that the incident pulse length is shorter than the transit time through the layer. By appropriate use of time-gating procedures, extraneous propagation paths are eliminated from the measurement; this includes multiple reflections within the layer, undesired reflections off the sides of finite-sized layers, antenna ringing, etc. As a result, this is a single-pass time-duration-limited transient measurement procedure which assumes a 1-D model of plane-wave propagation through a planar layer.

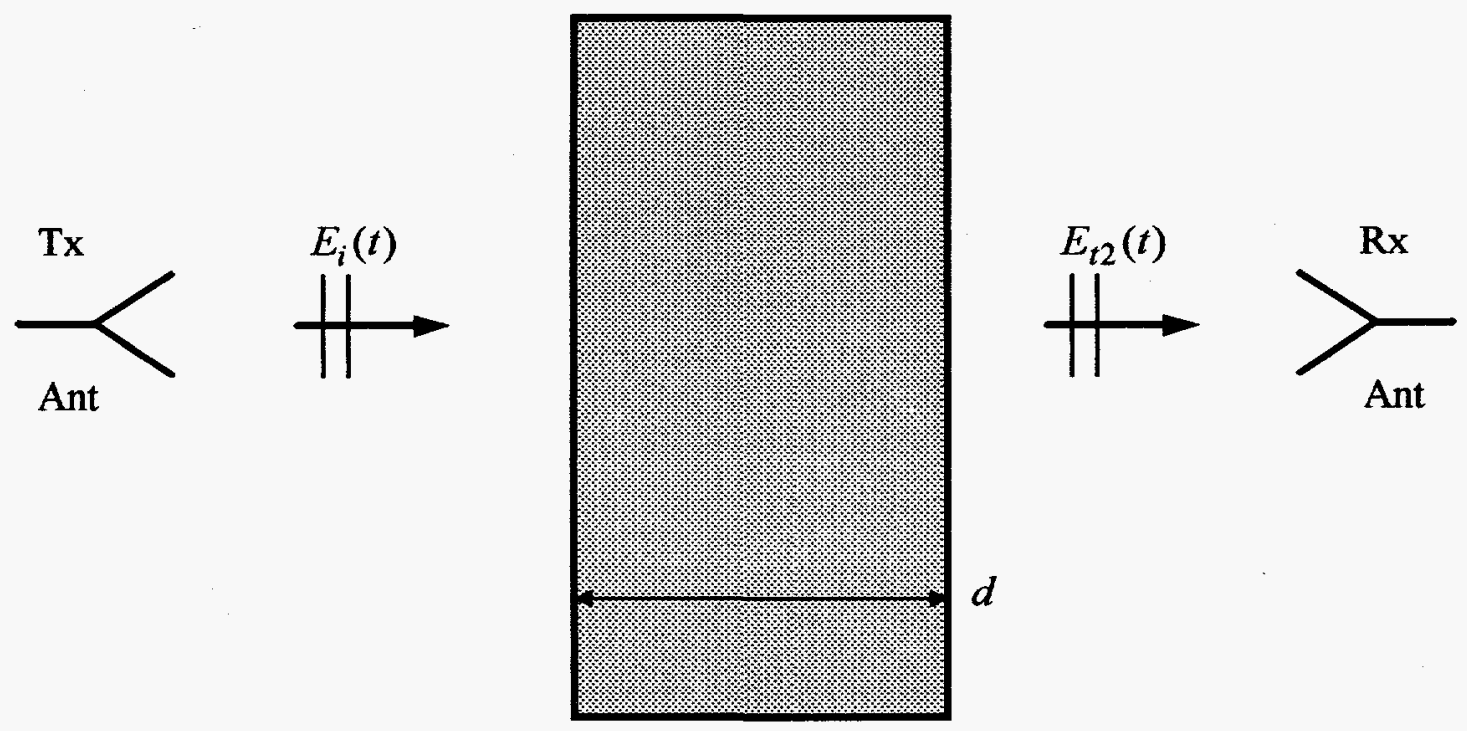

Material Layer, $\varepsilon_{r e}(f), \eta(f)$

Figure 1. Short-Pulse Plane-Wave Propagation Configuration.

In order to analyze the wave propagation at the front and rear boundaries of the layer, we construct a lattice or bounce diagram as in Figure 2 below. Two pulse measurements are made: - the transmitted 'through' pulse, $E_{t 2}(t)$, with the layer in place; and - a free-space reference pulse, $E_{t 2}^{f s}(t)$, which is the received wave without the layer. This reference pulse will be used to remove the non-ideal system response of the transmitting and receiving hardware. Care must be taken to ensure that the relative orientation of the transmitting and receiving antennas is kept as identical as possible for these two setups. 


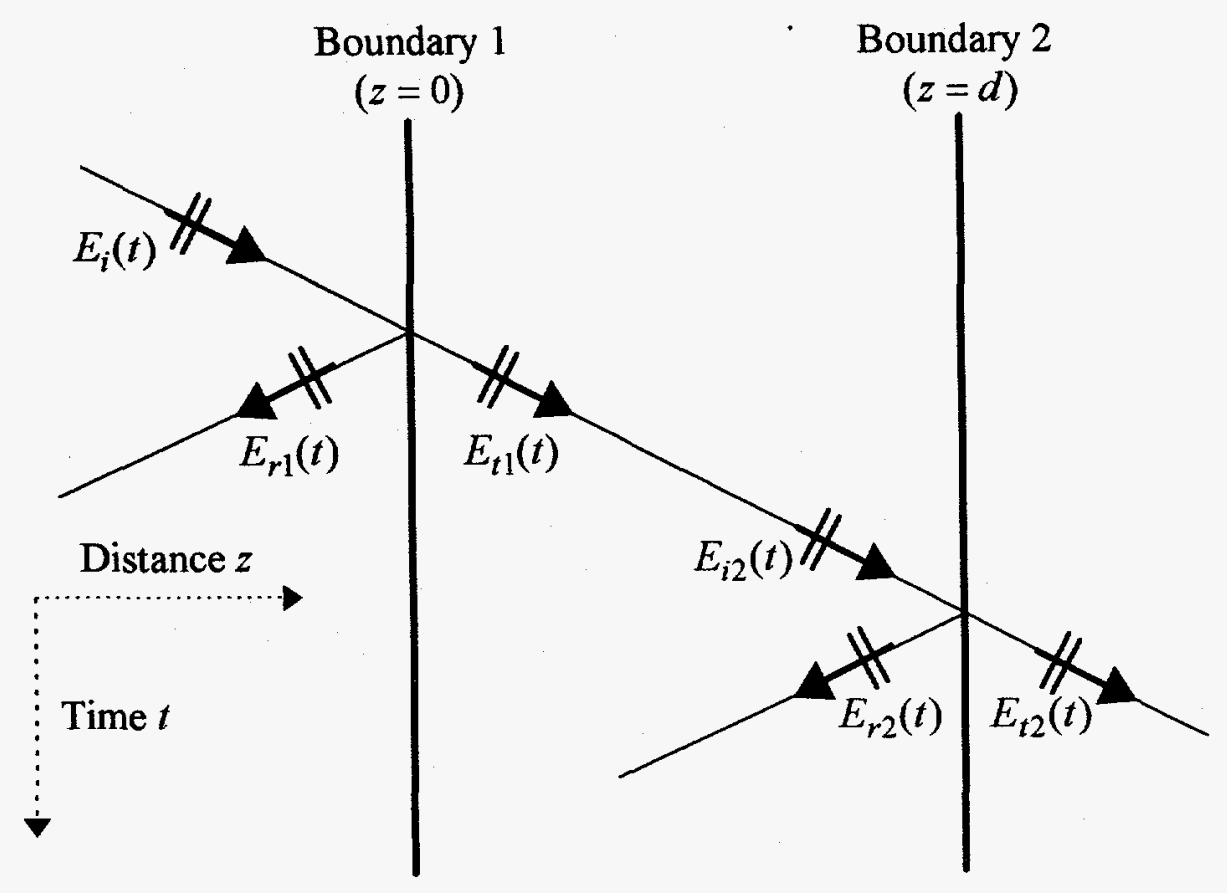

Figure 2. Bounce Diagram for Short-Pulse Propagation Measurements.

These two measured waveforms represent the electromagnetic wave incident on and picked up by the receive antenna. But note that we don't need to specify the transfer function of the receiving system (antenna and digitizer) in these measurements, because it drops out of the following spectral ratios. If the time-domain waveforms are acquired with the same receiver settings, and over a short enough time-frame for good instrument stability, then the receiver transfer function can be assumed to be identical for the two pulse acquisitions. However, if we needed to specify the incident electromagnetic wave on the layer (which is radiated by the transmitter), then we would have to characterize the receiver system and deconvolve its' transfer function out of the field measurement.

At the first boundary, the partial reflection coefficient is defined as the spectral ratio of the first reflected pulse, $E_{r 1}(t)$, and the incident pulse, $E_{i}(t)$ :

$$
\Gamma(f) \equiv \frac{E_{r 1}(f)}{E_{i}(f)} .
$$

These spectra are the discrete Fourier transforms of the respective waveforms. Note that this is single pass, not the sinusoidal steady-state case - it does not include the infinite number of internal reflections which would be present. Thus, this partial reflection coefficient is not the aggregate reflection coefficient which would be obtained in the usual time-harmonic solution of this layered problem. It does however correspond to the time-harmonic reflection coefficient for a lossy half-space problem, because there would be no internal standing-wave behavior as in the layered geometry. See Miller (1986), Pozar (1990), and Collin (1992) for further details about this formulation and the relationship between ringing time-domain phenomena and the resulting frequency-domain standing-wave behavior inside a transmission line or a propagation layer. 
This partial reflection coefficient can be couched in terms of the material properties of the layer as follows. For electromagnetic waves in lossy dielectric media, the complex wave impedance is given by Equation 5. And just like TEM transmission-line theory, the partial reflection coefficient is given by the following equation, based on the wave impedance in the layer and the free-space wave impedance (ahead of the boundary):

$$
\Gamma(f)=\frac{\eta(f)-\eta_{0}}{\eta(f)+\eta_{0}}=\frac{1-\sqrt{\varepsilon_{r e}(f)}}{1+\sqrt{\varepsilon_{r e}(f)}} .
$$

The wave transmitted across the first boundary, $E_{t 1}(t)$, is determined by the partial transmission coefficient across the boundary, $T_{1}(f) \equiv E_{t 1}(f) / E_{i}(f)$. By solving the boundary conditions for the electromagnetic fields, we have a general relationship between the reflection coeffficient and the transmission coefficient (note that both are complex): $T_{1}(f)=1+\Gamma(f)$. Then the wave propagates through the layer, and the pulse incident on the second boundary is $E_{i 2}(t)$, whose spectrum is given by $E_{i 2}(f)=E_{t 1}(f) e^{-\gamma(f) d}$. This is the time-harmonic exponential decay in bulk material as expressed in the complex propagation constant. Then the partial reflection coefficient at this rear boundary is defined to be $\Gamma_{2}(f) \equiv E_{r 2}(f) / E_{i 2}(f)=-\Gamma(f)$, and the corresponding transmission coefficient is

$$
T_{2}(f) \equiv E_{t 2}(f) / E_{i 2}(f)=1+\Gamma_{2}(f)=1-\Gamma(f) \text {. }
$$

The emerging pulse, $E_{t 2}(t)$, which is then picked up by the receiving antenna, has a spectrum of $E_{t 2}(f)=T_{2} E_{i 2}=T_{2}\left(E_{t 1} e^{-\gamma d}\right)=T_{2} T_{1} e^{-\gamma d} E_{i}(f)$. In terms of the first partial reflection coefficient, $\Gamma(f)$ in Equation 6, this yields a radiation transfer function of

$$
\frac{E_{t 2}(f)}{E_{i}(f)}=T_{2} T_{1} e^{-\gamma d}=(1-\Gamma)(1+\Gamma) e^{-\gamma d}=\left(1-\Gamma^{2}\right) e^{-\gamma d}
$$

After a measurement of $E_{t 2}(t)$ is made with the layer in place, then a measurement of the received wave is made without the layer. This is $E_{t 2}^{f s}(t)$, and it contains the non-ideal response of the pulser, the transmitting antenna, receiving antenna, and waveform recorder. The Fourier spectrum of the free-space measurement yields a corresponding radiation transfer function which merely represents a propagation delay across the imaginary layer of thickness $d$ :

$$
\frac{E_{i 2}^{f_{s}}(f)}{E_{i}(f)}=T_{2} T_{1} e^{-\gamma_{\theta} d}=(1)(1) e^{-j \omega d / c}=e^{-j \omega \tau_{0}},
$$

where $\tau_{0} \equiv d / c$ is the free-space transit time across the layer thickness. This is simply a linear phase shift due to the propagation delay; there is no interface loss at either boundary because the wave impedance doesn't change (the entire propagation region is that of free space).

Finally, we arrive at the desired characterization of the material layer - the insertion transfer function, defined as

$$
H(f)=\frac{E_{t 2}(f)}{E_{\imath 2}^{f}(f)}=\left(1-\Gamma^{2}\right) e^{-\left(\gamma-\gamma_{0}\right) d} .
$$


It is formed by the ratio of the two radiation transfer functions above (Equations 7 and 8):

$$
H(f) \equiv \frac{E_{t 2}(f) / E_{i}(f)}{E_{t 2}^{f_{s}}(f) / E_{i}(f)}=\frac{E_{t 2}(f)}{E_{t 2}^{f_{s}}(f)} .
$$

Notice that in concept this is similar to shielding effectiveness, because it represents the frequency-domain ratio of the emerging/transmitted wave amplitude reduction due to the presence of the layer under test. As such it contains the attenuation and dispersion of the layer versus frequency. In linear systems analysis this model is a two-port representation of a lossy, causal network, and in microwave metrology $H(f)$ is a measurement of the scattering parameter $S_{21}(f)$.

After substituting our expressions for the partial reflection coefficient, $\Gamma(f)$ of Equation 6 , and the complex propagation constant, $\gamma(f)$ of Equation 2, into the equation above, we obtain the layer transfer function in terms of the relative complex effective permittivity of Equation 1 , $\varepsilon_{r e}(f)$ :

$$
H(f)=\frac{4 \sqrt{\varepsilon_{r e}}}{\left(1+\sqrt{\varepsilon_{r e}}\right)^{2}} e^{-j \omega \tau_{0}\left(\sqrt{\varepsilon_{r e}}-1\right)} .
$$

Once this complex transfer function is obtained from the ratio of spectra of the two received waveforms, then the complex permittivity is obtained by a transcendental solution of this equation. The result will be an estimate of the dielectric constant and effective loss tangent at each frequency of the discrete Fourier spectra. Note that fast Fourier transforms (FFTs) are used for the numerical processing.

In summary, the new radiated transmission measurement procedure is as follows. First, we measure the 'through' pulse, $E_{t 2}(t)$, with the material layer in place. Second, we measure the free-space reference pulse, $E_{t 2}^{f_{s}}(t)$, with the layer removed. This was accomplished in two different ways for our propagation experiments. In Series 1, we designed and built a rigid antenna platform for holding the transmit and receive antennas in a fixed orientation, which we then physically moved over a finite-sized slab or layer under test. This permitted very precise comparison of the two propagation conditions. In Series 2 we had to be able to measure the walls of buildings in-situ, which prevented the use of such an antenna platform. Instead we had to set up two physically different radiated-transmission configurations, one with the transmitter on one side of a wall and the receiver on the other side, and the other a free-space configuration. In this case the antenna orientations were very carefully measured and aligned in order to minimize the errors introduced by differing antenna radiation conditions.

The third step in this short-pulse propagation technique is to perform signal processing; we compute the transfer function of the layer, and then unfold the dielectric constant and loss tangent from the transfer function. In addition to this frequency-domain formulation, we can compute the time-domain impulse response of the layer, which is given by the inverse Fourier transform of the transfer function. We can also form the time-domain cross-correlation of the two measured waveforms, and determine the propagation delay due to the layer characteristics. Another 
time-domain quantity can be computed as well: the total energy in the two waveforms - their ratio represents the attenuation of the short-duration pulses through the layer.

\section{Low-Loss Analysis Method}

Using the time-domain measurement procedure described above, we obtain a frequency-domain complex transfer function, $H(f)$, representing the material layer under test. Then the dielectric constant and effective loss tangent may be computed from each spectral component of $H(f)$, assumed to be given by Equations 9a and/or 9c. Unfortunately, this requires an iterative numerical technique, because the solution for these complex permittivity parameters is transcendental and implicit. Because such iterative algorithms are difficult to perform with our data analysis software (DADiSP, by DSP Development Corp.), we derived an alternative analysis method which is explicit and thus much easier to implement. The transcendental inversion of the transfer-function equation above may be simplified by making an assumption that the material is low loss.

This results in two separate simplifications. The first simplification of Equation $9 \mathrm{a} / 9 \mathrm{c}$ is that the mismatch transmission losses at the two boundaries are approximately real-valued. That is, if the loss tangent $p_{e}(f) \ll 1$, then it may be dropped out of the square roots:

$$
T_{2} T_{1}=\left(1-\Gamma^{2}\right)=\frac{4 \sqrt{\varepsilon_{r e}(f)}}{\left[1+\sqrt{\varepsilon_{r e}(f)}\right]^{2}}=\frac{4 \sqrt{\varepsilon_{r}\left(1-j p_{e}\right)}}{\left[1+\sqrt{\varepsilon_{r}\left(1-j p_{e}\right)}\right]^{2}} \cong \frac{4 \sqrt{\varepsilon_{r}}}{\left(1+\sqrt{\varepsilon_{r}}\right)^{2}} .
$$

The resulting quantity is real-valued rather than complex. As an example of the error introduced by this approximation, assume poor conditions for this approximation (very lossy, but lower dielectric constant than normal for such loss): $\varepsilon_{r}=10$ and $p_{e}=0.2$. The approximate value for $T_{2} T_{1}$ is 0.7301 , which is only $0.3 \%$ greater than the magnitude of the exact value, $T_{2} T_{1}=0.7277 \angle 3^{\circ}$. The corresponding phase error introduced by making this real-valued is only $3^{\circ}$. This is better accuracy than the time-domain hardware can provide for the raw waveform measurements, so it is very acceptable.

The second simplification is that the complex exponential portion of Equation $9 \mathrm{a} / 9 \mathrm{c}$,

$$
e^{-\left(\gamma-\gamma_{0}\right) d}=e^{-\left(\alpha+j \beta-j \beta_{0}\right) d}=e^{-j \omega \tau_{0}\left[\sqrt{\varepsilon_{r e}(f)}-1\right]},
$$

may be separated into independent magnitude and phase terms. This is done by examining the attenuation and phase constants defined earlier. Here $\tau_{0} \equiv d / c$ is the free-space transit time across the layer thickness. In both terms we replace the expression $\sqrt{1+p_{e}^{2}}$ with the Taylor-series expansion for small loss tangent: $\sqrt{1+p_{e}^{2}} \approx 1+\frac{1}{2} p_{e}^{2}-\frac{1}{8} p_{e}^{4}+\ldots$. For the attenuation constant (Equation 3 ) only the first two terms are retained, yielding

$$
\alpha(f)=\frac{\omega}{c}\left\{\frac{\varepsilon_{r}}{2}\left[\sqrt{1+p_{e}^{2}}-1\right]\right\}^{1 / 2} \cong \frac{\omega \sqrt{\varepsilon_{r}} p_{e}}{2 c} .
$$

For the phase constant (Equation 4 ) only the first term is kept, resulting in 


$$
\beta(f)=\frac{\omega}{c}\left\{\frac{\varepsilon_{r}}{2}\left[\sqrt{1+p_{e}^{2}}+1\right]\right\}^{1 / 2} \cong \frac{\omega \sqrt{\varepsilon_{\mathrm{r}}}}{c} .
$$

These constants are then re-inserted into the exponential term of the transfer function:

$$
e^{-\left(\alpha+j \beta-j \beta_{0}\right) d} \cong e^{-\alpha(f) d} e^{-j \omega \tau_{0}\left[\sqrt{\varepsilon_{r}(f)}-1\right]} .
$$

Combining the results of both simplifications, we arrive at the low-loss version of the layer transfer function:

$$
H(f) \cong \frac{4 \sqrt{\varepsilon_{r}}}{\left(1+\sqrt{\varepsilon_{r}}\right)^{2}} e^{-\alpha d} e^{-j \omega \tau_{0}\left(\sqrt{\varepsilon_{r}}-1\right)} .
$$

This version of the transfer function has separable magnitude and phase components shown below:

$$
H(f)=|H(f)| e^{j \Phi(f)} \cong\left[\frac{4 \sqrt{\varepsilon_{r}}}{\left(1+\sqrt{\varepsilon_{r}}\right)^{2}} e^{-\frac{1}{2} \omega \tau_{0} \sqrt{\varepsilon_{r}} p_{e}}\right]\left[e^{-j \omega \tau_{0}\left(\sqrt{\varepsilon_{r}}-1\right)}\right],
$$

where $\Phi(f)$ is the phase component (given by the second term). As an example of the error introduced in $|H(f)|$, consider the generic poor scenario given above, with parameters of $\varepsilon_{r}=10, p_{e}=0.2$, and $f=1 / \tau_{0}$ (like $\tau_{0}=1 \mathrm{~ns}$, and $f=1 \mathrm{GHz}$ ). The approximate formula yields a magnitude of 0.7230 compared to the exact value of 0.7277 ; this is an error of only $-0.6 \%$, which is quite adequate.

Using the low-loss formulation derived above, the two-step procedure for processing the measured transfer function is then as follows. The first step is to determine the dielectric constant from the slope of the phase shift, using the differential time delay, $\Delta \tau$, introduced by the layer: $\Delta \tau(f) \equiv \tau_{0}\left[\sqrt{\varepsilon_{r}(f)}-1\right]$. The phase shift versus frequency is $\Phi(f) \cong-2 \pi f \Delta \tau(f)$, and the derivative is $\frac{d \Phi}{d f} \cong-2 \pi \Delta \tau(f)$, so that the differential time delay is given by $\Delta \tau(f) \cong-\frac{1}{2 \pi} \frac{d \Phi(f)}{d f}$. Solving for the dielectric constant, we have

$$
\varepsilon_{r}(f) \cong\left[1+\frac{\Delta \tau(f)}{\tau_{0}}\right]^{2}=\left[1-\frac{1}{2 \pi \tau_{0}} \frac{d \Phi(f)}{d f}\right]^{2}
$$

The second processing step is to determine the loss tangent of the layer from the magnitude of the transfer function (in Equation 10b) and the just-computed dielectric constant (11):

$$
p_{e}(f) \cong-\frac{1}{\pi f \tau_{0} \sqrt{\varepsilon_{r}(f)}} \ln \left[\frac{\left[1+\sqrt{\varepsilon_{r}(f)}\right]^{2}}{4 \sqrt{\varepsilon_{r}(f)}}|H(f)|\right] \text {. }
$$

This simplified analysis method for determining the complex permittivity parameters from the measured layer transfer function has worked well for the media we have characterized, at least over the parameter ranges encountered so far: dielectric constants in the range of 2.5-8; and loss tangents $<0.2$. 


\section{Series-1 Experiments}

After we developed the radiated transmission measurement technique (described in the preceding section), we performed two series of transient propagation experiments. The Series-1 experiments were meant to be a short-term verification of the measurement technique. Even though the substantial data for this report were obtained in the Series-2 experiments, it is nevertheless worthwhile to describe the Series-1 work as preparatory background for the improved measurements accomplished during the Series- 2 work. The Series- 1 experiments included the following materials:

- nylon slabs;

- temporary stacked walls of solid concrete cinder-blocks;

- an outdoor reinforced poured-concrete earth-berm wall; and

- an interior reinforced poured-concrete wall.

The experimental hardware preparation and nylon-slab validation experiments were done in late FY94, with the main propagation experiments performed in early FY95.

Two simple TEM horn antennas were assembled for the Series-1 measurements, with an emphasis on well-behaved time-domain impulse response and a clear-time length of $4 \mathrm{~ns}$. Their nominal surge impedance throughout was $50 \Omega$. The transmitting antenna was $63.5-\mathrm{cm}$ long, with an aperture width of $19.4 \mathrm{~cm}$ and aperture height (plate separation) of $3.6 \mathrm{~cm}$; the receiving antenna was $60.3-\mathrm{cm}$ long, $22.0-\mathrm{cm}$ wide, and $3.6-\mathrm{cm}$ high. The pulser was a Picosecond Pulse Labs $4015 \mathrm{~B}$ step generator with specifications of $-9 \mathrm{~V}$ amplitude and 10-90\% transition time of 15 ps. The waveform recorder was a Tektronix CSA-803 sampling oscilloscope, with an SD-26 sampling head (upper $-3 \mathrm{~dB}$ frequency specification of $20 \mathrm{GHz}$ ). The resulting free-space impulse waveform had a $50 \%$ pulse duration of $60 \mathrm{ps}$, with spectral content of $1-18 \mathrm{GHz}$.

In order to validate the time-domain measurement technique and low-loss analysis method, we assembled a double-slab layer of nylon as the test layer. The overall dimensions of this layer were a thickness $d=11.0 \mathrm{~cm}$, and a height and width of $1.23 \mathrm{~m}$. Figure 3 is a photograph of the experimental setup, showing a lightweight rigid plywood frame for the antennas. For the nylon measurements, the spacing between the antenna apertures was $81.4 \mathrm{~cm}$, and the height of the antenna centerlines was $58 \mathrm{~cm}$ above the concrete lab floor. The antenna platform was positioned so that the propagation centerline was centered in the width and height of the layer, in order to maximize the clear time for propagated pulses passing through the layer (before the arrival of lower-frequency diffracted waves around the nylon layer). We designed this wood frame to provide a fixed platform for the transmit and receive antennas during movement over a wall or test layer. The better the relative antenna position is maintained between the 'through' and the free-space measurements, the better the resulting accuracy of the measurement technique.

The time-domain impulse waveforms for the 'through' and the free-space configurations showed only slight pulse attenuation ( $12 \%$ reduction in peak values), and relatively little dispersion (the waveforms were very similar in shape). The peak-to-peak time delay of $251 \mathrm{ps}$ was exactly the same as the differential time delay predicted from a published value of the 
dielectric constant of nylon $(2.84$ at $3 \mathrm{GHz}): \Delta \tau \equiv \tau_{0}\left[\sqrt{\varepsilon_{r}}-1\right]=\frac{d}{c}\left[\sqrt{\varepsilon_{r}}-1\right]=251 \mathrm{ps}$.

Conversely, the derived dielectric constant versus frequency was shown to be very consistent with the published data for nylon. Using the low-loss analysis method described in the preceding section of this report, the mean value of $\varepsilon_{r}$ was 2.83 for the $3-16 \mathrm{GHz}$ range, and the loss tangent, $p_{e}$, was $0.006-0.011$ with a mean value of 0.0081 . This is excellent agreement with the published data for nylon material, and at least for very low-loss media it demonstrated the validity of our experimental approach. This time-domain approach was also sensitive enough to see the pulse formed by the double bounce inside each of the two nylon slabs; this was caused by the small vertically-oriented air gap in between the slabs.

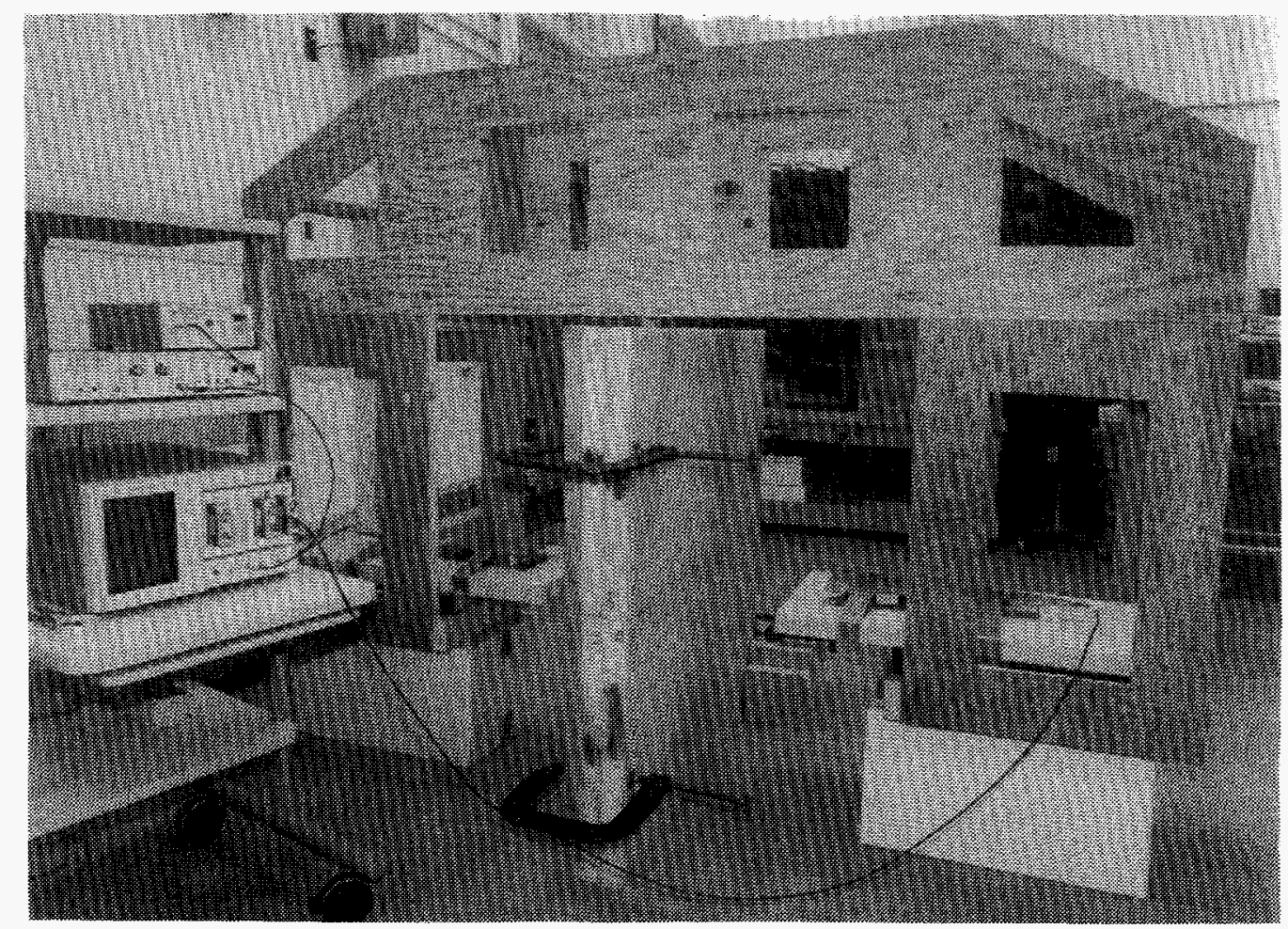

Figure 3. Experimental Configuration for Nylon-Slab Validation Measurements.

\section{Temporary Concrete-Block Walls}

After the nylon-slab validation measurements were completed, we then proceeded to perform two sets of propagation experiments - one set of temporary stacked walls of solid cinder blocks, and one set of permanent poured-concrete walls. The goals of the short-pulse measurements through temporary stacked walls were to examine the repeatability of our experimental technique and the propagation attenuation scaling with increasing thickness. We constructed three different walls, a wall of just one vertical layer of tightly-stacked blocks, a wall with two side-by-side vertical layers, and the last wall with three vertical layers. As an example, Figure 4 is a photograph of the experimental setup for the double-layer wall configuration. The commercial concrete blocks were solid with pumice aggregate, having a nominal weight of $6.58 \mathrm{~kg}$ each and a nominal density of $1.5 \mathrm{~g} / \mathrm{cm}^{3}$. The blocks were slightly damp, and were previously laid out on the 
lab floor (on end, for maximum air exposure) to stabilize at room temperature for uniform moisture content. These wall configurations were carefully assembled to be as tight as possible in order to minimize the wave propagation perturbations caused by air gaps between the blocks. Our radiated transmission method/model assumes plane-wave propagation conditions, so we attempted to construct smooth planar front and rear surfaces as well.

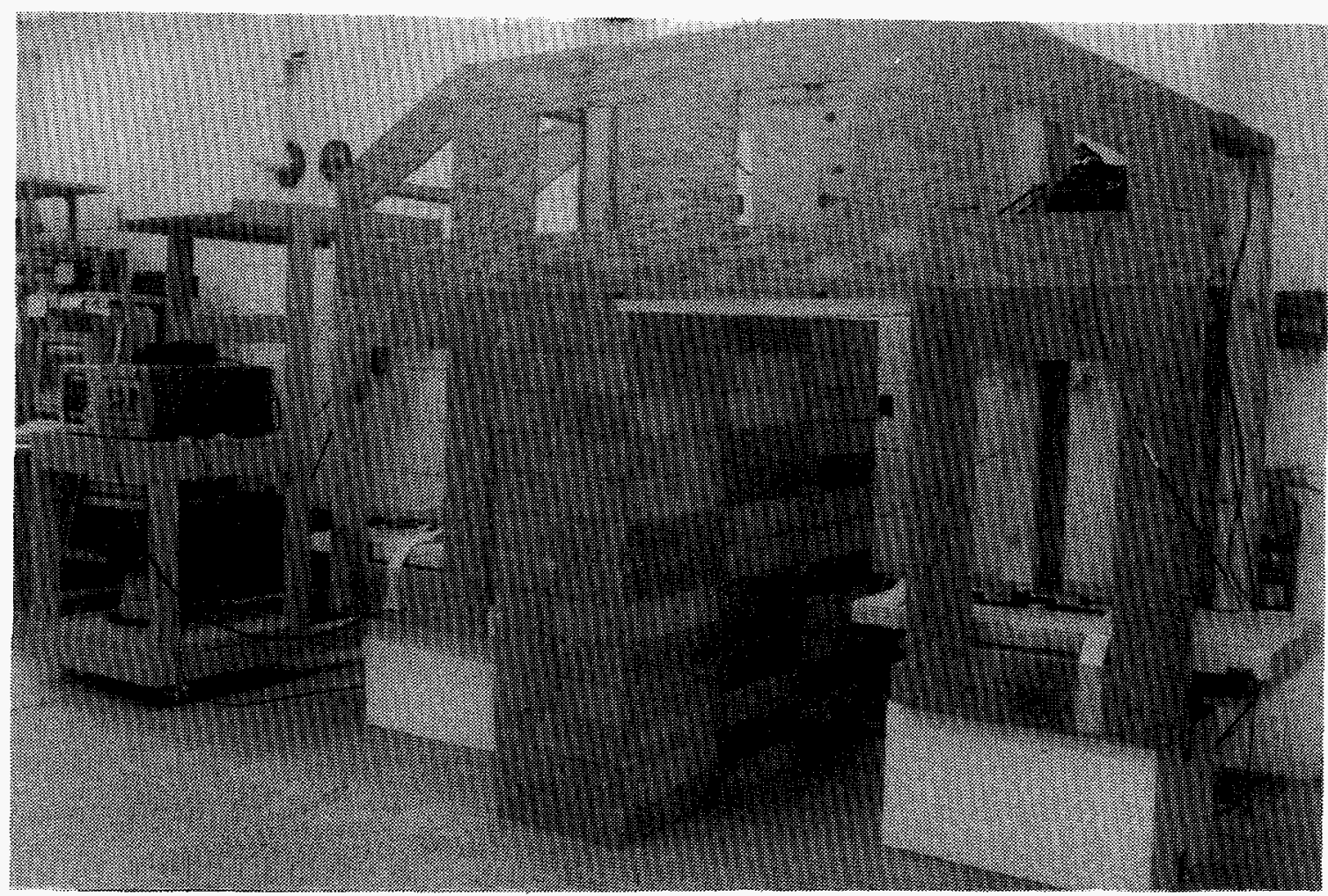

Figure 4. Experimental Configuration for Double-Layer Concrete-Block Wall.

Each of the block walls was $1.19 \mathrm{~m}$ wide by $1.21 \mathrm{~m}$ high, sitting on the lab floor. The antenna structure was positioned so that the antenna centerline was in the middle of these walls $(58 \mathrm{~cm}$ above the floor), and the aperture separation between the antennas was fixed at $1.00 \mathrm{~m}$. We attempted to make the walls as large as possible, but even this size yielded significant edge diffraction effects for the double- and triple-layer walls (where the direct 'through' wave experiences more time delay). In terms of the distance of each antenna to the wall surface, a compromise must be made - the spherical-wave spreading error is reduced by increasing the wall-antenna separation, but the error due to low-frequency diffracted energy around the wall edges increases with this separation. In addition, the received signal level drops in a $1 / r$ fashion as each antenna is pulled away from the wall or layer under test. This diffraction-path problem dictates that the wall size must be several times larger than the desired clear-time duration in the time-domain measurement, as pointed out by others who have implemented radiated transmission systems for dielectric material characterization. This issue of wall size is so important (for both time-domain and frequency-domain techniques) that it is often the limiting factor in measurement quality for radiated transmission propagation studies. This will be seen again (even more dramatically) in the Wright Lab sandbox data, to be described in the Series-2 experiments. 
The single-layer block wall had a thickness of $d=19 \mathrm{~cm}$, and a peak-peak impulse time delay of $\Delta \tau=1.085 \mathrm{~ns}$. If we use the time-domain peak-peak delay to determine a single representative value of dielectric constant rather than the general frequency-dependent version of Equation 11, then

$$
\varepsilon_{r} \cong\left[1+\frac{\Delta \tau}{\tau_{0}}\right]^{2}=\left[1+\frac{\Delta \tau}{d / c}\right]^{2} ;
$$

this implies that we are assuming minimal dispersion through the layer, so that the phase shift of the transfer function is primarily linear (and the differential time delay is flat or constant over the spectral bandwidth of the measurement). This was indeed the case for all of the data we collected in both Series- 1 and Series-2 experiments. The single-layer wall peak-peak delay thus yields a nominal dielectric constant of 7.34 based on this differential delay. Using this single value of time delay, the effective loss tangent had a mean value of 0.1 over the $1-10 \mathrm{GHz}$ range. In addition, the wall barely had sufficient clear time before edge diffraction effects were observed.

The double-layer block wall had a thickness of $d=38.5 \mathrm{~cm}$, and a peak-peak impulse time delay of $\Delta \tau=2.05 \mathrm{~ns}$, yielding a nominal dielectric constant of 6.75 with Equation 13. There was more attenuation (as expected due to twice the thickness), so our effective spectral content dropped to the $1-5 \mathrm{GHz}$ range. There were also definite edge diffraction effects, so we installed aluminum-foil shielding around the block wall in order to reduce the diffraction around the outside of the wall. Using the peak-peak time delay rather than that derived from the slope of the layer transfer function, the effective loss tangent was $0.1-0.2$ over the $1-5 \mathrm{GHz}$ range.

The triple-layer block wall had a thickness of $d=57.7 \mathrm{~cm}$, and a peak-peak impulse time delay of $\Delta \tau=3.09 \mathrm{~ns}$, yielding a nominal dielectric constant of 6.79. Again, there was even greater pulse attenuation due to the increased thickness, and as a result we had to incorporate a very wideband low-noise amplifier for the 'through' measurement. There was also definite edge diffraction but we didn't have time to incorporate the aluminum-foil shielding. The effective loss tangent was just like that of the double-layer wall, $0.1-0.2$ over a $1-7 \mathrm{GHz}$ range.

As a result, the three different concrete-block walls yielded fairly consistent phenomenology, with similar values of pulse delay (per layer thickness), dielectric constant, and effective loss tangent. This motivated us to continue using these specific measurement techniques.

\section{Permanent Concrete Walls}

Two permanent poured-concrete reinforced walls were characterized in the Series-1 experiments. Both are located at Building 6640, in Tech Area 3 at Sandia/New Mexico. One was an outdoor earth-berm wall, with the earth recently dug out in a region adjacent to the wall (to permit access to that face of the wall). The top of the earth-berm wall had a $30^{\circ}$ downward slope, following the shape of the earth berm, and the wood antenna platform was carefully placed over a section of the wall (tipped at the wall angle as well). The internal rebar was vertically oriented, so the electromagnetic wave polarization was slant linear ( $36^{\circ}$ off vertical). 
The earth-berm wall was $35.5 \mathrm{~cm}$ thick and slightly wet on the earth side (the dirt had been removed 7 days earlier, and the wall was visibly damp where it had originally been in contact with the soil). The time-domain measurements showed diffraction over the top edge of the wall, but not significant during the reception of the direct 'through' pulse. The impulse waveforms showed a peak-peak time delay of $1.98 \mathrm{~ns}$, which implies a nominal dielectric constant of 7.15 with Equation 13. This was consistent with the temporary-wall cinder-block data above. The insertion loss (magnitude of the transfer function, $|H(f)|)$ was large and comparable to the double- and triple-layer block walls. Analysis of the transfer function with Equations 10, 11, and 12 yielded a loss tangent of $0.05-0.18$ for the $500 \mathrm{MHz}$ to $4 \mathrm{GHz}$ frequency range.

The other permanent wall we measured (in both Series-1 and Series-2 experiments) was an interior wall in Building 6640 , the dividing wall between the antenna bay and rear high bay rooms. It is poured concrete construction, with three layers of vertical and horizontal rebar inside; we used a commercial rebar-locating sensor in an attempt to identify this rebar content, but it was too difficult to clearly locate the individual reinforcing steel bars due to their depth inside the wall. The transmit antenna was located on the east side of the wall, radiating through the wall to the receive antenna which was located on the west side of the wall. The first (eastern-most) rebar layer is $70 \mathrm{~mm}$ deep (below the front surface), and has vertical rebar spaced every $30 \mathrm{~cm}$ horizontally. The second (middle) layer has at least horizontal rebar, and the third rebar layer has both vertical and horizontal members at an average depth of $77 \mathrm{~mm}$ below the west surface of the wall. In this last layer the vertical members nearest the propagation path have a horizontal spacing of $37 \mathrm{~cm}$, and they are offset horizontally from the vertical members in the first rebar layer.

Both transmit and receive antennas were aligned for vertical linear polarization for these measurements and the antenna centerline position was varied over a $60-\mathrm{cm}$ square area, with real-time monitoring of the 'through' signal; there were variations as expected but no significant identifiable changes relative to the known rebar locations. Because the antenna polarization was vertical, we expected the dominant rebar scattering to be from vertical members rather than horizontal. For this measurement data the propagation centerline was $1.65 \mathrm{~m}$ above the floor (on each side of the wall), about $8 \mathrm{~cm}$ from a vertical rebar member in the first layer, and centered in-between two vertical members in the last layer. There was also a horizontal member in the last/third layer at about the height of the antenna/propagation centerline ray path. The blueprints of the building don't offer any additional details of the rebar construction.

Figure 5 shows the transmitting hardware for this internal wall experiment; the antenna is sitting on top of a wood and foam dielectric support stand, and the pulse generator is on a shelf below. Figure 6 shows the receiving hardware on the west side of the wall, with the antenna and Tektronix SD-26 sampling head on the top of the platform, and the sampling oscilloscope mainframe below. The antenna aperture-to-wall separation was $30 \mathrm{~cm}$ on each side. 


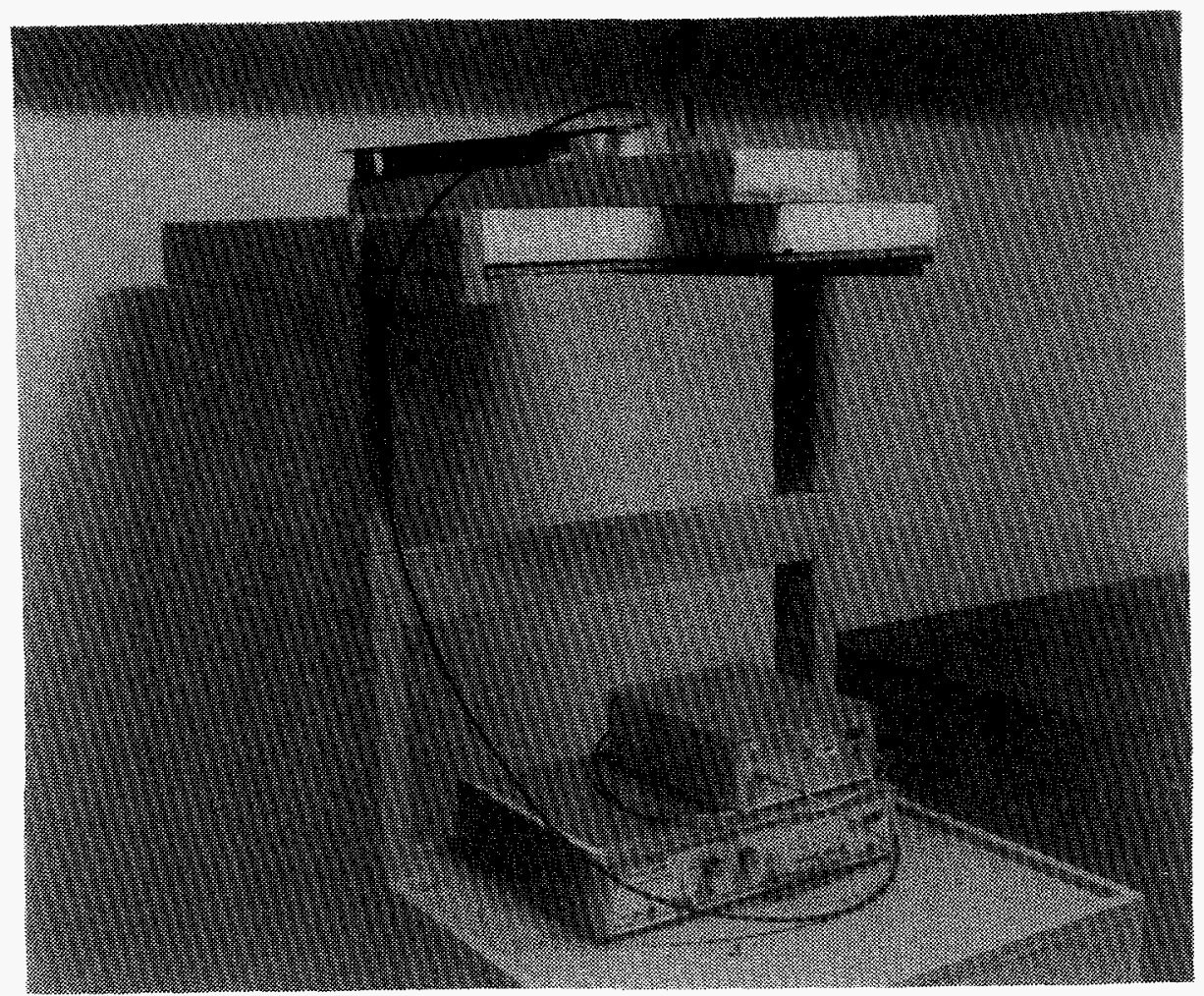

Figure 5. Transmitting Hardware for Internal Sandia Concrete Wall.

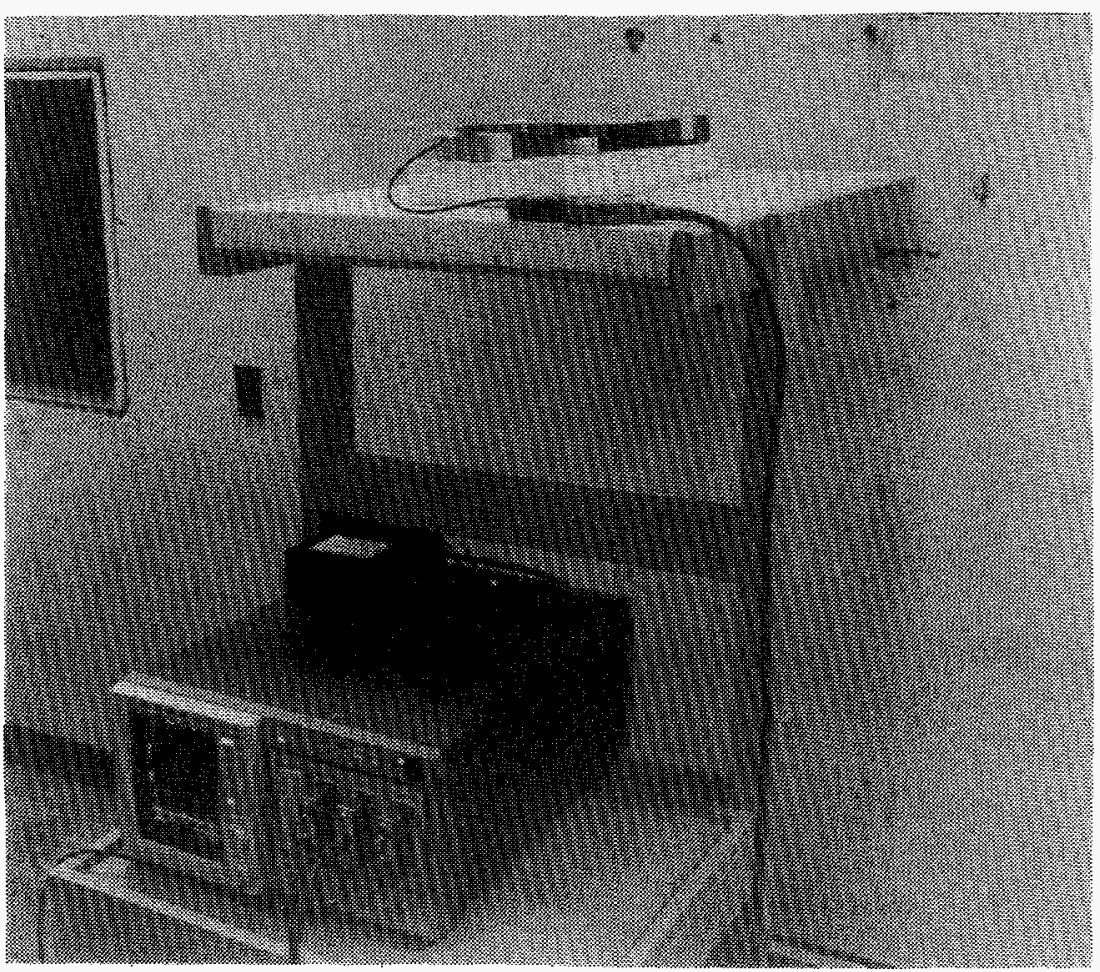

Figure 6. Receiving Hardware for Internal Sandia Concrete Wall. 
The interior wall, with a nominal thickness of $d=30.5 \mathrm{~cm}$, had a peak-peak impulse time delay of $\Delta \tau=1.27 \mathrm{~ns}$, yielding a nominal dielectric constant of 5.06. Unlike the other wall measurements which had significant diffracted energy around or over the finite-sized structures, this full wall offered complete shielding and no edge diffraction effects; the data was much cleaner. This wall had lower attenuation and dielectric constant than the other wall configurations, with a loss tangent of $0.02-0.05$ over the $1-14 \mathrm{GHz}$ band. It definitely had lower moisture content than the temporary cinder-block walls or the outside earth-berm wall. 
Blank Page 


\section{Series-2 Experiments}

After the Series-1 experiments were completed, we upgraded the time-domain measurement hardware, including the design and construction of a pair of new TEM horn antennas. Then we performed the Series-2 propagation experiments with the goal of carefully characterizing the transient propagation through concrete and sand. This included work at two facilities, Sandia/New Mexico, and USAF Wright Laboratory Armament Directorate (Eglin AFB, FL). Dr. Kwang Min was the point of contact at Wright Lab, in the Fuzes Branch. The materials tested during the Series-2 experiments were:

- the same interior concrete wall as that used in the Series-1 work (at Sandia Labs);

- another interior concrete wall (at Wright Lab); and

- a sandbox (6 ft. thick, also at Wright Lab).

The Sandia wall is described in the Series-1 section of this report. The Wright wall, like the Sandia wall, is also permanent (part of a building), of poured-concrete construction, with three internal layers of rebar, and having a nominal thickness of 12 in. Because of their strong similarity, these two walls offered a good comparison of the propagation phenomenology. The sandbox was constructed at Wright Lab for this type of measurement characterization, and is $1.9 \mathrm{~m}$ thick, $3.6 \mathrm{~m}$ wide, and $2 \mathrm{~m}$ high, with pressure-treated wood walls and filled with the typical fine, white sand of that region in Florida.

The wideband time-domain measurement system consisted of similar instrumentation as employed in the Series-1 work, but with several upgrades. The same Picosecond Pulse Labs (PSPL) 4015B pulse generator was used for the transmitter, but with optimized cabling for maximum radiated field and fastest transient performance. The receiver incorporated the same Tektronix CSA-803 sampling oscilloscope, but with a 50-GHz SD-32 sampling head (graciously loaned to us by Tektronix) rather than the $20-\mathrm{GHz}$ SD-26 head used before. In addition, after the Series-1 measurements we determined that much better antennas were necessary for the main experimental thrust of the Series- 2 work.

We have been involved in time-domain antenna development since 1990 [e.g., Clark et al. (1993) and Aurand (1994)], with considerable initial assistance from Dr. Arthur Ondrejka at the National Institute of Standards and Technology [Lawton and Ondrejka (1978) and Ondrejka et al.]. Consequently, we had several ideas in mind regarding our next generation of TEM horn antennas. So considerable effort was spent in designing and building an identical pair of new antennas, as well as optimizing the entire time-domain measurement system to have the fastest transient response possible (thus offering the widest bandwidth of wall characterization). See the Appendix for complete details about the new antenna, a TEM horn with dielectric aperture lens. It was specifically designed to have the fastest possible main-beam transient response. We used the solid-plate configuration of the new TEM horn for these Series-2 measurements rather than the printed-board configuration because of the much faster transient response of the solid-plate version. 
A variety of time-domain pulse types were used in these measurements in order to determine their effect on the transient propagation-loss behavior through the media we studied. The TEM horn antennas we built and utilized for these Series- 2 measurements have a clean derivative transmitting time-domain response (on boresight), so the radiated electromagnetic field is a derivative of the pulse which drives the transmit antenna. The receiving response of an antenna is a time integral of the transmitting response, so upon reception this antenna produces an output voltage pulse which is a replica of the incident wave. As a result, with step excitation of the transmit antenna, the radiated wave incident on the wall under test is an impulse, and the system has a free-space received pulse which is also an impulse. For the Sandia wall experiments, four impulse (or unipolar) waves were created with different pulse durations. For the Wright wall experiments, five different impulse waves were employed, as well as a bipolar or single-cycle sinusoidal type of pulse waveform. For the sandbox experiments, a single impulse waveform was used.

\section{Sandia Labs Concrete Wall}

The experimental setup at our facility consisted of the same overall configuration as shown in Figures 5 and 6, with the new antennas rather than the Series-1 antennas. For this 'through' measurement, the distance between each antenna aperture and the wall was $30.0 \mathrm{~cm}$, for a total separation of the antennas of $90.5 \mathrm{~cm}$ (the nominal wall thickness is 12 in. or $30.5 \mathrm{~cm}$ ). Figure 7 is a block diagram of the instrumentation hardware which we used for this time-domain experiment.

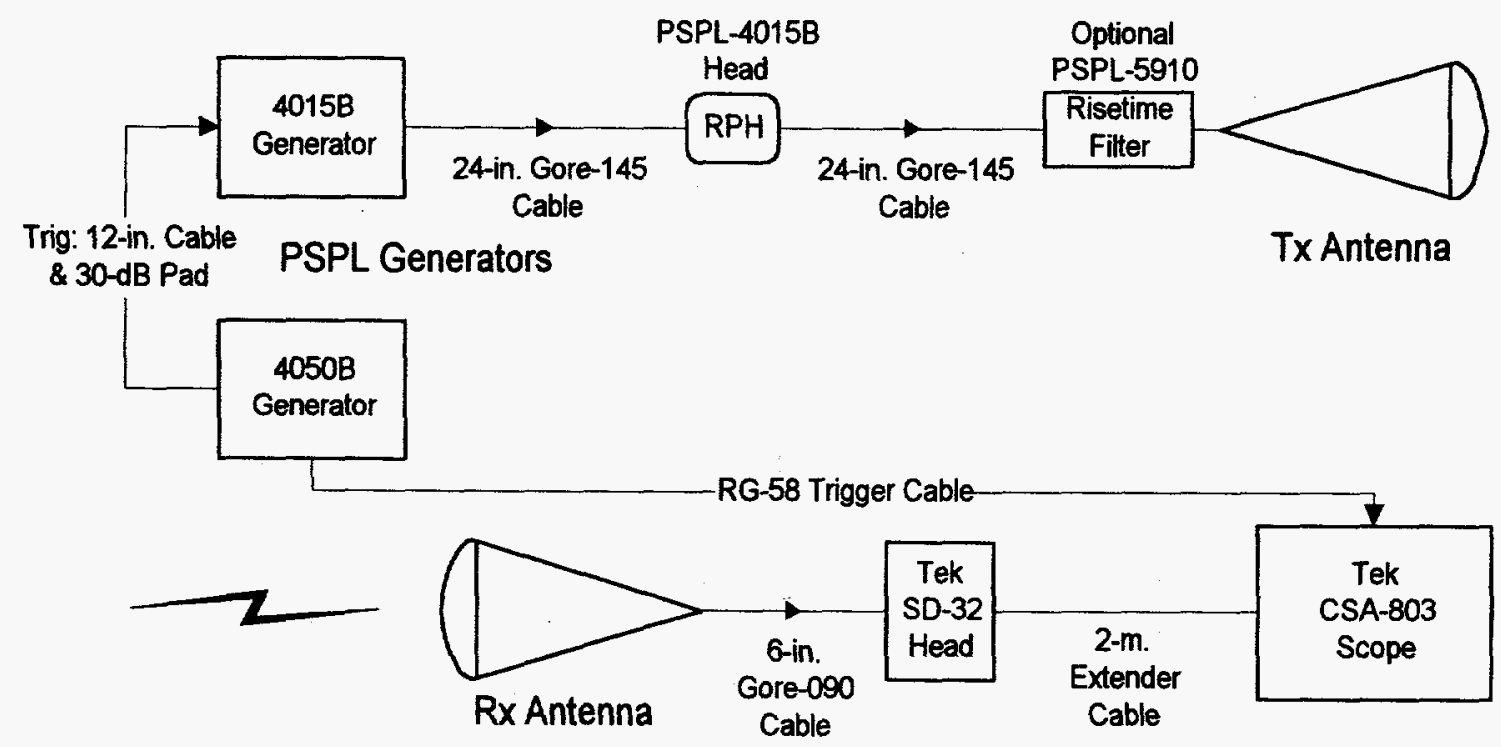

Figure 7. Block Diagram of 'Through' Setup for Sandia Concrete Wall.

One of our PSPL 4015B generators was modified to have a pulse length of 5 ns rather than the standard $10 \mathrm{~ns}$, and the transmitter cabling was optimized for fastest derivative impulse radiation, but without overvoltage burnout of the $4015 \mathrm{~B}$ remote pulse head. This was done by ensuring that the time-domain reflections in the transmitter system were carefully delayed relative to the main generator pulse delivered to the antenna. The four impulse excitations were formed 
by inserting optional PSPL 5910 risetime filters. Without a risetime filter, the fastest system impulse waveform had a $50 \%$ pulse duration (full-width at half-maximum) of $48 \mathrm{ps}$, as captured with the Tektronix SD-32 50-GHz sampling head.

To estimate the pulse duration of the actual radiated electromagnetic wave, we can use simple Gaussian-system quadrature analysis to estimate the pulse duration of the radiated field incident on the wall. This is commonly applied to the transition duration (10-90\% risetime) of step waveforms but it is equally valid for the pulse duration ( $50 \%$ level) of impulse waveforms. The pulse duration of the electromagnetic wave is degraded or slowed by the band-limiting effects of the receiver system (including the receive antenna, cabling, and sampling head), and it may be unfolded from the acquired waveform. The acquired or measured pulse duration, $t_{w}\left(V_{r}\right)$, which is that of the received-voltage waveform, is modeled by the squared sum of the actual pulse duration of the impulse electromagnetic wave, $t_{w}(E)$, and the pulse duration of the receiving system, $t_{w}(r x): t_{w}^{2}\left(V_{r}\right)=t_{w}^{2}(E)+t_{w}^{2}(r x)$. Note that for a Gaussian component, the pulse duration of the impulse response is $92 \%$ of the transition duration of the step response (its' time integral). This permits us to use the step response of the receiving antenna and oscilloscope to estimate the impulse response of the receiving system. From the Appendix, the TEM horn antenna has a 20-ps transition duration for the step receiving response, and the SD-32 sampling head has an approximate transition duration of 9 ps. Thus, the combined receiving system step transition duration is about $22 \mathrm{ps}$, and the corresponding value of impulse pulse duration is $20 \mathrm{ps}$. Solving for the pulse duration of the radiated impulse electromagnetic wave,

$$
t_{w}(E)=\sqrt{\frac{1}{2}\left[t_{w}^{2}\left(V_{r}\right)-t_{w}^{2}(r x)\right]}=\sqrt{\frac{1}{2}\left[(48 \mathrm{ps})^{2}-(20 \mathrm{ps})^{2}\right]} \cong 44 \text { ps }
$$

This is the fastest impulse radiated wave which we have created thus far in our time-domain experimental program.

Without a risetime filter, the impulse system response had a pulse duration of $48 \mathrm{ps}$, as stated above. This is the pulse duration of the acquired received-voltage waveform, $t_{w}\left(V_{r}\right)$, and it includes the band-limiting effects of the receiver as well. With a 50-ps PSPL 5910 filter, the system pulse duration, $t_{w}\left(V_{r}\right)$, increased to $62 \mathrm{ps}$; with two 50-ps filters in series, the system pulse duration was $75 \mathrm{ps}$; and with a 100-ps filter, the resulting system pulse duration was $112 \mathrm{ps.} \mathrm{Thus}$ these four system configurations had a transient response range of 2.3 to 1 , with a corresponding variation in effective measurement system bandwidth. One of the experimental goals was to see what effect the pulse duration had on the transient propagation through the media of interest.

The experimental procedure was to: 1) measure the 'through' waveform for the four transmitter configurations; 2) move the transmitter system platform back into the high-bay room where the receiver platform was located; and then 3 ) carefully repeat the measurements for the free-space reference configuration. The instrumentation was powered down for the minimum time, and steps were taken to ensure the best measurement stability and repeatability for these sets of data acquisition. The critical settings for the CSA-803 sampling scope were: $500 \mathrm{ps} / \mathrm{div}$ (for a total time record of $5.12 \mathrm{~ns}$ ), 2048-point record lengths, 512-waveform averaging, and sampling head smoothing enabled. Both the pulse generator and scope settings were optimized for minimum time jitter and maximum data-acquisition rate, with a nominal repetition rate of 
$200 \mathrm{kHz}$. The transmit and receive antennas had to be carefully aligned, with the same aperture separation as for the 'through' setup. Because the time-domain antenna pattern variation is easy to see in the received voltage waveform, it was relatively simple to perform the boresight antenna alignment to better than $1^{\circ}$ accuracy.

Examining the equations for the low-loss analysis method shows two primary effects of antenna alignment errors. An error in antenna range/separation between the two setups results in an error in the time-domain insertion delay and a corresponding error in the phase shift of the computed transfer function, $H(f)$. This yields an error in the computed dielectric constant of the wall or layer under test. We estimate the worst-case range error to be $0.5 \%$, and since the dielectric constant is a squared function of the differential time delay, we estimate the dielectric constant error to be less than $1 \%$. The time-base accuracy of the pulse generator and sampling scope combination is much better than this level, so the antenna range error is the dominant contributor to estimation error in the dielectric constant. An angular error in antenna pointing results in an error in the relative spectral content between the two setups and a corresponding error in the magnitude of the transfer function, $|H(f)|$. This would then primarily affect the accuracy of the computed loss tangent versus frequency. Because the complexity of the time-domain antenna pattern makes this error analysis difficult, we aren't able to provide a simple characterization of this type of error. However, the data-acquisition errors of the sampling scope introduce the same effects, and they are at the $1 \%$ level; as a result we expect a worst-case bound of $5 \%$ or less for the estimation error in the transfer function magnitude or derived loss tangent.

It should be pointed out that for the low-loss analysis method (Equations 10-12), the error in computed dielectric constant propagates into the error for the loss tangent because the loss tangent is based on both $|H(f)|$ and $\varepsilon_{r}(f)$. This means that the error in loss tangent will be cumulative and greater than that in the dielectric constant or $|H(f)|$. The other primary error would be that due to the assumption of plane-wave propagation in the model, whereas spherical-wave propagation is more accurate. Bounding this model error was beyond the scope of this first experimental work.

Figure 8 shows one set of data for the Sandia wall, using the fastest impulse excitation with 48-ps system duration. The free-space reference waveform and the 'through' waveform are shown after pre-processing and normalization to the peak value of the reference waveform, for easy comparison. The raw acquired waveforms had the leading-portion of the pulse baseline zeroed to remove scope offset drifting, and the late-time portion was carefully tapered to zero level using a custom extended-cosine-bell time-domain window in order to provide the cleanest measurement of the single-pass transient response of the wall. Some of the complicating factors which determine the duration of the measurement clear time for this single-pass measurement are: an antenna round-trip clear time of $6 \mathrm{~ns}$ (due to their length); radiated pulse bounces between the wall and each antenna; and ringing inside the wall. One distinct advantage of time-domain metrology is the fact that it offers a straightforward way of capturing the transient causal response, at least for some time-duration limited period. A good understanding of the hardware timing and propagation (physics) timing is necessary in order to obtain the best time-domain experimental data. 


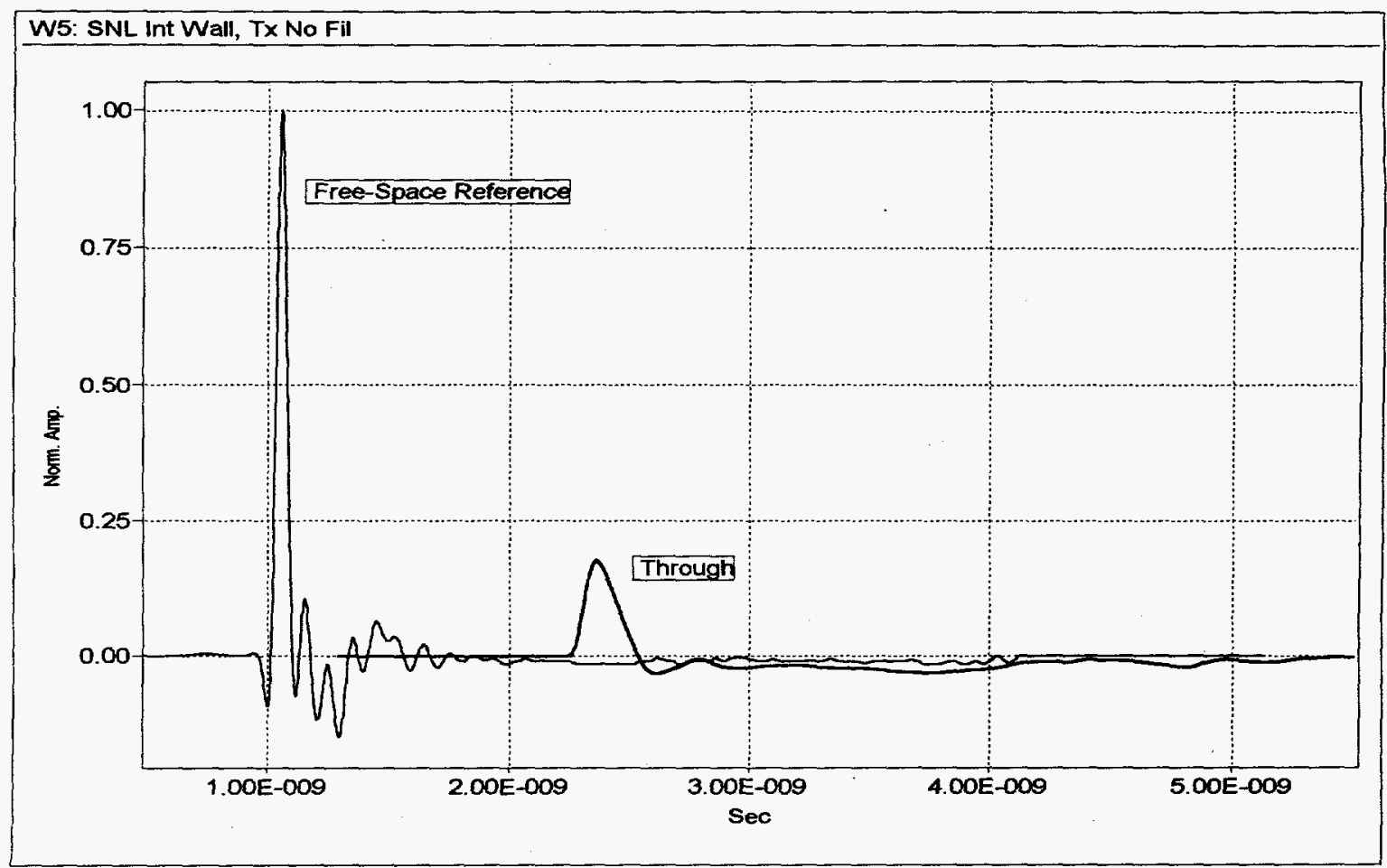

Figure 8. Free-Space Reference and 'Through' Waveforms for 48-ps Pulse-Duration Measurement of Sandia Concrete Wall; Timescale is $1 \mathrm{~ns} / \mathrm{div}$.

Note that even though the peak-normalized pulses in Figure 8 are positive-going, the raw waveforms were negative-going due to the negative step transition of the 4015B pulse generator. Note too the 1.3-ns differential time delay due to pulse retardation in the wall. This was discussed in some detail for the Series-1 data. Note as well that the 'through' pulse shows a strong lowpass-filtering effect, which will be explicitly seen in the transfer function later on.

The data for the other three measurement configurations are shown in the next figures - the 62-ps system pulse duration data is shown in Figure 9, the 75-ps data is given in Figure 10, and the 112-ps data is shown in Figure 11. Comparing the free-space waveforms in these four figures, note the increasing value of $t_{w}\left(V_{r}\right)$, the $50 \%$ pulse duration. Comparing the 'through' waveforms, it is interesting to see that the relative pulse attenuation decreases as $t_{w}\left(V_{r}\right)$ slows down (increases). This is because the faster pulses have higher-frequency spectral content which is attenuated more severely than the lower-frequency content of the slower pulses. The additional time delay for the 'through' impulses is very consistent also, about $1.3 \mathrm{~ns}$ for the $30.5-\mathrm{cm}$ thick wall.

As explained in the section on our radiated transmission measurement technique, this electromagnetic propagation phenomenology can be couched in terms of a linear two-port network or system. And a complete description is contained in the complex frequency-domain transfer function, $H(f)$, which requires a deconvolution operation in its' determination or computation. Alternatively, a two-port network can be represented by a time-domain quantity, 


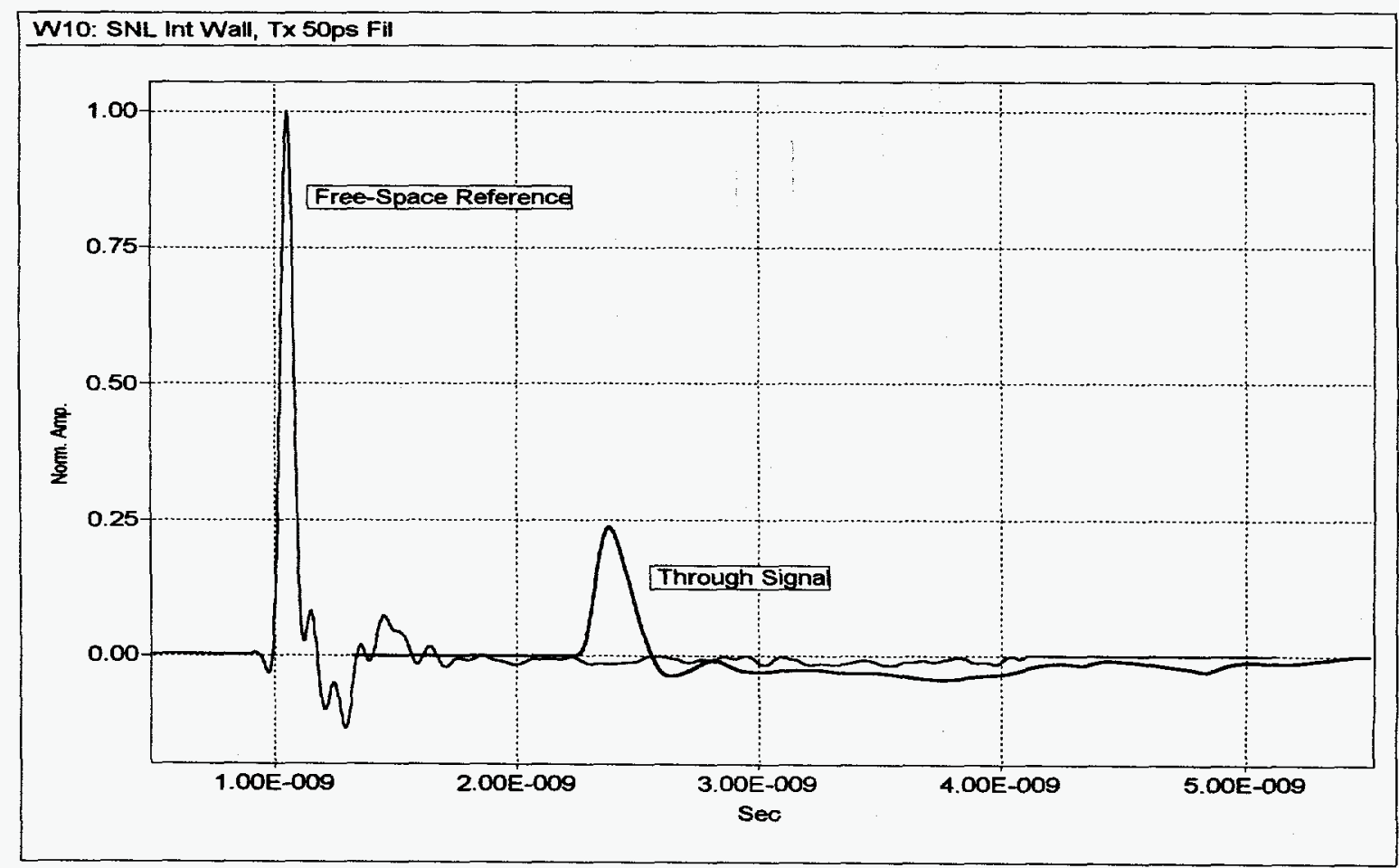

Figure 9. 62-ps Free-Space and 'Through' Waveforms for Sandia Concrete Wall; 1 ns/div.

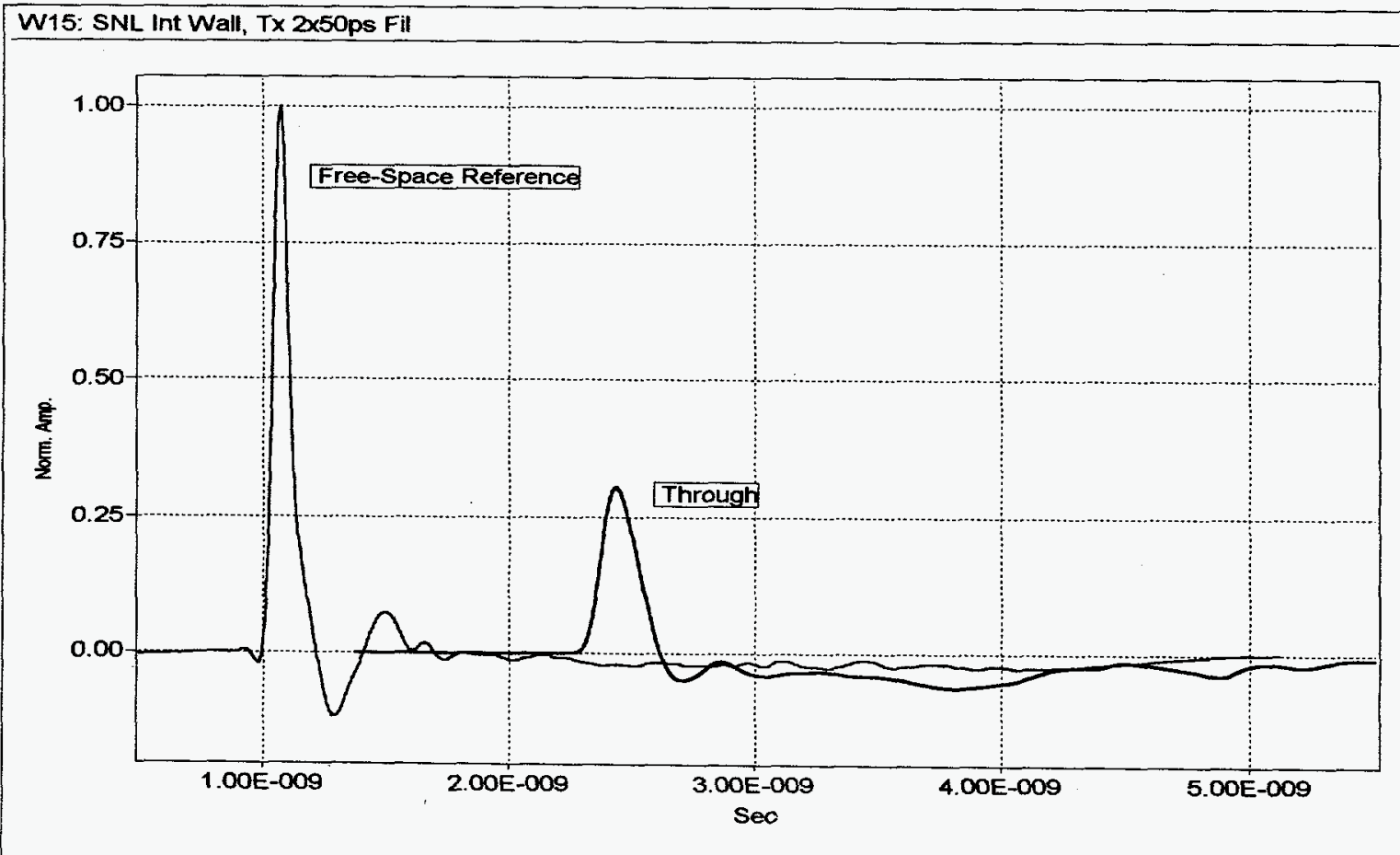

Figure 10. 75-ps Free-Space and 'Through' Waveforms for Sandia Concrete Wall; $1 \mathrm{~ns} /$ div. 


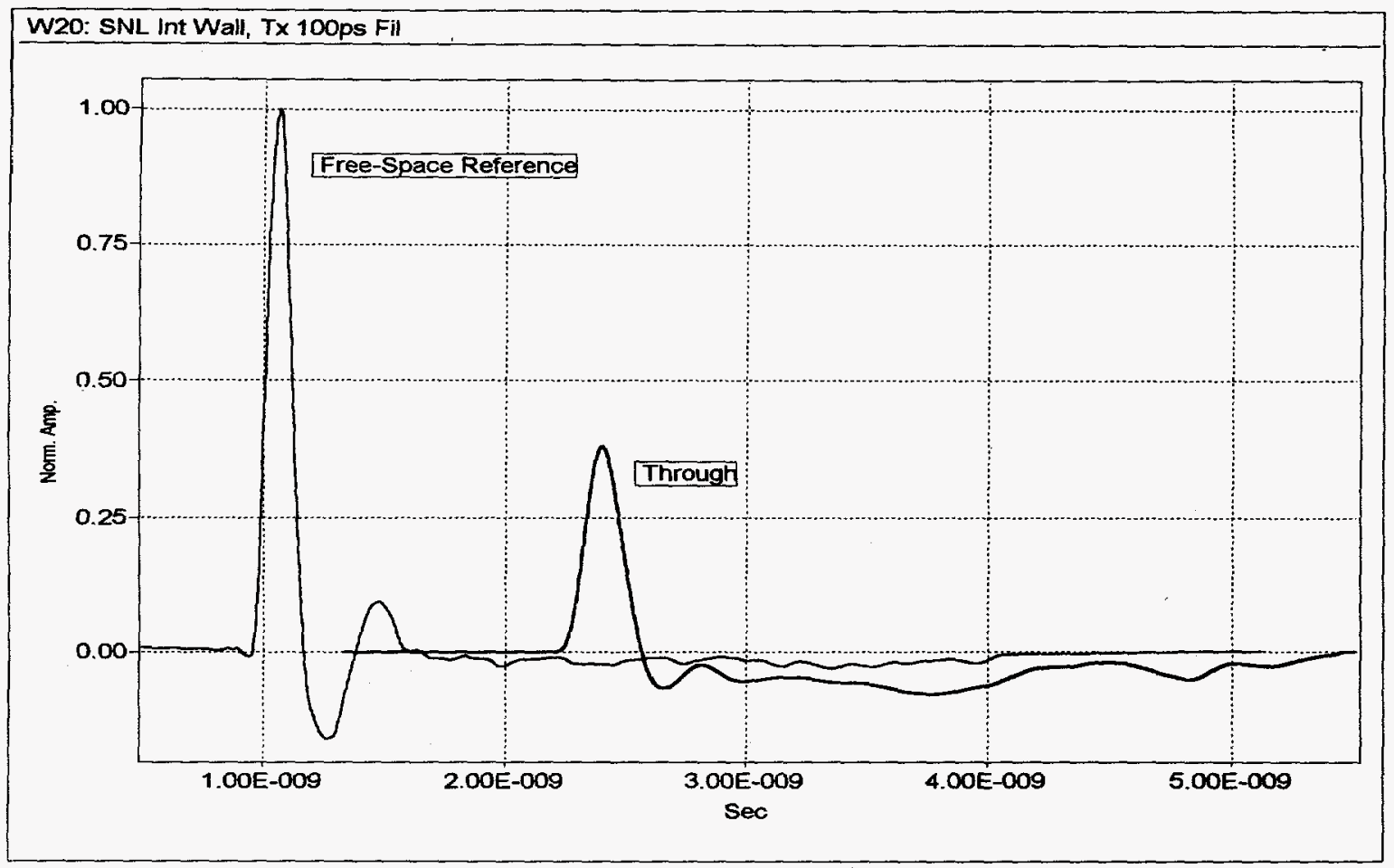

Figure 11. 112-ps Free-Space and 'Through' Waveforms for Sandia Concrete Wall; $1 \mathrm{~ns} /$ div.

the cross-correlation function. If $x(t)$ represents $E_{t 2}^{f s}(t)$, the received wave for the free-space reference configuration, and $y(t)$ represents $E_{t 2}(t)$, the received wave through the layer or wall, then the cross-correlation function is defined to be

$$
R_{y x}(\tau) \equiv \frac{1}{2 T} \int_{0}^{2 T} x(t+\tau) y(t) d t,
$$

where $T=N \Delta t$ is the record length of the acquired signals.

This is a correlation integral versus time delay or lag between the excitation (our free-space reference pulse) and the response (our 'through' pulse). Bendat and Piersol (1993) and Brigham (1988) provide in-depth discussions of cross-correlation, which naturally yields a maximum response at the time delays of the network or system under test. This is especially useful for multiple-path propagation identification in the presence of noise, or for our problem of estimating the differential time delay of single-pass transient propagation through a layer. Additionally, Brigham (1988) is an excellent text on Fourier transforms and signal processing. Applying this cross-correlation to the four sets of data above, Figure 12 shows the cross-correlation of the free-space and 'through' pulses for the Sandia concrete wall. Even though there is some variability in the value of the maximum correlation, the corresponding value of differential time delay, $\Delta \tau$, is fairly consistent across the set of four impulse pulse durations. 


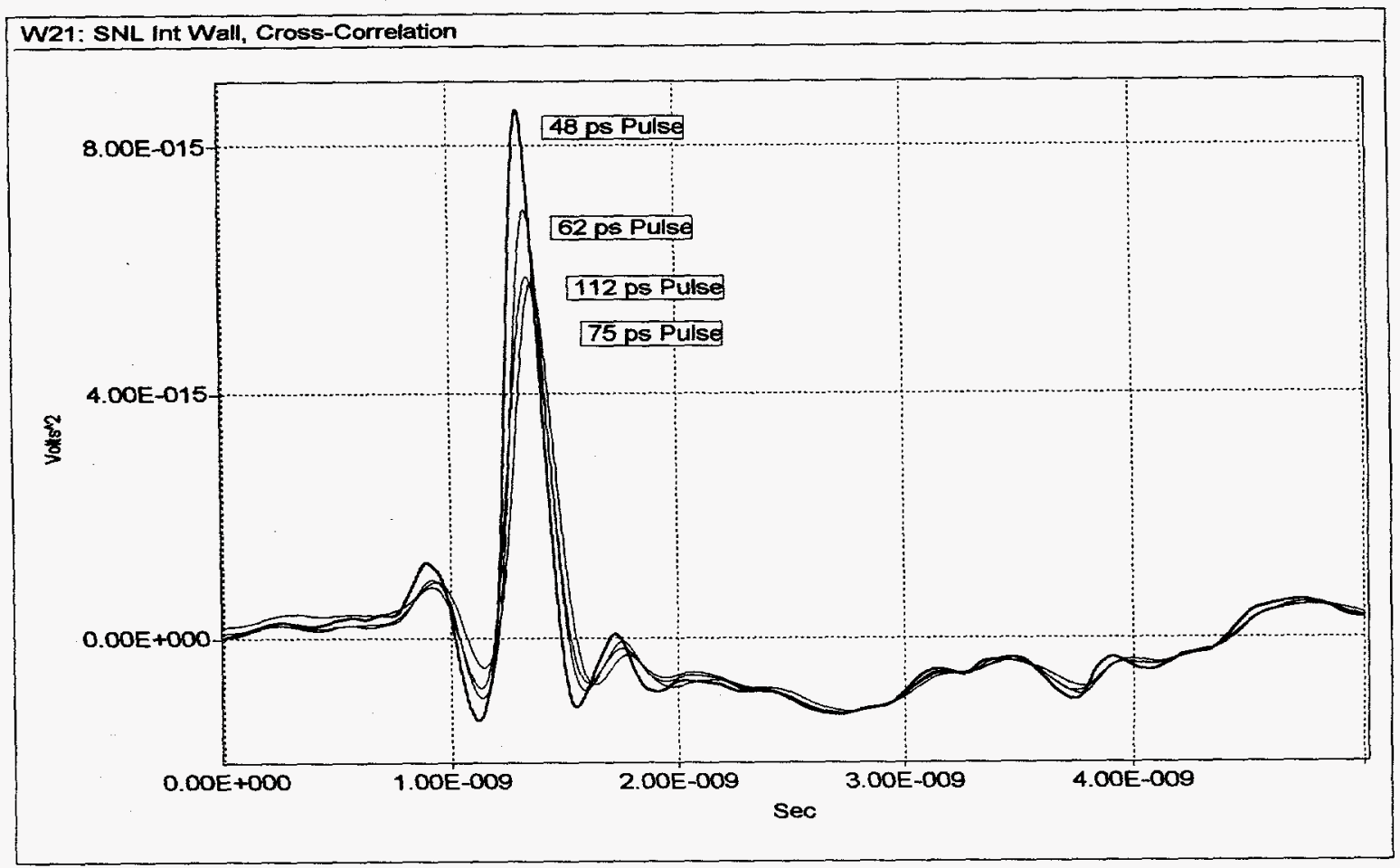

Figure 12. Cross-Correlation Function for Four Datasets of Sandia Concrete Wall; 1 ns/div.

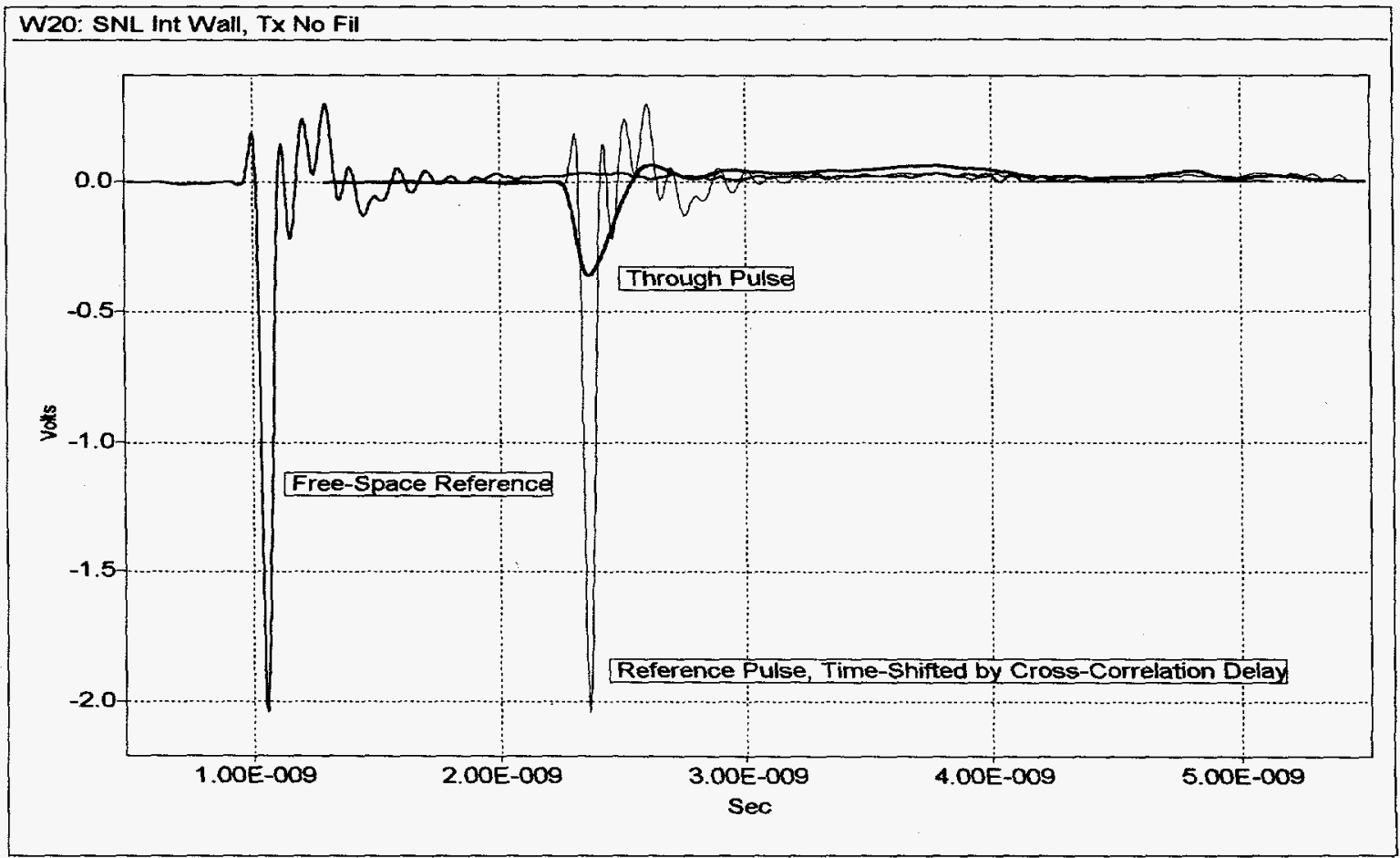

Figure 13. 48-ps Free-Space, Time-Shifted Free-Space, and 'Through' Waveforms for Sandia Concrete Wall; 1 ns/div. 
Figure 13 shows the fastest dataset for the Sandia wall (as in Figure 8), with an additional waveform - the free-space reference pulse which has been time-shifted by the maximum cross-correlation time lag $(1.30 \mathrm{~ns})$. These voltage waveforms are not peak-normalized, thus showing their negative-going nature. More importantly they show the obvious lowpass filtering effect of the wall.

Figure 14 shows the log-magnitude frequency-domain spectra of the free-space and 'through' waveforms for the 48-ps pulse-duration measurement. There is also an associated phase component versus frequency. Note that the spectral content of the system measurement is valid up to $>20 \mathrm{GHz}$, and that the spectral content of the 'through' pulse is much reduced in comparison (good up to about $12 \mathrm{GHz}$, where the spectrum goes into the noise floor). The resulting dynamic range for these time-domain measurements is about $40-50 \mathrm{~dB}$, a function of the 8-bit analog-to-digital converter of the sampling scope and the amount of waveform averaging employed in the acquisitions. Compared to vector network analyzers with a typical dynamic range of 80-100 dB, this illustrates a fundamental limitation of time-domain methods (smaller dynamic range).

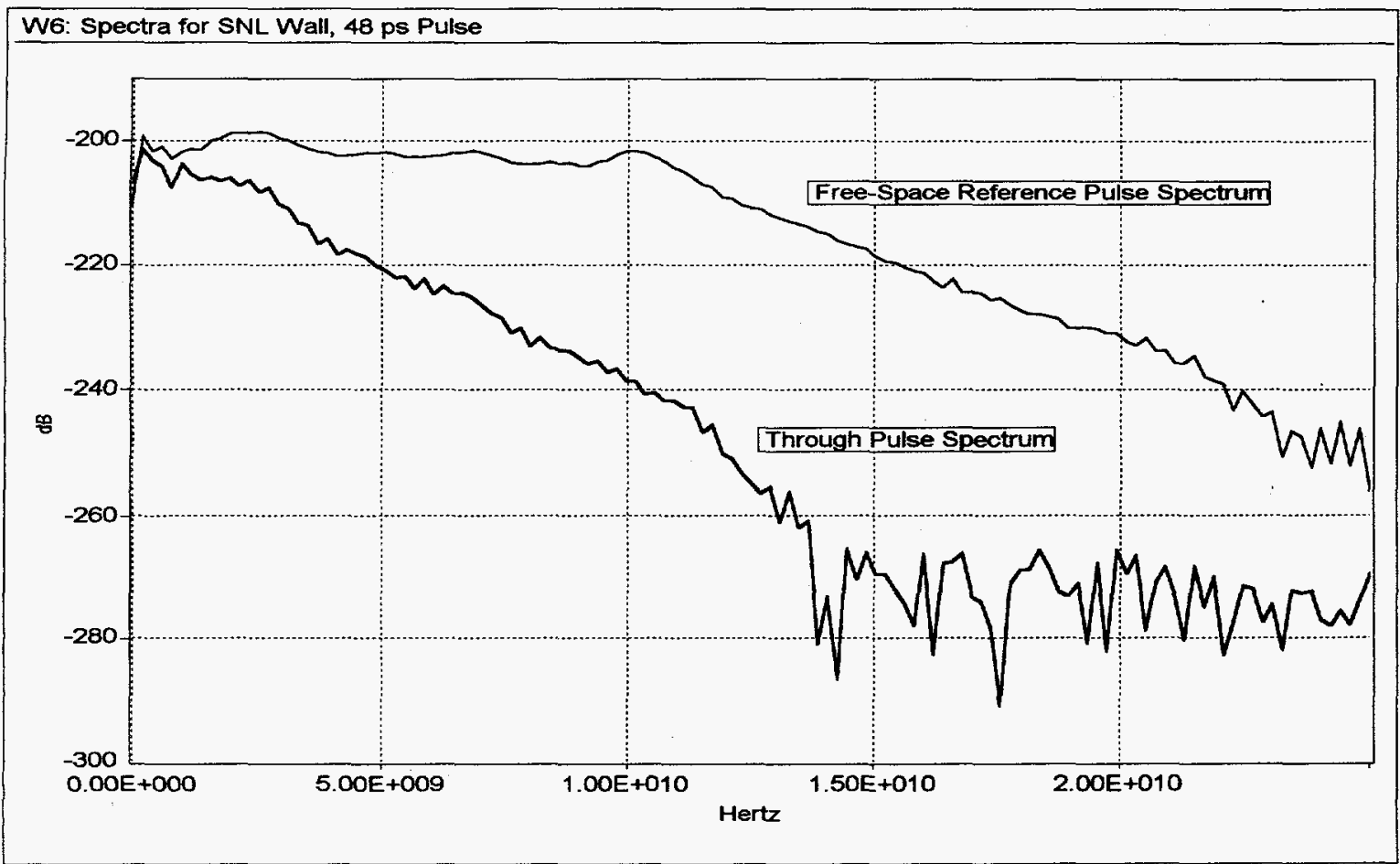

Figure 14. Frequency-Domain Spectra of Free-Space and 'Through' Waveforms for Sandia Concrete Wall, with 48-ps System Pulse Duration; Frequency Scale is $5 \mathrm{GHz} /$ div.

Based on the ratio of the 'through' pulse spectrum and the free-space pulse spectrum, the transfer function of the wall (Equation $9 \mathrm{~b}$ ) was computed for the four system pulse durations. The log-magnitude of $H(f), 20 \log |H(f)| \mathrm{dB}$, is shown in Figure 15. All the data depict a gradually increasing loss versus frequency, with the fastest impulse excitation (shown in bold) yielding the cleanest spectral characterization over the widest bandwidth (from UHF to $15 \mathrm{GHz}$ ). 
Corroborating swept-frequency measurements were made of the Sandia concrete wall with a Hewlett-Packard 8719A network analyzer. The analyzer was configured to provide the widest-bandwidth measurement of $S_{21}(f)$, the forward scattering parameter between the input port of the transmit antenna and the output port of the receive antenna. Stepped sweep mode was used, with 801 points from $150.0 \mathrm{MHz}$ to $13.5100 \mathrm{GHz}$, with a frequency spacing of $16.7 \mathrm{MHz}$. A relatively slow manual sweep time of $3 \mathrm{~s}$ was used for good phase-locking stability, and averaging of 16 sweeps was employed. The bandpass impulse time-domain mode was used to monitor the 'time-domain' view of the frequency-domain data. Unfortunately, because of time constraints during testing of the Sandia wall, we employed the crudest (but quickest) calibration method for the analyzer - simple display normalization. This required a preliminary $S_{21}(f)$ measurement of the coaxial cabling on the two analyzer ports, storage in internal memory, and then display enabling of later data divided by this system $S_{21}(f)$. Because this calibration method ignores several network analyzer error sources, the resulting measurement is relatively crude.

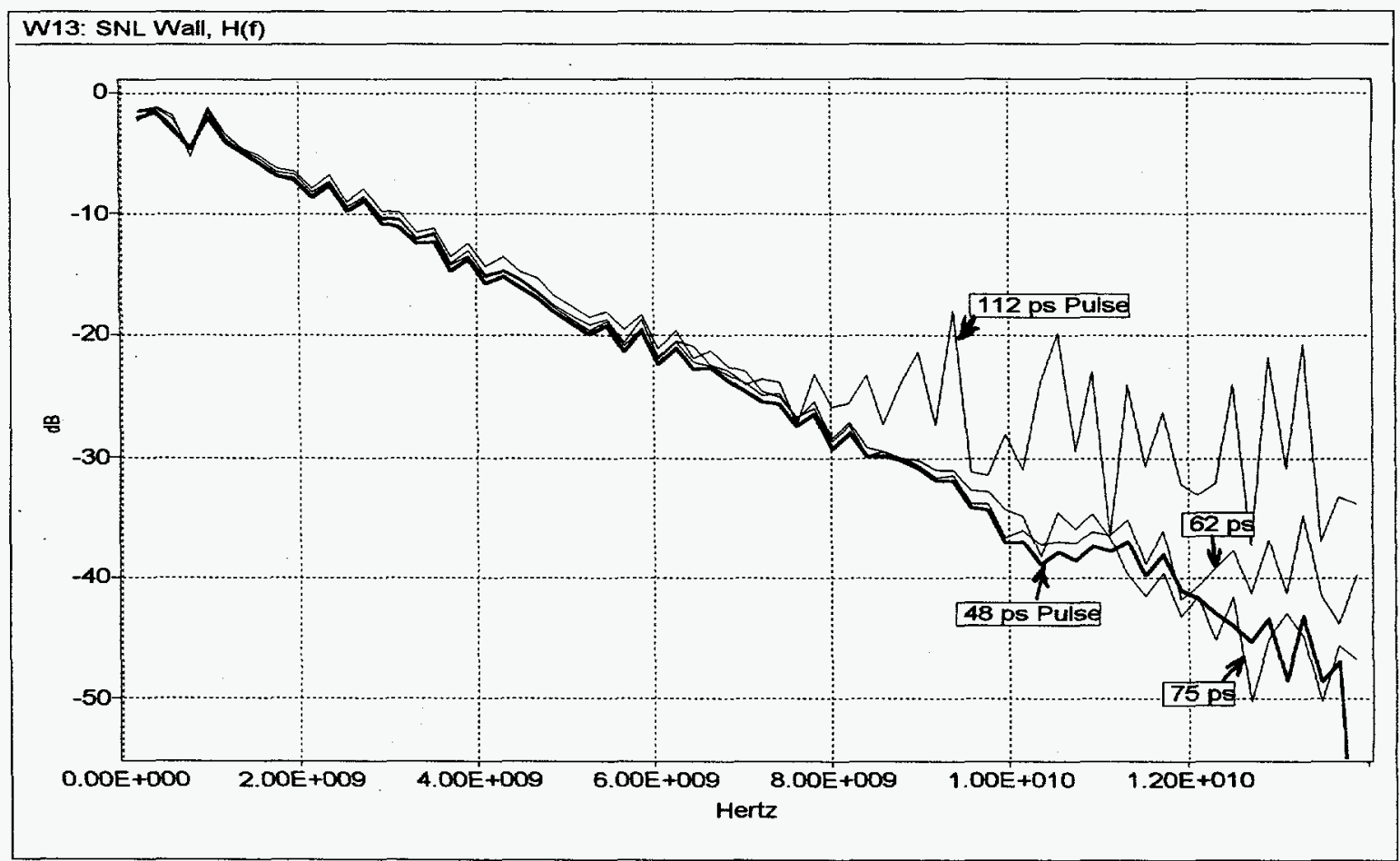

Figure 15. Log-Magnitude of Transfer Function of Sandia Concrete Wall, for System Pulse Durations of 48 ps, 62 ps, 75 ps, and 112 ps; $2 \mathrm{GHz} / \mathrm{div}$.

Figure 16 shows the log-magnitude of $S_{21}(f)$ for the free-space and 'through' configurations of the Sandia concrete wall. The free-space measurement actually characterizes the transmit and receive antenna combination, with increasing transfer of radiated energy versus frequency; this is consistent with the time-domain view of the transmit antenna having a derivative response and the receive antenna having a replica response. The 'through' plot of Figure 16 clearly shows the increasing wall attenuation versus frequency. 


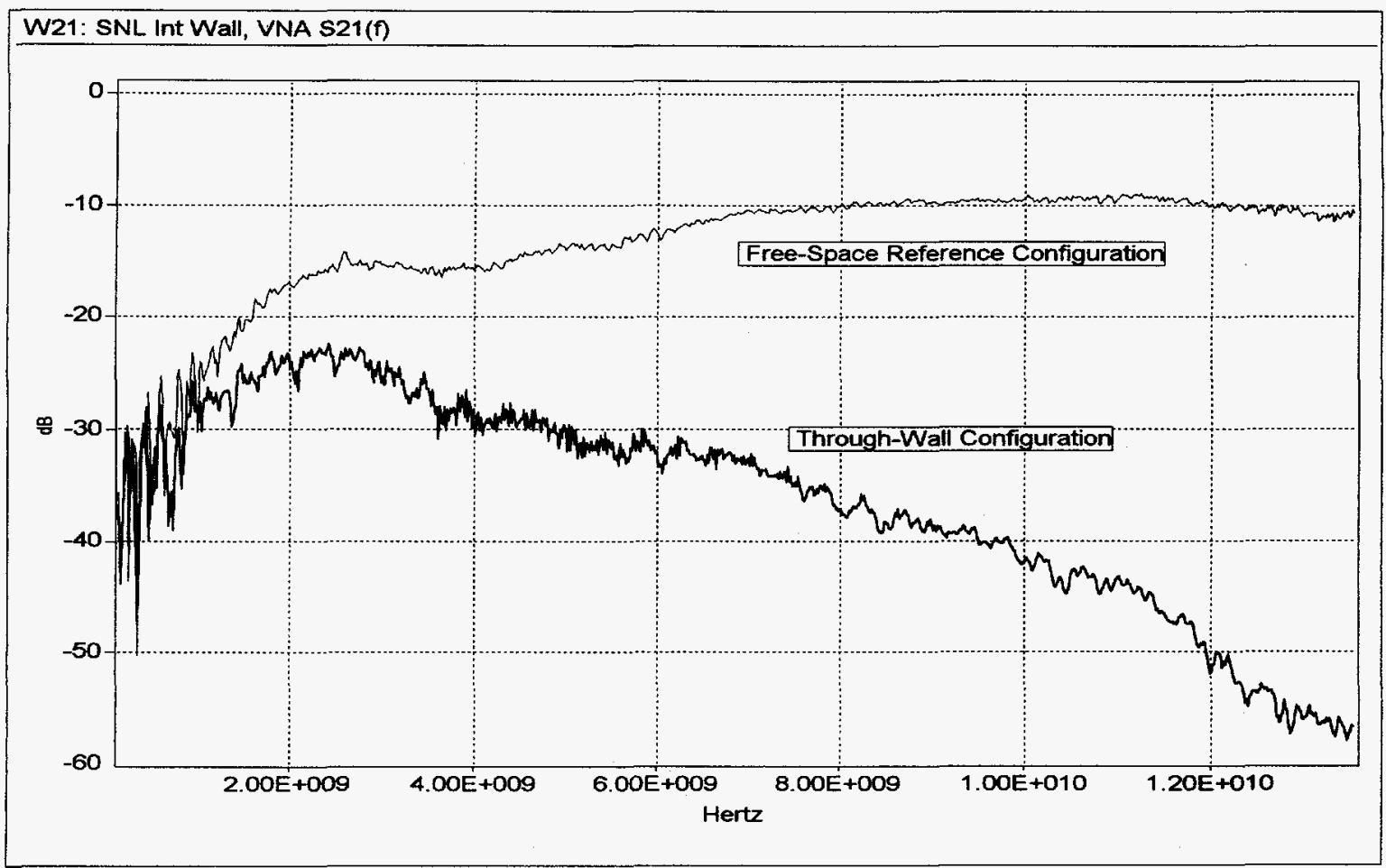

Figure 16. Log-Magnitude (dB) of Vector Analyzer Free-Space and 'Through' $S_{21}(f)$ Measurements for Sandia Concrete Wall; $150 \mathrm{MHz}$ to $13.51 \mathrm{GHz}, 2 \mathrm{GHz} / \mathrm{div}$.

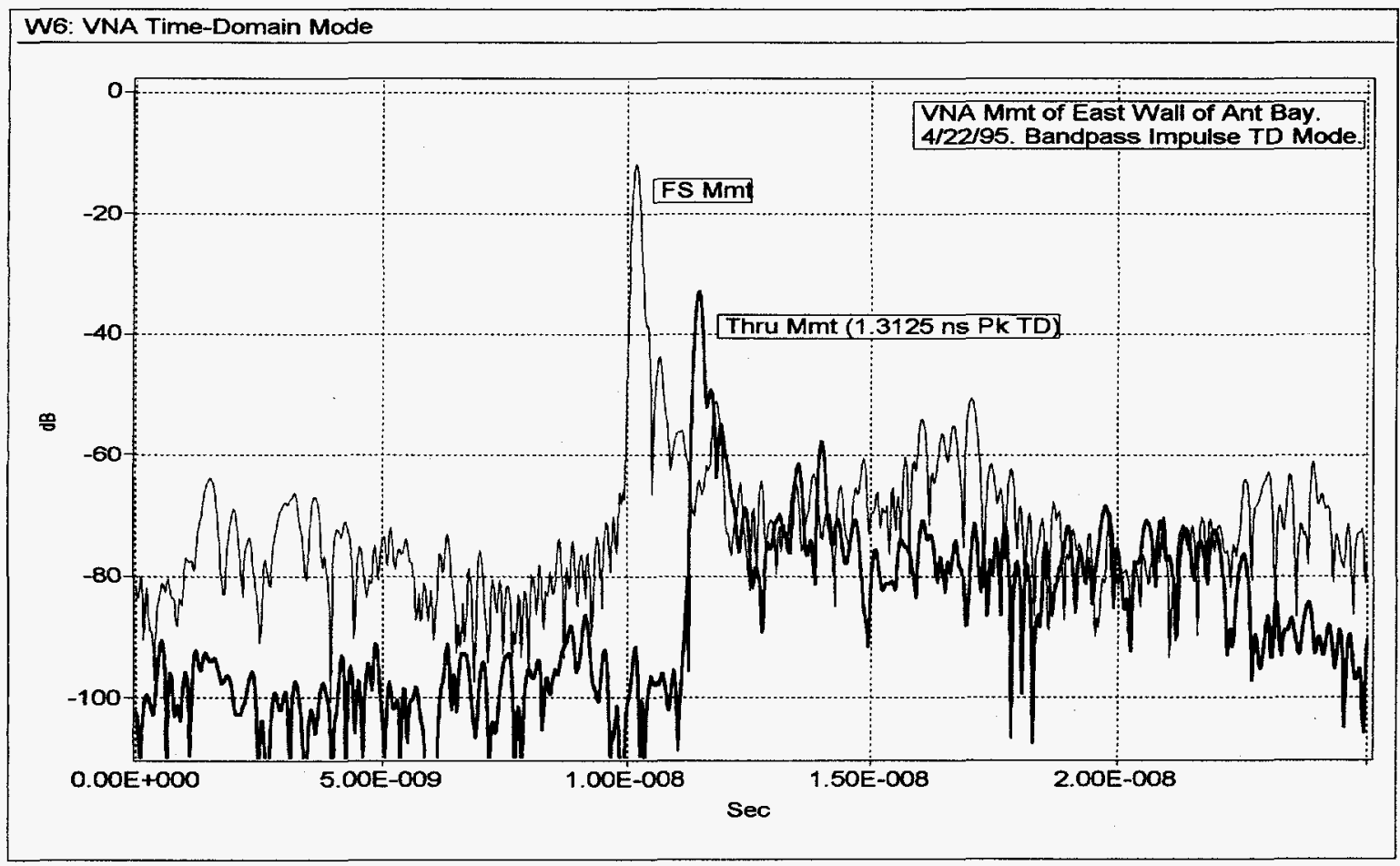

Figure 17. Bandpass Impulse 'Time-Domain' Data of Vector Analyzer Free-Space and 'Through' Measurements for Sandia Concrete Wall; 5 ns/div. 
Figure 17 shows the 'time-domain' data for the above network analyzer measurements, provided by an internal 'time-domain' mode in the $8719 \mathrm{~A}$ instrument. The relative amplitude of the swept-frequency measurement versus time is shown on a decibel scale. The two major peaks in this figure represent the single-pass received energy, with the first and largest peak corresponding to the free-space configuration. Notice that the time scale is absolute; the free-space peak occurs at just past $10 \mathrm{~ns}$ (' $1.0 \mathrm{E}-8 \mathrm{~s}$ '), which is the propagation time between the input port of the transmit antenna and the output port of the receive antenna. This includes the propagation time through each antenna and the propagation time for the radiated wave between the antenna apertures. The second peak, $1.3 \mathrm{~ns}$ later, corresponds to the single-pass wave for the 'through' configuration. This insertion delay agrees with the time-domain differential delay measurements. There are other peak features (in both plots) which occur later in time, but because they are at least $20 \mathrm{~dB}$ below the main single-pass features, their influence on the dominant frequency-domain information isn't that significant.

Figure 18 shows two magnitude plots of the transfer function, one based on the ratio of $S_{21}(f)$ for the free-space and the 'through' configurations of the $8719 \mathrm{~A}$ vector network analyzer measurements, and the other based on the 48-ps system duration time-domain measurements as shown in Figure 15. There is good agreement, especially considering that the swept-frequency data was acquired with a crude calibration routine and that no time-domain gating was employed in the network analyzer to 'clean up' the propagation data. This latter point is seen in the fine 'noisy' details of the swept-frequency transfer function. The large fine variations below $1 \mathrm{GHz}$ are due to the interference of the later-time features shown in Figure 17, primarily caused by internal ringing of the two antennas; this low-frequency antenna ringing is seen as well in the data of Figure 16.

Returning to the time-domain data and the transfer function of the wall as shown in Figure 16, we can then form the corresponding impulse response of the wall by taking the inverse Fourier transform of the transfer function. This is the pulse which would emerge from the concrete wall if excited with a perfect delta-function impulse TEM wave; in other words, the non-ideal time-domain characteristics of the measurement system have been removed from the waveforms.

Figure 19 shows the impulse response of the Sandia wall, based on the transfer function using the four system pulse durations. There are several items to note about these four waveforms. One is that the data are very consistent, an important attribute of good experimental techniques. Another is that the primary impulse response is replica in nature, and that the average $50 \%$ pulse duration was $146 \mathrm{ps}$. Thus, if a transient wave with faster components than this is passed through this wall, the fastest components of the emerging wave will be degraded to 146 ps or slower. This band-limiting effect has definite implications in designing time-domain systems which involve propagation through concrete walls. The last item to notice is the negative undershoot following the impulse portion of the response; we think that this is partially caused or influenced by scattering off the reinforcing bar inside the walls. Some experiments were performed with the Wright wall which strongly point to this hypothesis. In addition, this hypothesis is reasonable to expect: negative scattered fields due to conducting scatterers, and time-delayed arrival due to the off-center position of the rebar relative to the propagation centerline. 


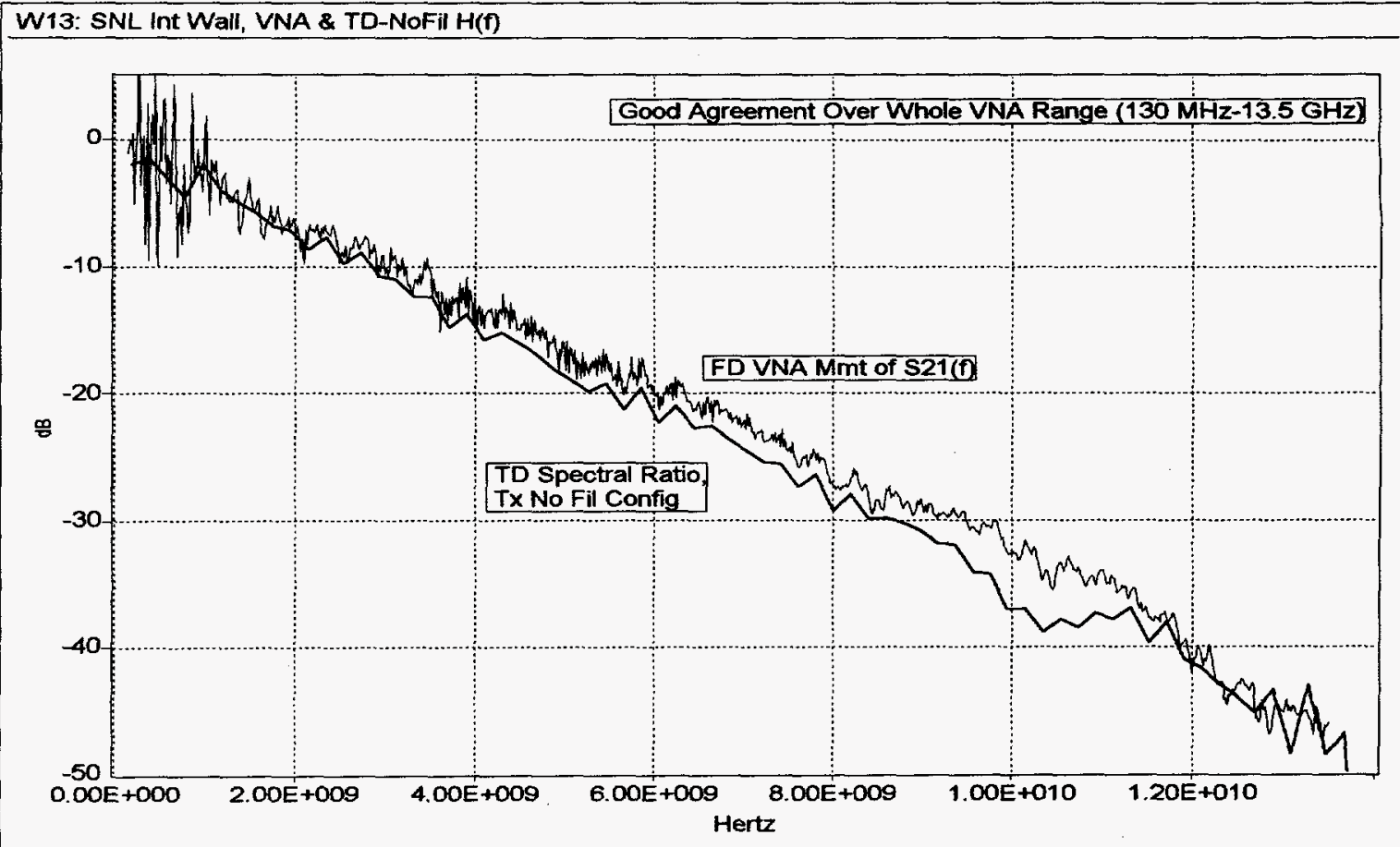

Figure 18. Comparison of Transfer Function Magnitude of Sandia Concrete Wall, for Time-Domain System (48-ps Pulse Duration) and Vector Analyzer System; 2 GHz/div.

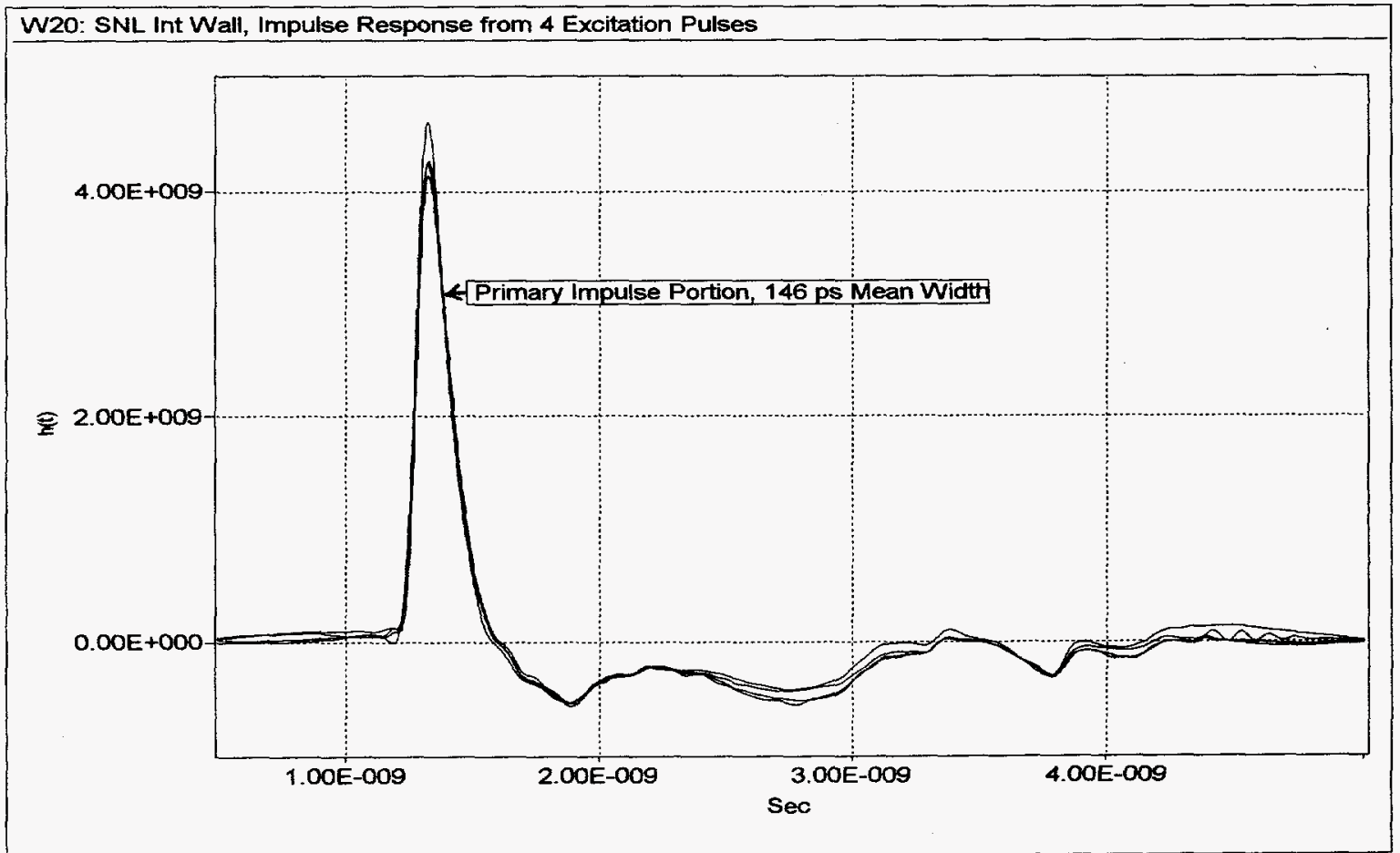

Figure 19. Impulse Response of Sandia Concrete Wall, for System Pulse Durations of $48 \mathrm{ps}, 62 \mathrm{ps}, 75 \mathrm{ps}$, and $112 \mathrm{ps} ; 1 \mathrm{~ns} / \mathrm{div}$. 
Using the low-loss analysis method embodied by Equations 10-12, we then derived the dielectric constant and loss tangent versus frequency from the transfer function. As a representative example, Figure 20 shows $\varepsilon_{r}(f)$ for the 48-ps pulse duration transient data, with clean/valid data for the $2-12 \mathrm{GHz}$ range. Overlaid is the simple time-domain parameter, based on the time lag to the maximum cross-correlation (Equation 13):

$$
\varepsilon_{r} \cong\left[1+\frac{\Delta \tau}{d / c}\right]^{2}=\left[1+\frac{1.3 \mathrm{~ns}}{30.5 \mathrm{~cm} / 30 \mathrm{~cm} / \mathrm{ns}}\right]^{2}=5.2,
$$

with fairly good agreement.

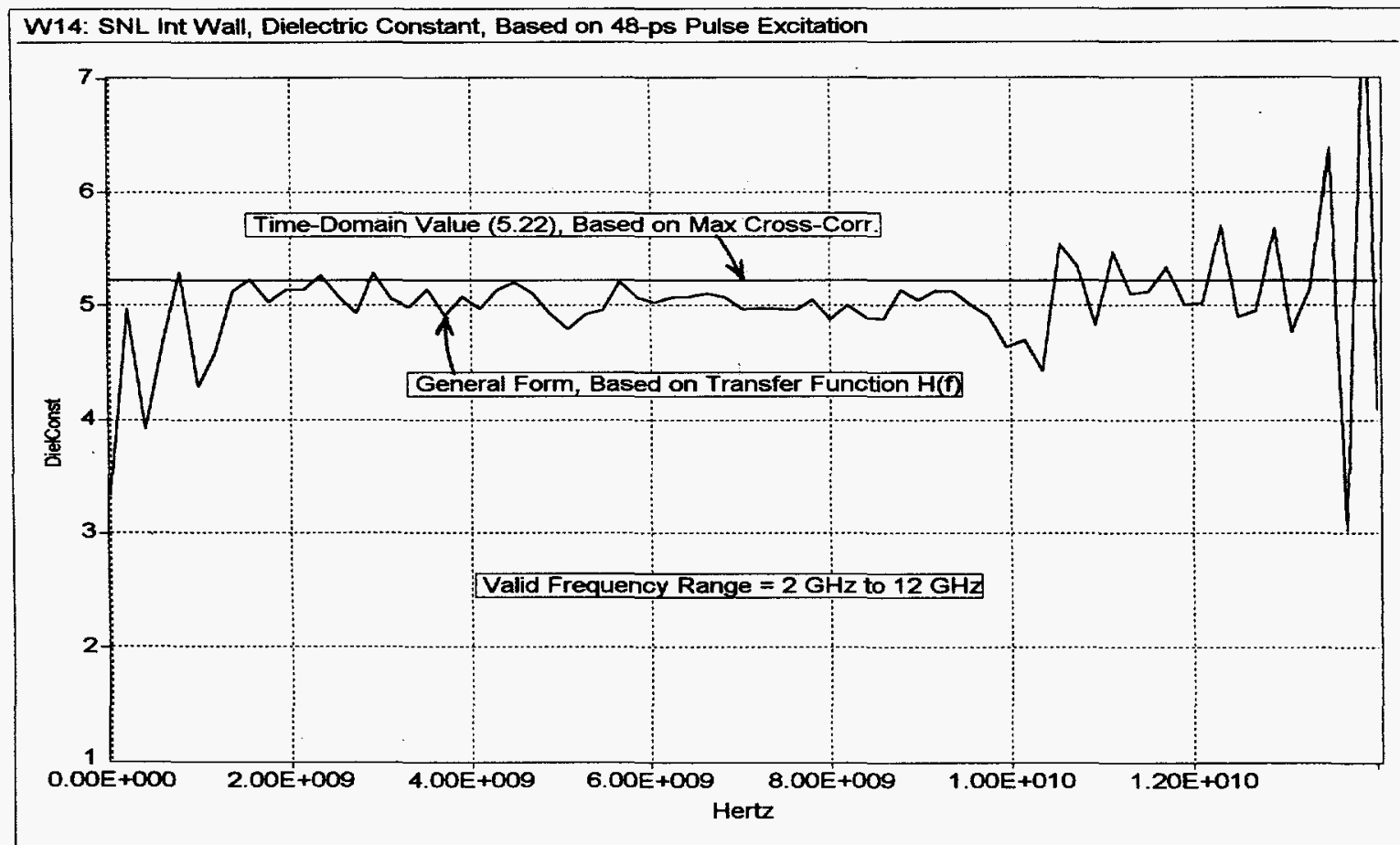

Figure 20. Dielectric Constant of Sandia Concrete Wall, Derived by Low-Loss Analysis Method from the Transfer Function of 48-ps Transient Data, Overlaid with Time-Domain Value from Maximum Cross-Correlation Lag; $2 \mathrm{GHz} / \mathrm{div}$.

The loss tangent, $p_{e}(f)$, was also derived from the transfer function (Equation 12), and Figure 21 shows this for the same 48-ps time-domain dataset. Note that it is fairly flat over the 2-12 GHz range, with an average value of 0.053 (a fairly low to moderate loss). It should be noted that the dielectric constant and loss tangent were consistent for the other three datasets as well.

Based on the dielectric constant and loss tangent computed above, the attenuation constant can then be evaluated with Equation 3:

$$
\alpha(f)=8.686 \frac{2 \pi f}{c}\left\{\frac{\varepsilon_{r}(f)}{2}\left[\sqrt{1+p_{e}(f)^{2}}-1\right]\right\}^{1 / 2} \mathrm{~dB} / \mathrm{m} .
$$




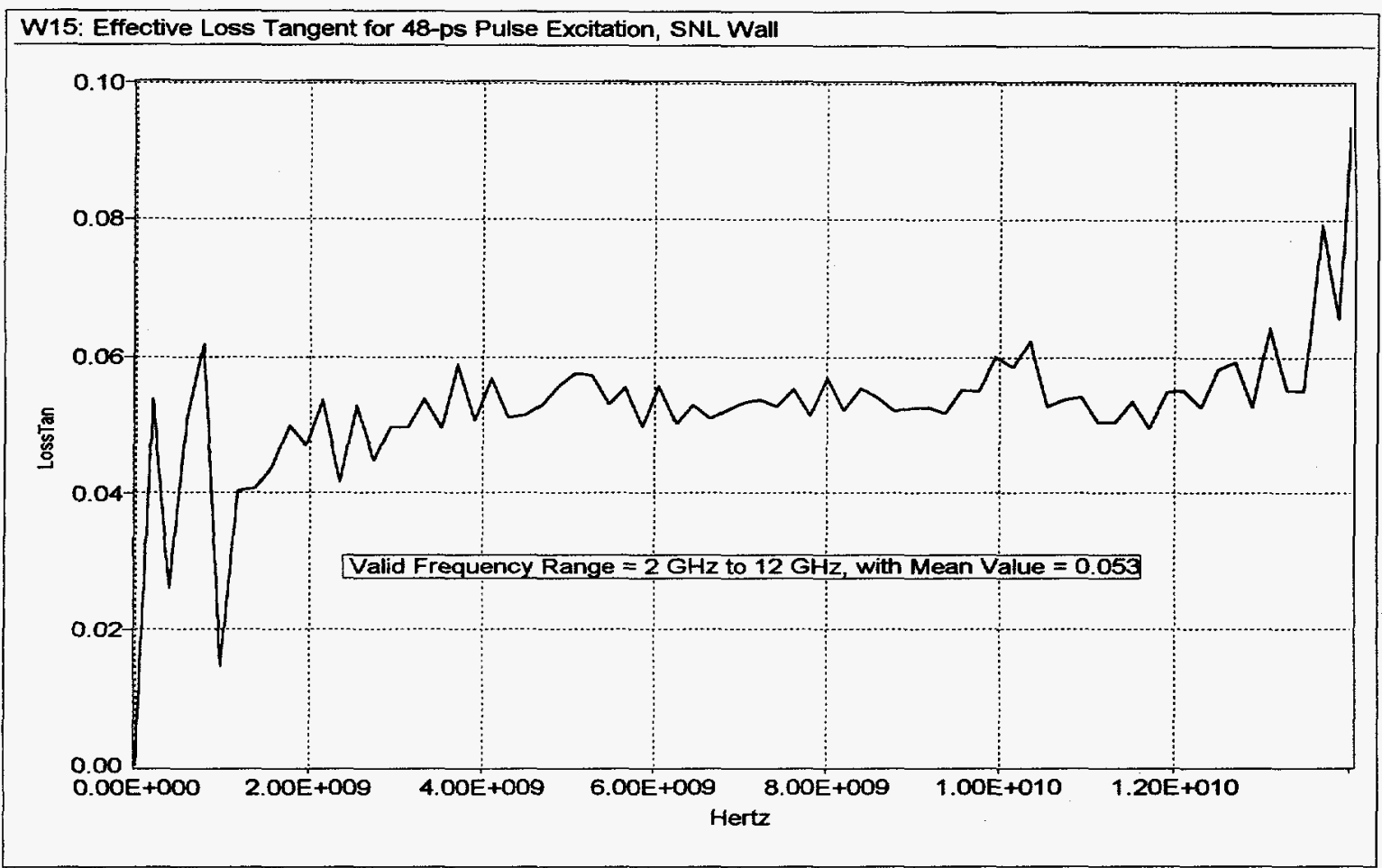

Figure 21. Loss Tangent of Sandia Concrete Wall, Derived by Low-Loss Analysis Method from the Transfer Function of 48-ps Transient Data; 2 GHz/div.

This indicates the attenuation of the field strength as a wave passes through the assumed homogeneous medium. Figure 22 shows the attenuation constant for the 48 -ps data, as well as the attenuation constant based on the Series-1 measurements. There were a variety of hardware (and software) improvements between the Series-1 and Series-2 experiments, with much better data obtained in Series 2. The attenuation constant for the Sandia concrete wall increases roughly linearly with frequency, with about $20 \mathrm{~dB} / \mathrm{m}$ at $2 \mathrm{GHz}$ and rising to about $110 \mathrm{~dB} / \mathrm{m}$ at $10 \mathrm{GHz}$. The upper bound for practical radar systems will be between these two points. 


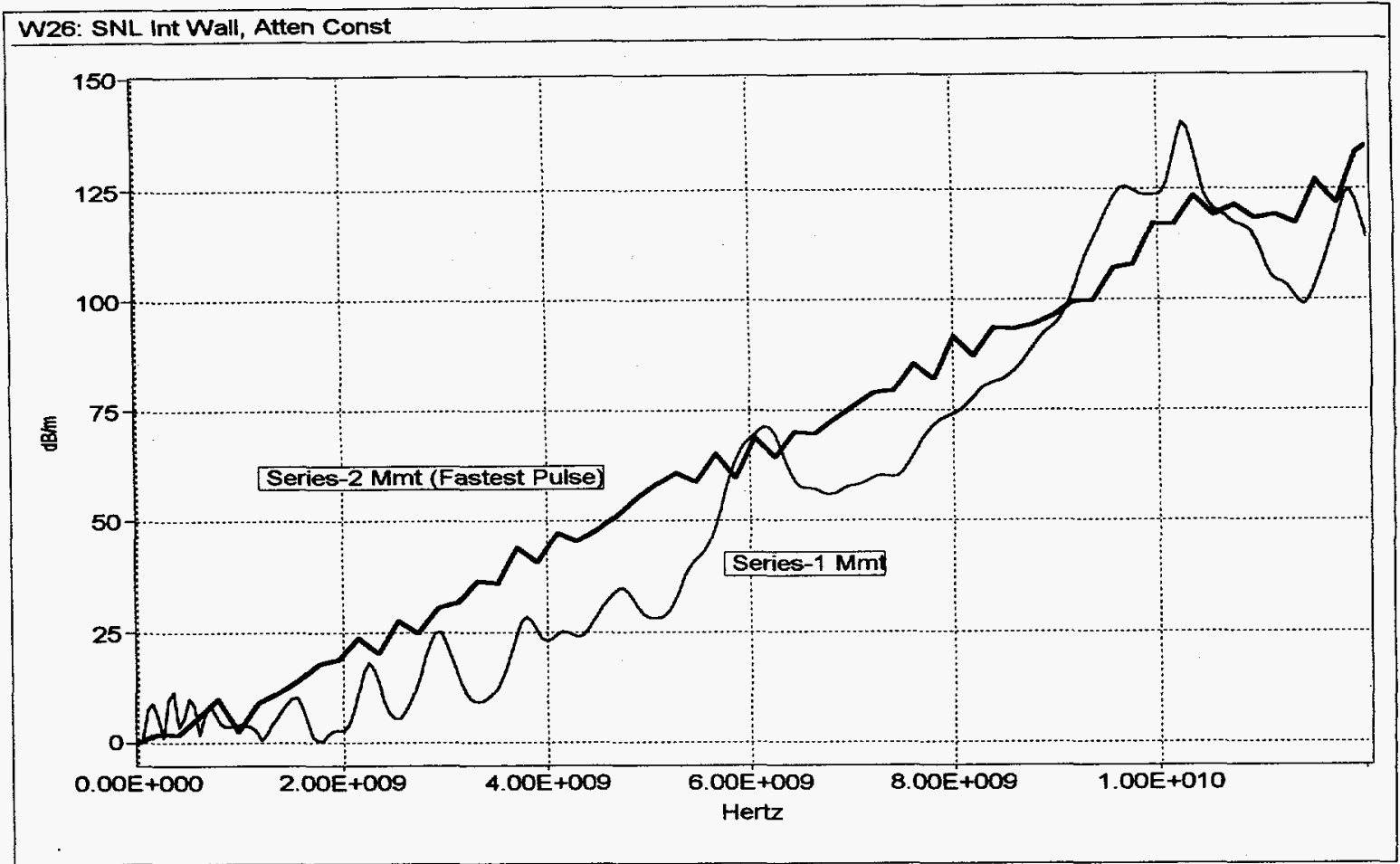

Figure 22. Attenuation Constant of Sandia Concrete Wall, Based on Series-2 Transient Data (48-ps System Pulse Duration) and on Series-1 Data; $2 \mathrm{GHz} / \mathrm{div}$.

\section{Wright Lab Concrete Wall}

The specific wall which we characterized at Wright Laboratory was the separating wall between Test Bays 16 and 17 in the Vitro Test Facility, attached to Bldg. 432 (on Eglin AFB). Figure 23 is a photograph of the experimental setup used to capture the free-space measurement system response. This was located in the hallway of the Vitro Test Facility, adjacent to the two test bays. The antenna aperture separation was $1.10 \mathrm{~m}$ (precisely the same as the separation for the wall measurement). The transmit antenna is pointed toward the viewer, with the pulse generator equipment located behind it. The receive antenna is pointed toward the transmit antenna, in this case with both main-beam boresight directions carefully aligned for maximum time-domain response. The receiving sampling oscillosope can be seen at the left side of the photo, sitting on top of a vector analyzer.

The Wright Lab experimental configuration consisted of the same receiver setup as used for the Sandia wall measurements (shown in the block diagram of Figure 7). But the transmitter system was modified for the Wright Lab work because the sandbox measurements required a longer clear time for the radiated field; we used an unmodified PSPL 4015B pulse generator which had a 10-ns pulse length rather than the 5-ns pulse length of the generator used for the Sandia wall work. Figure 24 shows a block diagram of the transmitter system used for the Wright Lab experiments. 


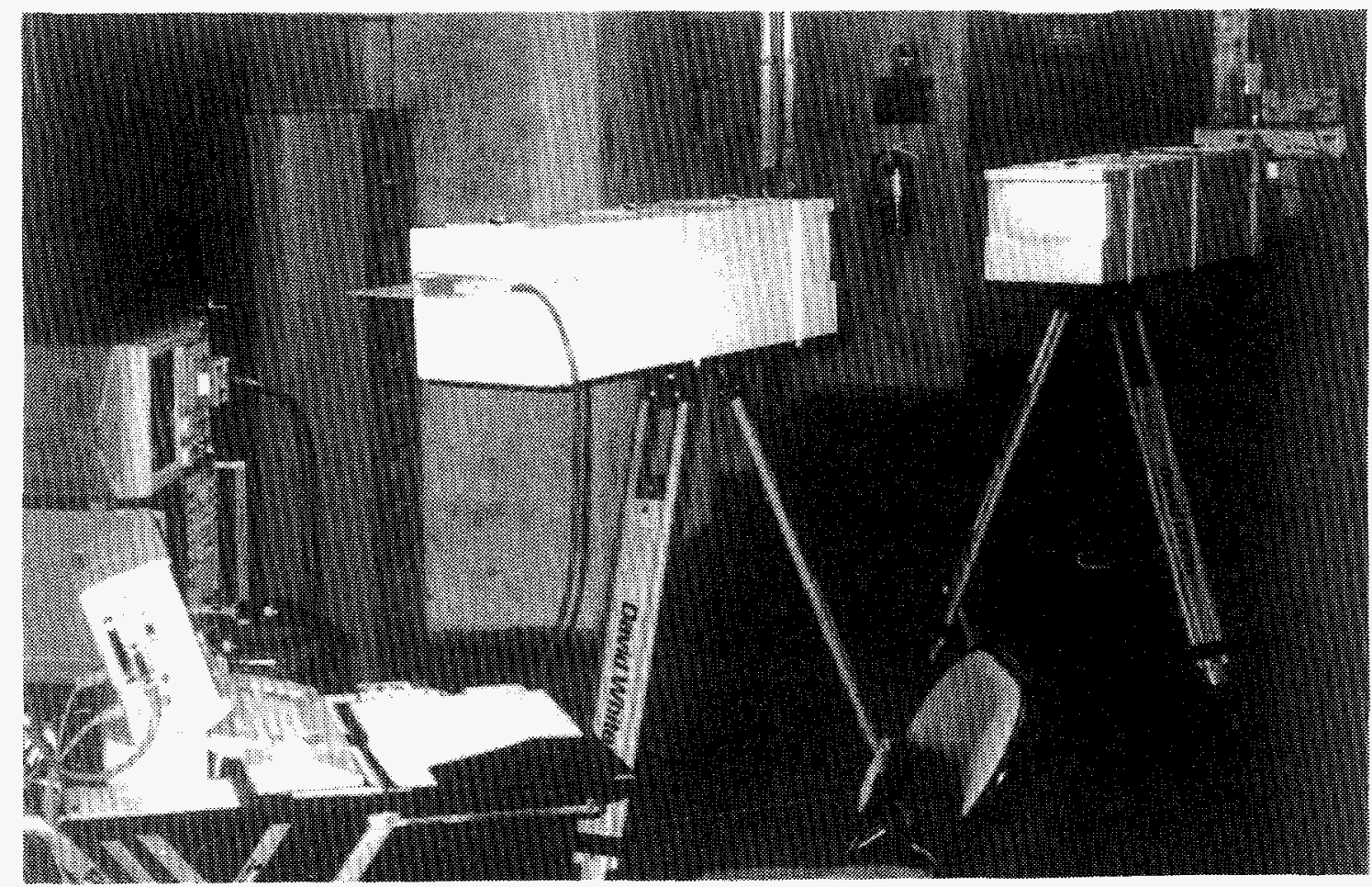

Figure 23. Wright Lab Experimental Setup of Free-Space Reference Measurement for Characterization of Concrete Wall.

Five different impulse waves and one bipolar wave were created by changing the transmitter components as follows. The fastest impulse was generated using the configuration of Figure 24, without a PSPL 5910 risetime filter. The system pulse duration for this setup was $48 \mathrm{ps}$, the same value as the impulse created for the Sandia wall experiments; however, the received system waveform was slightly different between the two transmitter configurations. A slightly slower system impulse was generated with an added 50-ps 5910 risetime filter, resulting in a 50\%-level pulse duration of $64 \mathrm{ps}$. A third system impulse, with a pulse duration of $84 \mathrm{ps,} \mathrm{was} \mathrm{made} \mathrm{by}$ removing the 4015B remote pulse head and using two 50-ps risetime filters. The fourth impulse had a pulse duration of $115 \mathrm{ps}$, using the $4015 \mathrm{~B}$ pulse head and a 100-ps risetime filter. The

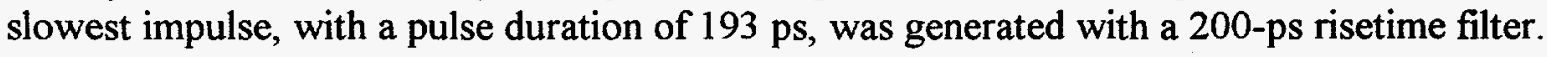

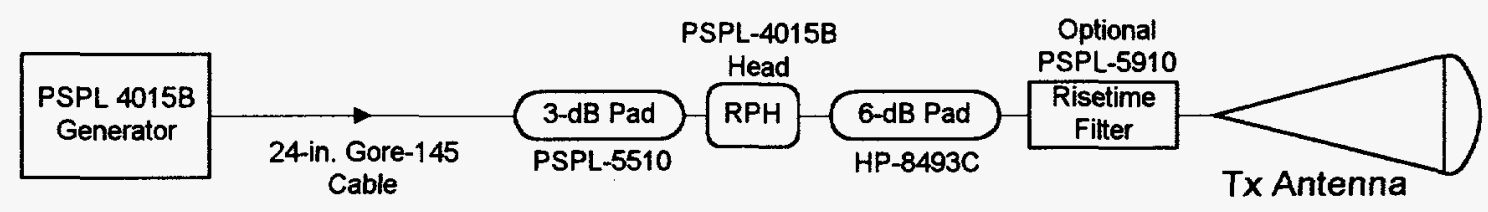

Figure 24. Block Diagram of Transmitter for Wright Lab Measurements.

Thus, five system impulses were generated and used to characterize the Wright wall, with pulse durations of $48,64,84,115$, and 193 ps. This offered a 4:1 range in transient excitation times, in order to see if this variation had any effect on the propagation performance of the concrete wall. In addition, a bipolar wave was created by using a PSPL 5208 impulse forming 
network between the 4015B remote pulse head and the transmit antenna (in place of the 6-dB pad and risetime filter).

For the 'through' measurements, Figures 25 and 26 show the transmitter and receiver setups respectively. In Figure 25, the vertically-polarized transmit antenna (in Test Bay 17) is oriented at normal incidence to the wall, with the aperture separated $40 \mathrm{~cm}$ from the front surface. In Figure 26, the receive antenna (in Test Bay 16) is seen in a similar orientation, on the opposite side of this wall. It is also easier to view the receive equipment in this figure; note that the sampling head is located close to the antenna port, in order to minimize cable degradation of the receive waveforms. The antennas were very carefully positioned in order to have a normally-incident propagation line-of-sight and repeatable wall separations (to an accuracy of better than $0.5 \mathrm{~cm}$ ).

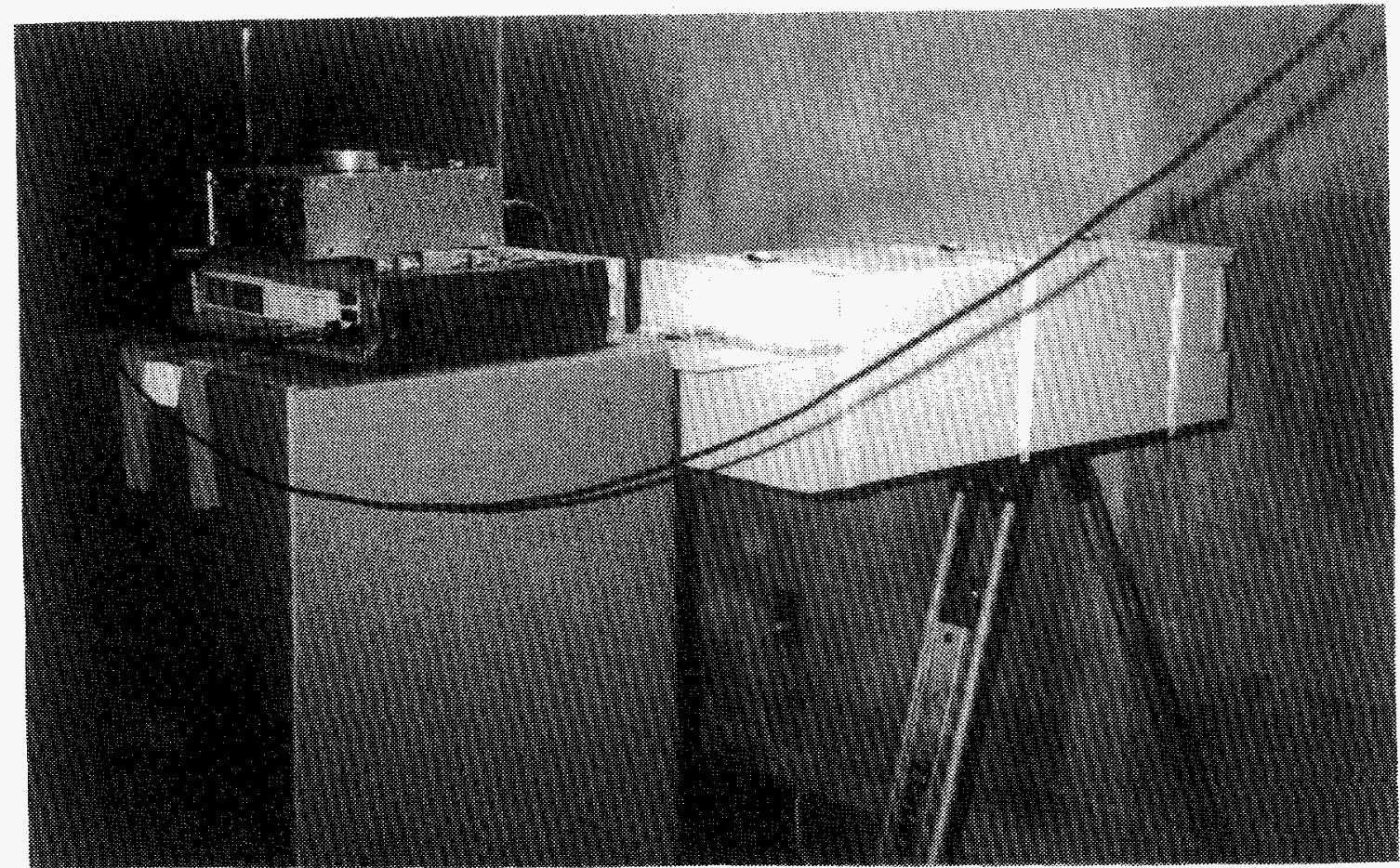

Figure 25. Transmitter Setup for 'Through' Measurement of Concrete Wall at Wright Lab.

Before the precise 'through' measurements were made, some time was spent studying the effects of the internal rebar in this wall. Blueprints of the building (dated 1973) were examined; there are two vertical rebar layers, with a horizontal spacing of 12 in., and their actual location horizontally in the wall was shown. There is one layer of horizontal rebar, with a nominal vertical spacing of 12 in. as well, but their position was not shown. Because the antennas were oriented for vertical linear polarization, we didn't expect the horizontal rebar scattering to be as significant as that of the vertical rebar; consequently, we concentrated on the vertical rebar effects. The propagation centerline was $1.22 \mathrm{~m}$ above the concrete floor (in both rooms). 


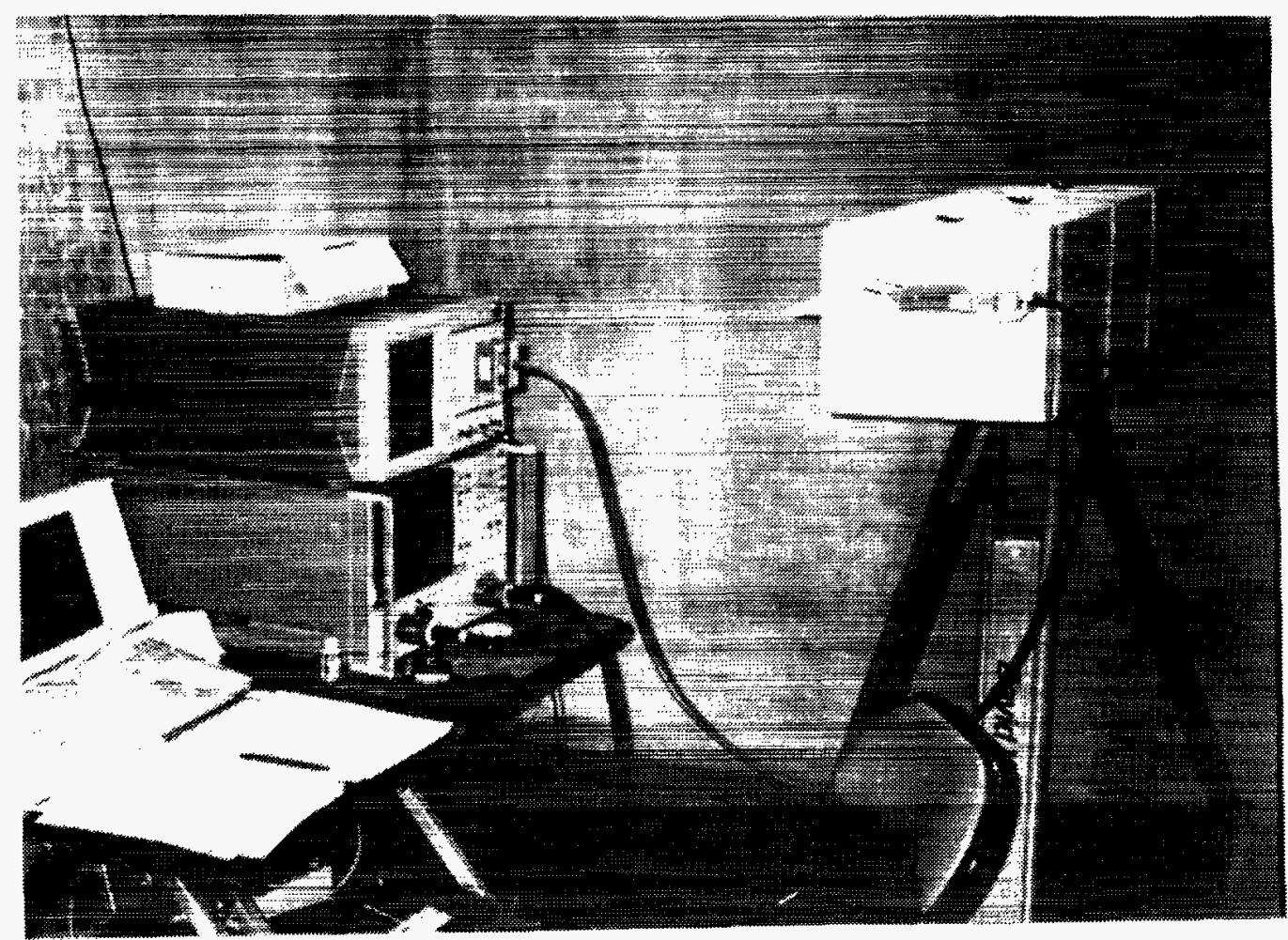

Figure 26. Receiver Setup for 'Through' Measurement of Concrete Wall at Wright Lab.

Two experiments were performed: we varied the horizontal propagation path centerline (by horizontally moving the antenna pair); and we taped a number of $1-\mathrm{m}$ long straight wires on the wall, located at the supposed positions of the internal vertical rebar. Figure 27 shows one of the scattering-wire configurations, with wires taped in position over the nearest four vertical rebar locations. Here the antennas were centered between the vertical rebar locations, so that the rebar and scattering wires would be symmetrically placed to the left and right of the propagation centerline. The closest pair of wires caused a slight attenuation in the direct impulse passing through the wall, and enhancement of the negative undershoot immediately following the primary impulse. The farther pair of wires caused a similar enhancement in the negative undershoot but later in time. With all four wires present (as in the photograph), both changes were observed, indicating simple superposition of the scattering.

Figure 28 shows the dataset for the fastest impulse system (48-ps pulse duration), with the 'through' waveform experiencing time delay, attenuation, and dispersion relative to the system reference. The free-space waveform for the Wright wall measurement is different than that of the Sandia wall measurement (shown in Figure 8) because a different transmitter system was employed. They have the same impulse, with a pulse duration of $48 \mathrm{ps}$, but the other waveform features differ.

The other time-domain datasets are shown in Figure 29 (64-ps impulse system), Figure 30 (84-ps impulse), Figure 31 (115-ps impulse), Figure 32 (193-ps impulse), and Figure 33 (bipolar 


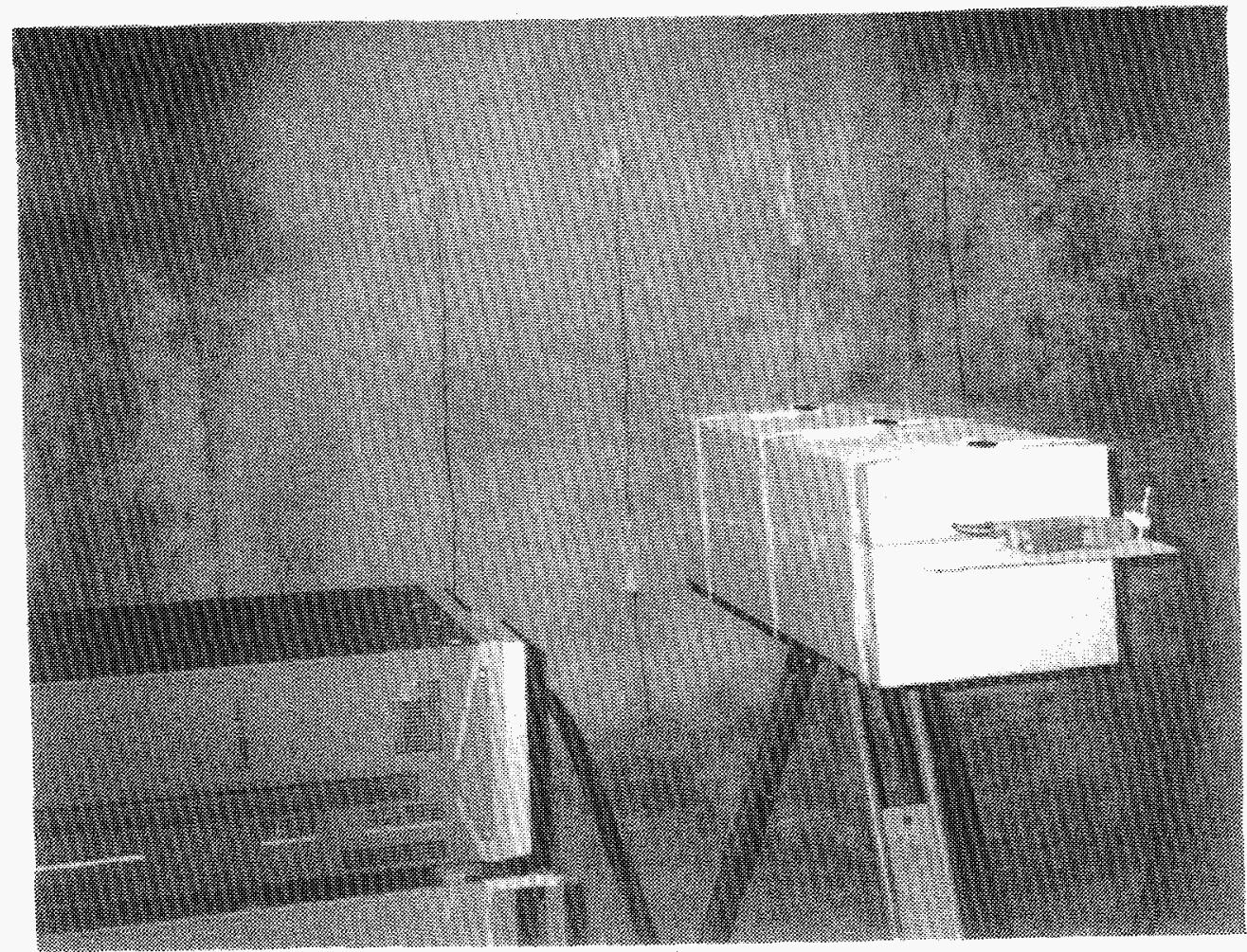

Figure 27. Receiver Setup with Four Vertical Scattering Wires Taped to Concrete Wall at Wright Lab.

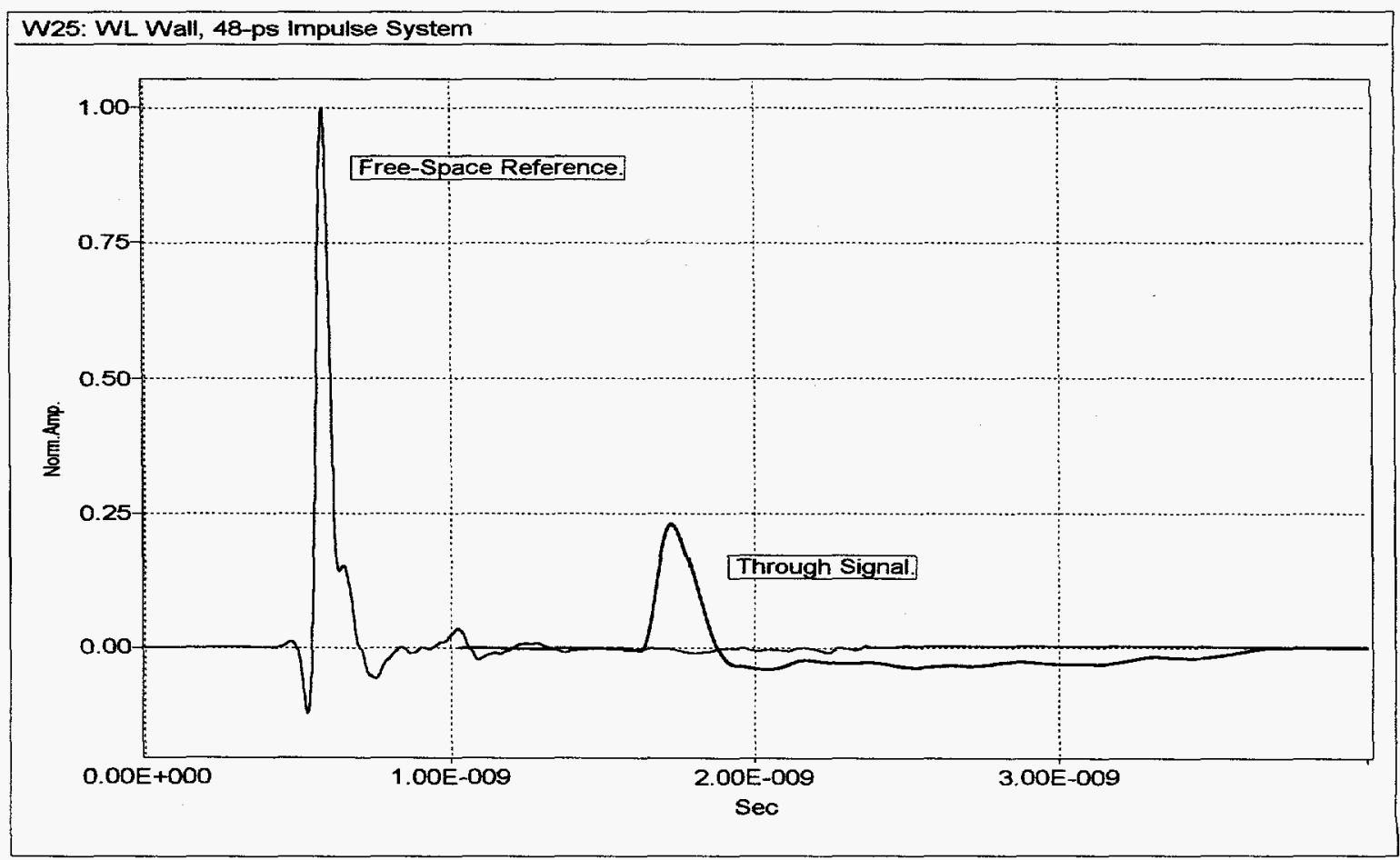

Figure 28. Free-Space and 'Through' Waveforms for Wright Concrete Wall Using Fastest (48-ps) Impulse System; 1 ns/div. 


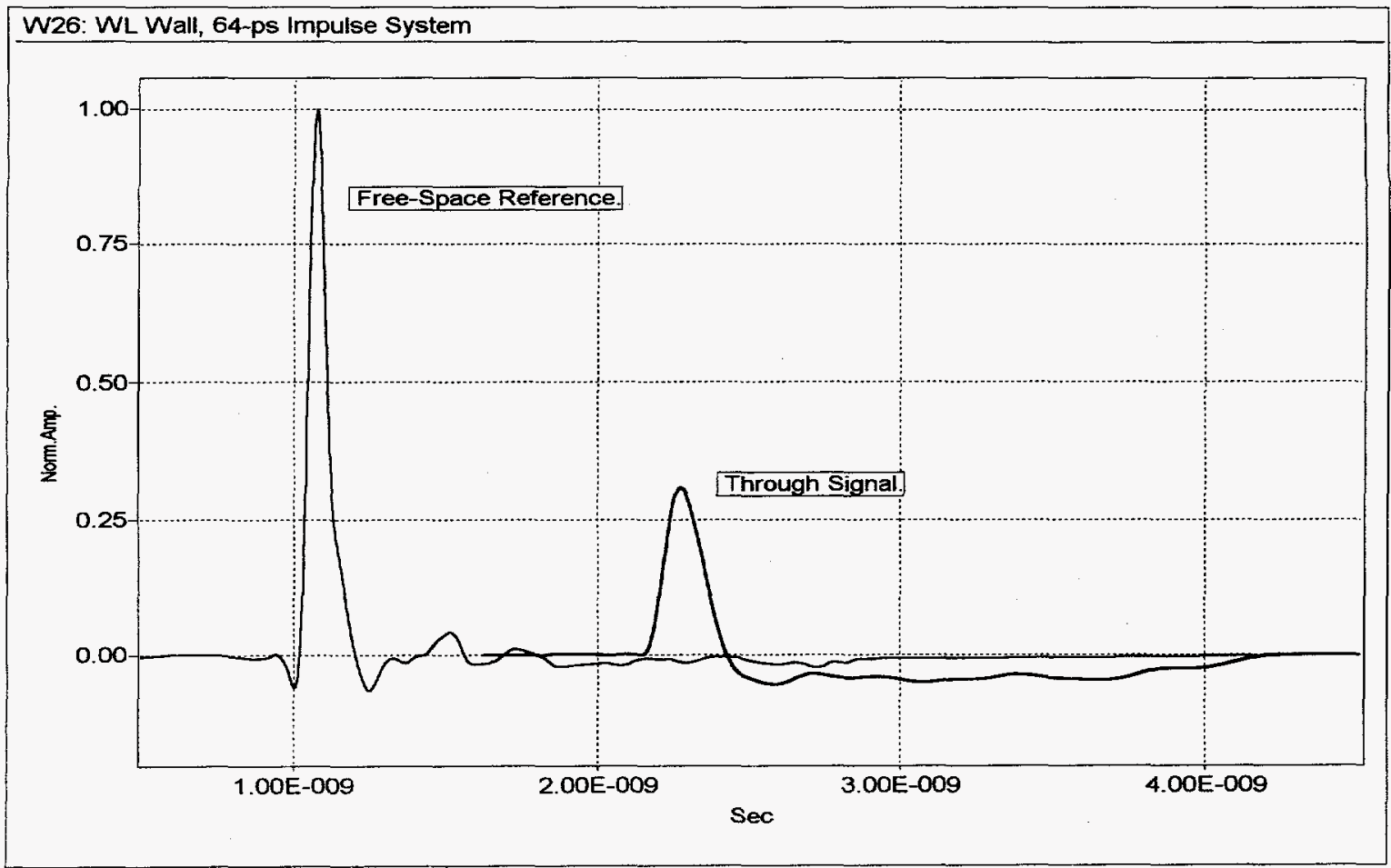

Figure 29. 64-ps Impulse Free-Space and 'Through' Waveforms for Wright Concrete Wall; $1 \mathrm{~ns} /$ div.

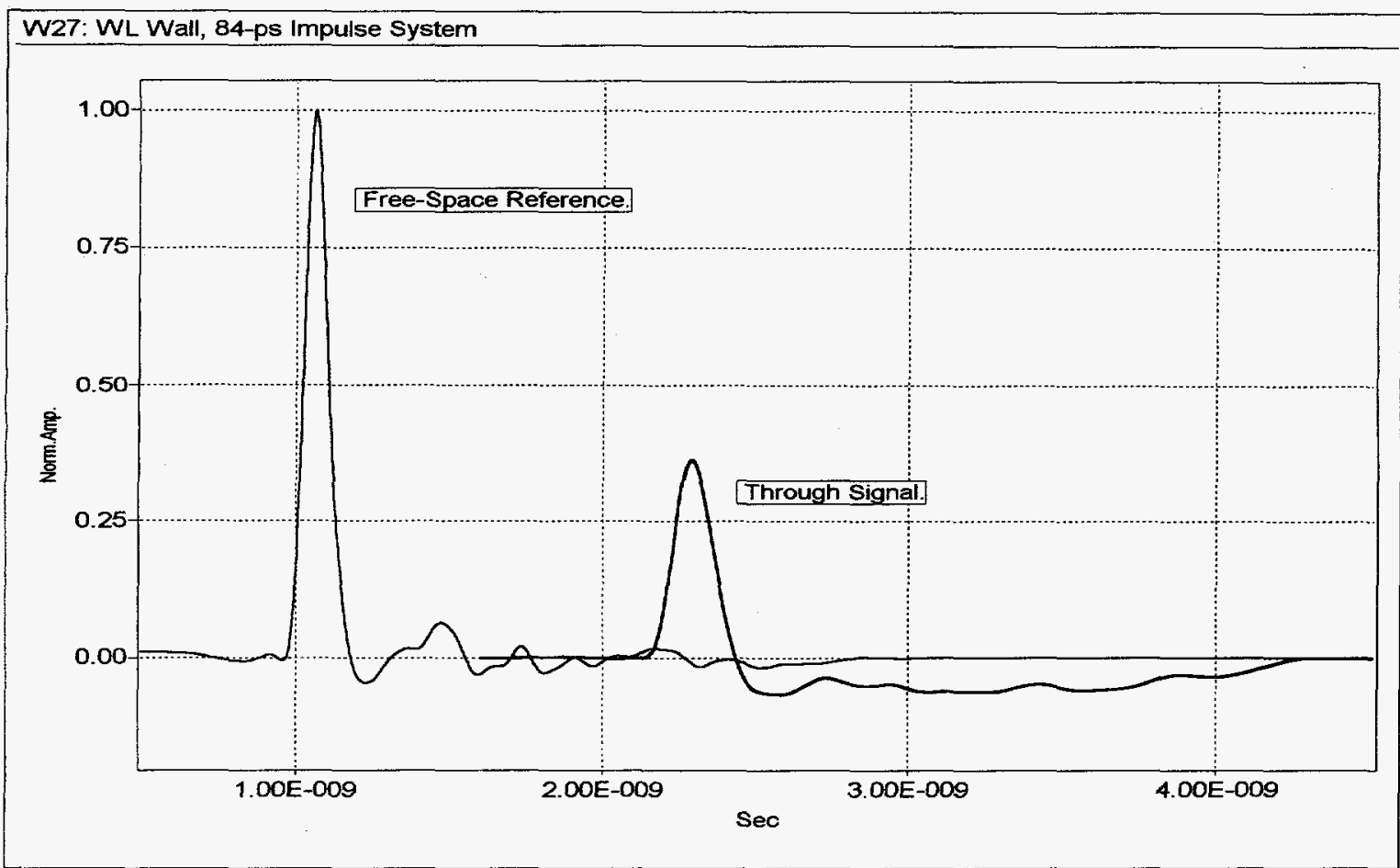

Figure 30. 84-ps Impulse Free-Space and 'Through' Waveforms for Wright Concrete Wall; 1 ns/div. 


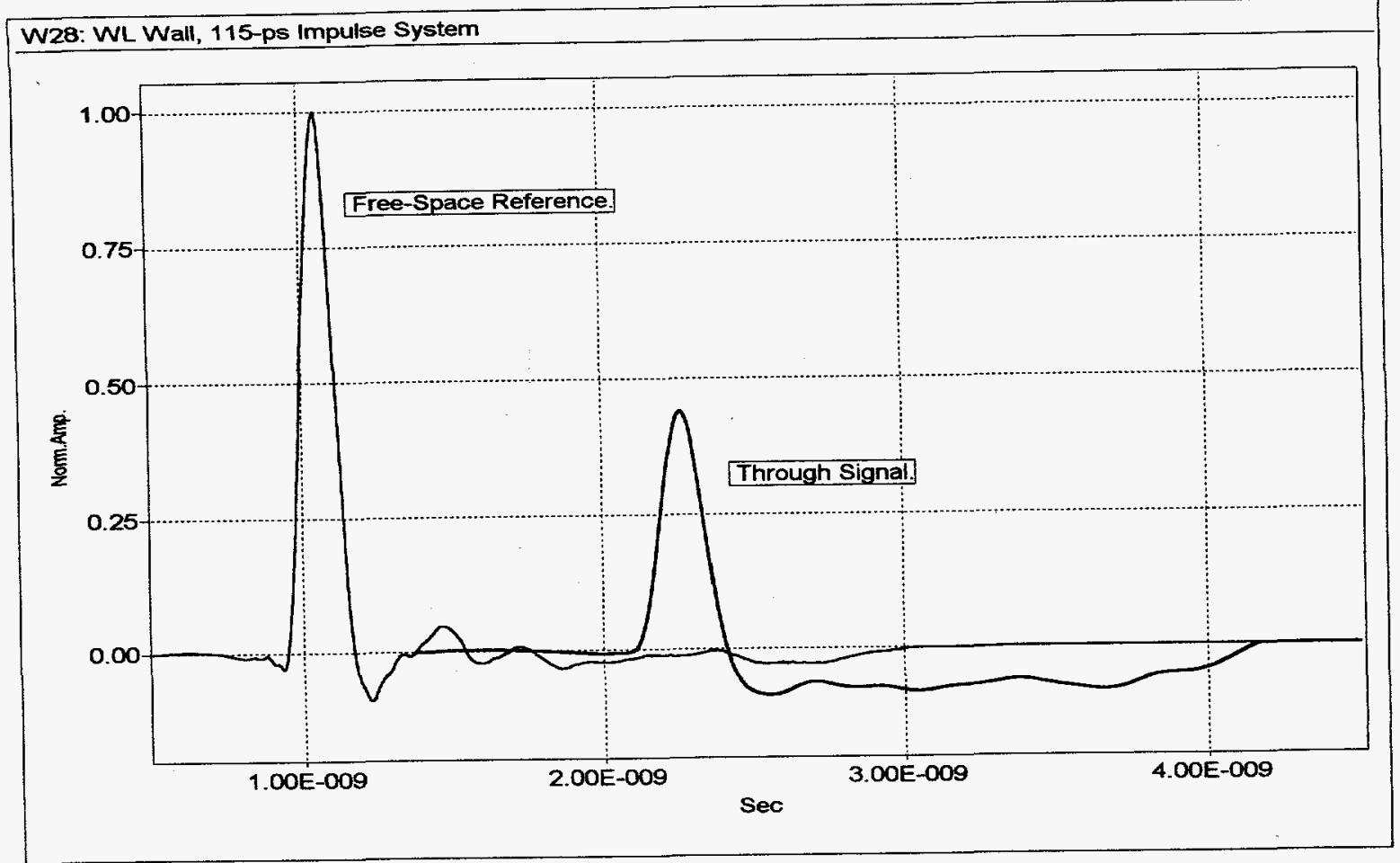

Figure 31. 115-ps Impulse Free-Space and 'Through' Waveforms for Wright Concrete Wall; 1 ns/div.

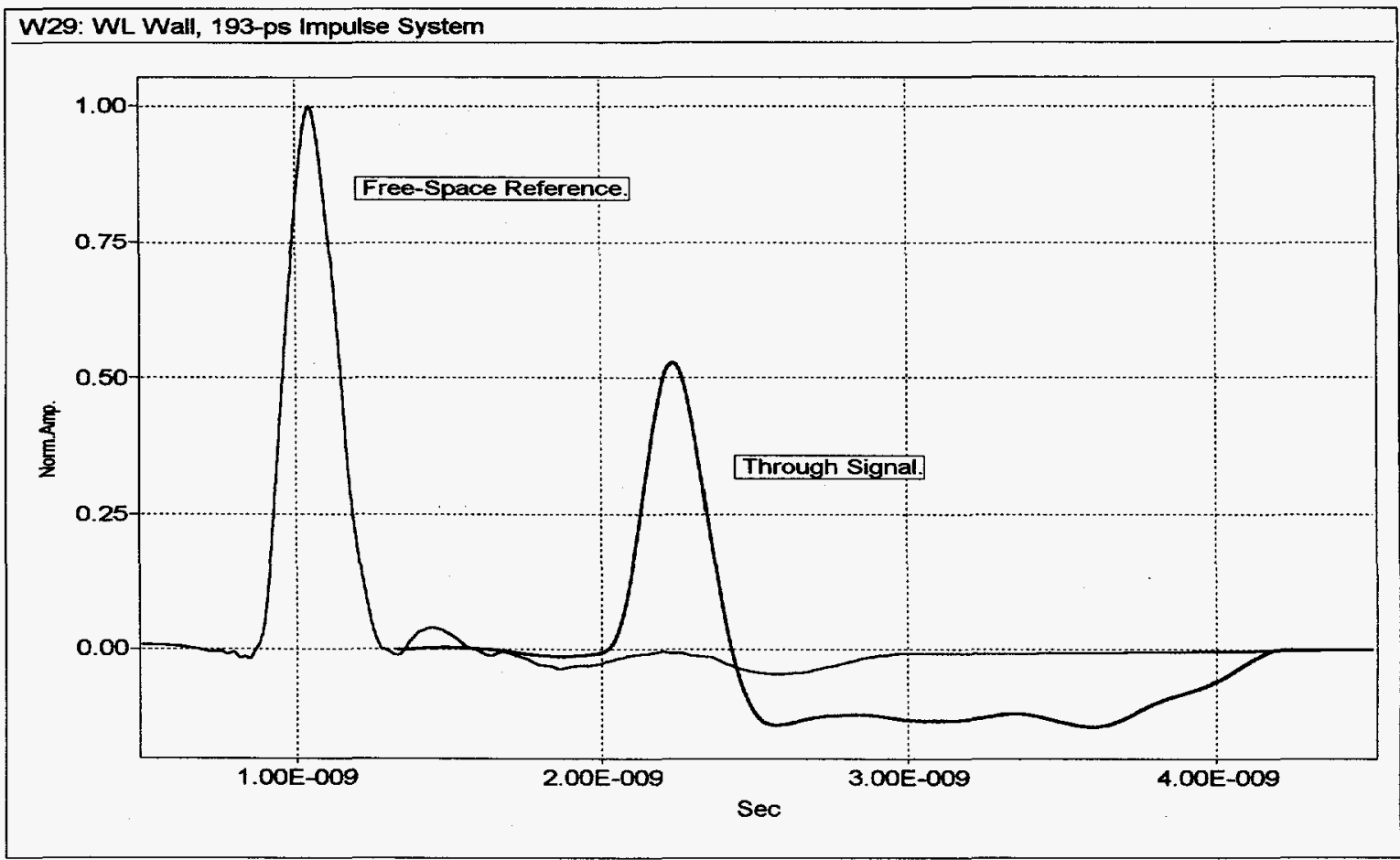

Figure 32. 193-ps Impulse Free-Space and 'Through' Waveforms for Wright Concrete Wall; 1 ns/div. 


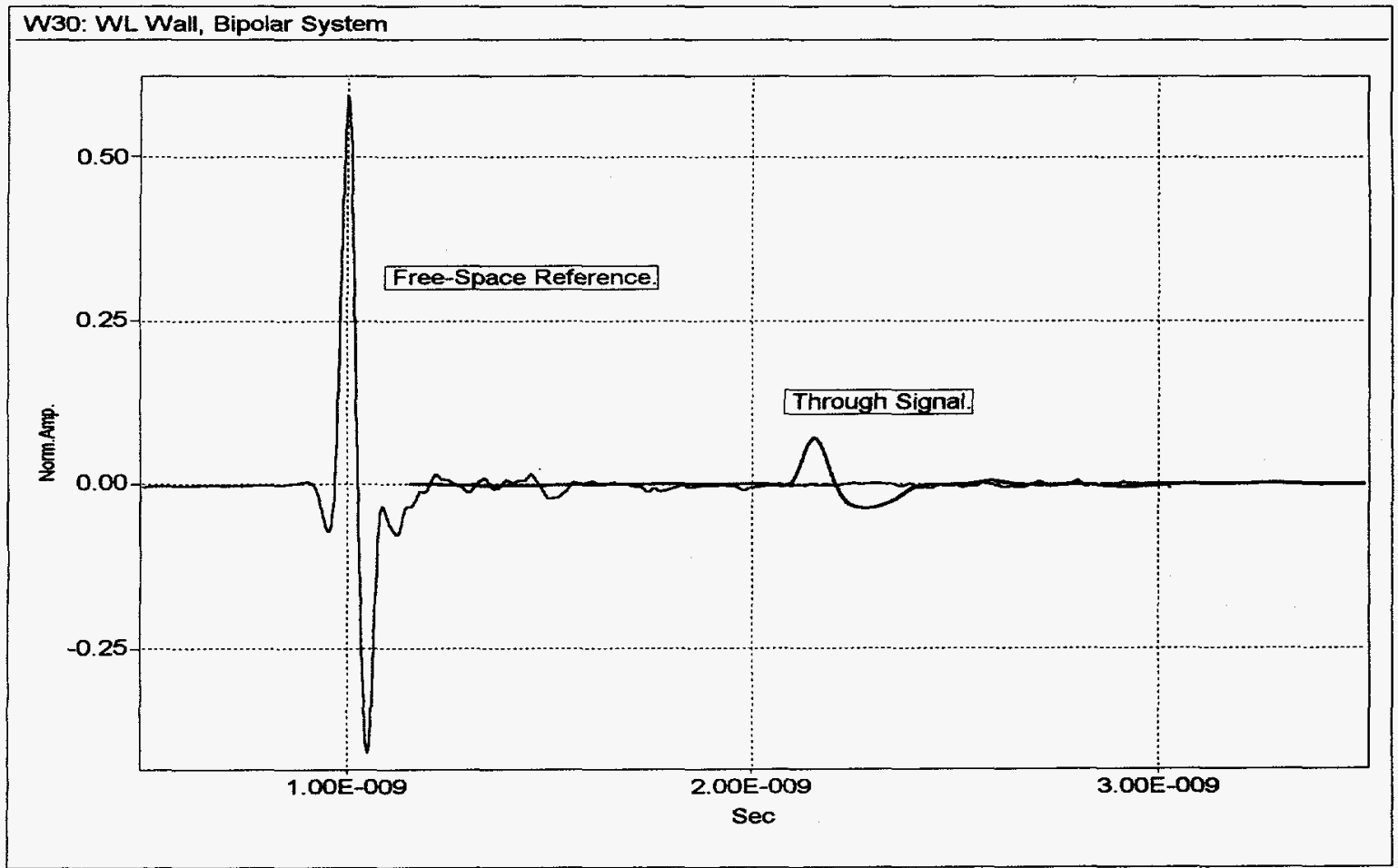

Figure 33. Free-Space and 'Through' Waveforms for Wright Concrete Wall Using Bipolar System; 1 ns/div.

system). These peak-normalized waveforms were processed in the same manner as the Sandia wall data, in order to provide the best (cleanest) characterization of the wall under test. The time delay introduced by the wall is consistent across the set of excitation waveforms, with a mean value of $1.15 \mathrm{~ns}$ for the cross-correlation time lag. Using Equation 13, this implies that the dielectric constant is relatively level (with a value of about 4.5 ) over the spectral content of the excitation pulses.

Figure 34 depicts the transfer function of the Wright wall, derived from the six time-domain datasets above with Equation $9 \mathrm{~b}$. The bold trace is that of the 48-ps impulse system measurement, with the cleanest estimate of $H(f)$ over the widest bandwidth; it begins to get noisy above $14 \mathrm{GHz}$. The bipolar system measurement offered comparable quality in characterization, with the most significant difference occurring in the derived impulse response (to be seen in Figure 35).

For the impulse system measurements, a definite trend was observed as a function of system pulse duration - as the pulse duration increased (slower transient radiated field), the bandwidth of the spectral content of the excitation wave was reduced, resulting in a lower effective bandwidth in the estimated transfer function. The 193-ps measurement yields $H(f)$ good up to $5 \mathrm{GHz}$, above which it is entirely deconvolution noise. In a similar fashion, the 115-ps measurement yields good characterization up to $7 \mathrm{GHz}$, the 84-ps measurement up to $10 \mathrm{GHz}$, and the 64-ps measurement up to about $12 \mathrm{GHz}$. The implication is that the fastest transient measurement system should be used for such propagation experiments, in order to achieve the widest possible 


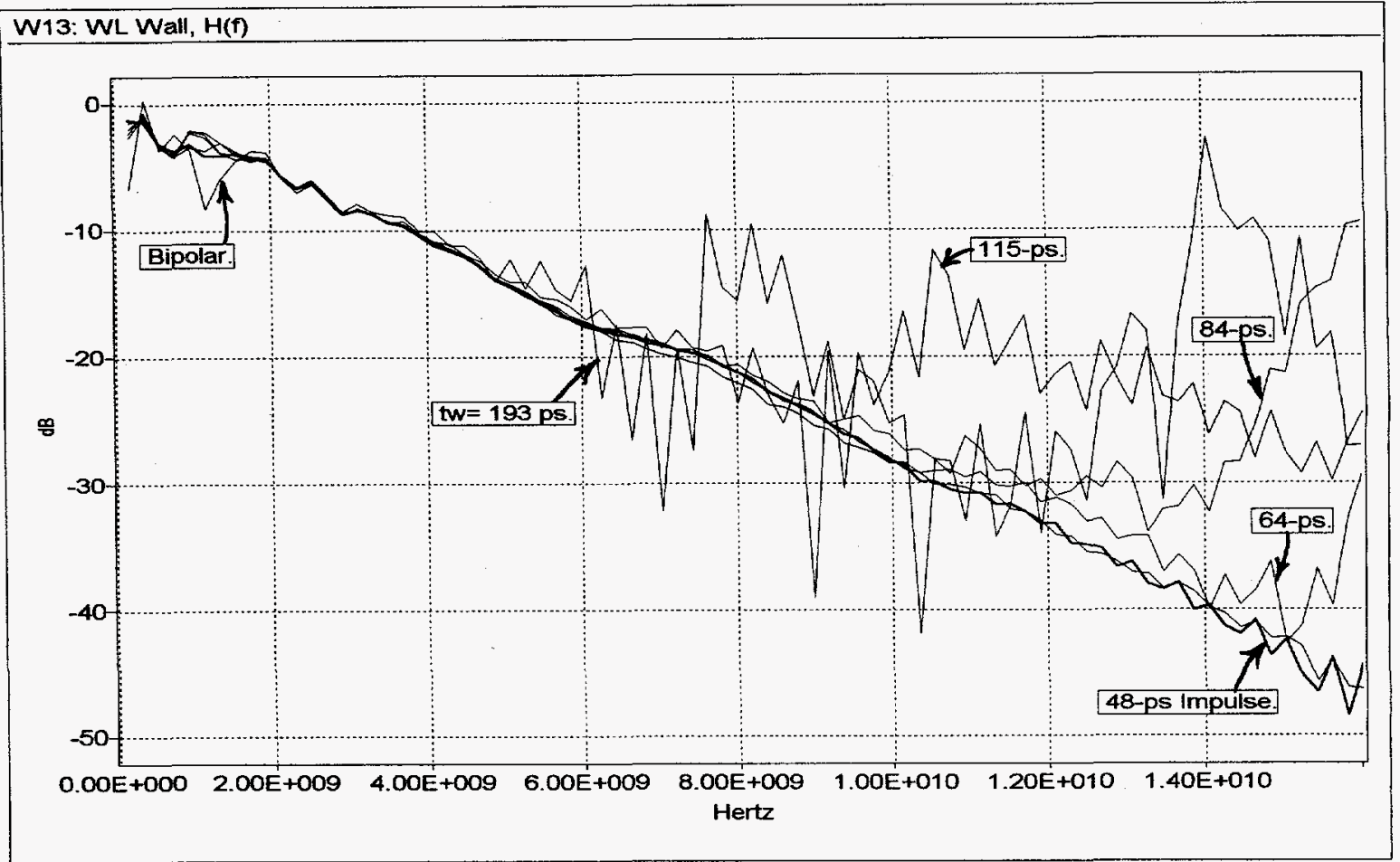

Figure 34. Log-Magnitude of Transfer Function of Wright Concrete Wall Using the Six System Excitation Pulses; 2 GHz/div.

characterization bandwidth. However it should be pointed out that because this propagation phenomenology is like a lowpass filter, a greater fraction of the energy in a transient wave propagates through the walls as the pulse duration slows down. This is easy to see in comparing the relative level of the 'through' waveforms in Figures 28 through 32 as a function of excitation pulse duration. This is entirely explained by the spectral content of the excitation pulse being attenuated by the wall transfer function - the faster the pulse, the higher the spectral content and the more attenuation is incurred by the wave passing through the wall. The percentage of time-domain pulse energy transmitted through the wall was computed versus impulse pulse duration, and it indeed supports this contention; see Figure 50 in the Experimental Summary section.

Figure 35 shows the corresponding impulse response of the Wright wall datasets, computed by the inverse Fourier transform of the transfer function shown in Figure 34. The bold trace is that of the 48-ps impulse system measurement, with the other dataset impulse responses following within $80 \mathrm{ps}$. Note that the late-time negative undershoot of the bipolar system dataset is very different from those of the impulse system datasets. The more pronounced ringing corresponds to the $500-\mathrm{MHz}$ low-frequency peak in $H(f)$ in Figure 34.

Figure 36 shows peak-normalized and time-aligned versions of the above impulse response for the good quality time-domain datasets (the 115-ps and 193-ps impulse system data are not included). The primary impulse response is very consistent between the datasets, with a mean value of $105 \mathrm{ps}$ for the $50 \%$ pulse duration. The negative undershoot features are also correlated 


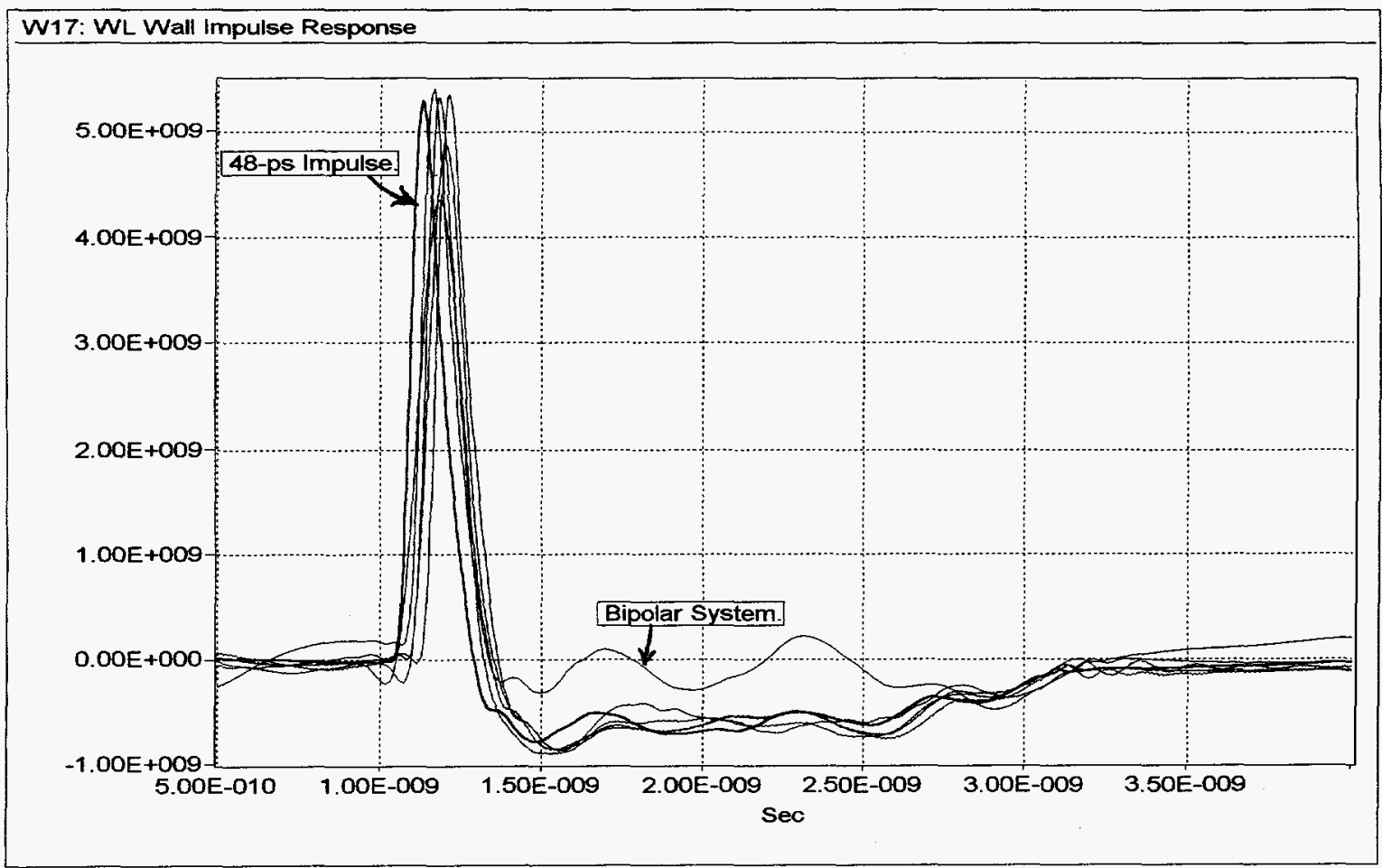

Figure 35. Impulse Response of Wright Concrete Wall Using the Six System Excitations; $500 \mathrm{ps} / \mathrm{div}$.

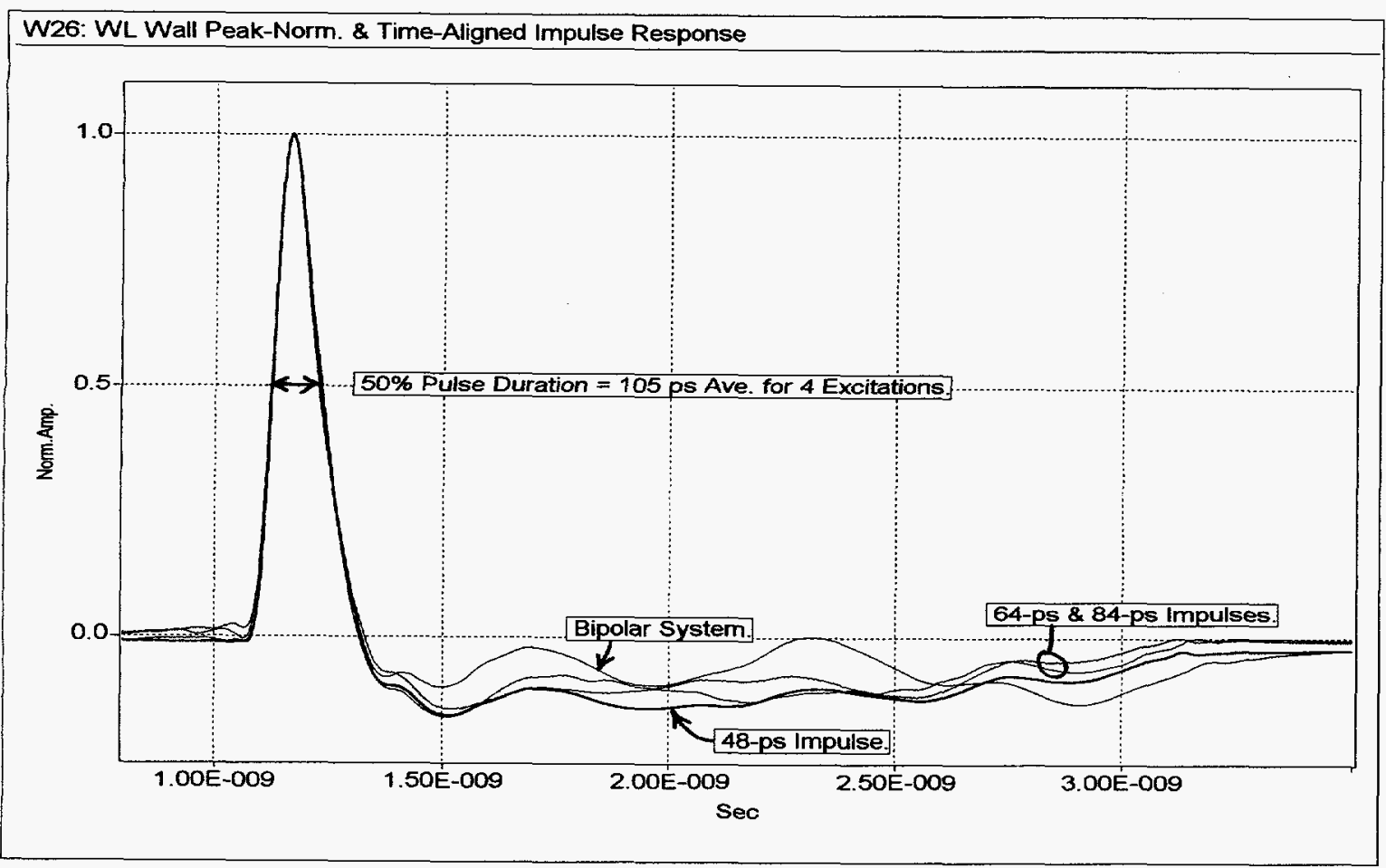

Figure 36. Peak-Normalized and Time-Aligned Impulse Response of Wright Concrete Wall Using 48-ps, 64-ps, and 84-ps Impulses and Bipolar System Excitation; 500 ps/div. 
across the datasets, with the undulations or periodic behavior showing up in differing amounts. As we explained for the Sandia wall experiments, we surmise that these features are in some way associated with wave scattering off the internal vertical rebar. The question remains about why the bipolar pulse yielded the largest difference in characterization compared to the impulse waves.

The other data to show in this section is the derived dielectric constant (Equation 11) of the Wright wall, based on the 48-ps impulse dataset. This is given in Figure 37, along with that of the Sandia wall. Note that the Wright wall dielectric constant is also flat with frequency, and is about $10 \%$ lower, which implies lower moisture content or different long-term curing of the wall. Also like the Sandia wall, this level of dielectric constant (about 4.5) is consistent with the insertion time lag introduced by the wall.

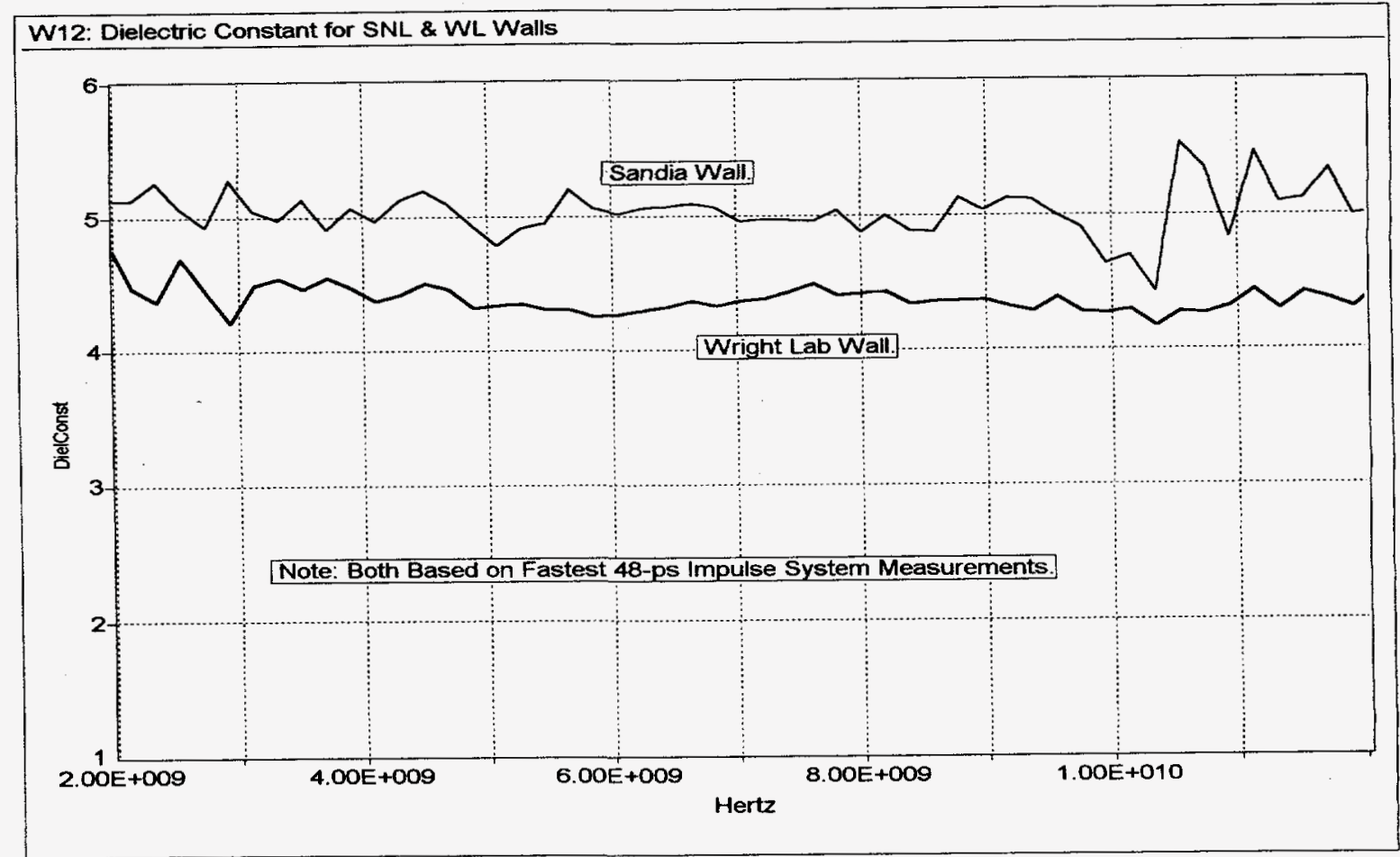

Figure 37. Dielectric Constant of Wright Wall and Sandia Wall, Both Based on the Fastest Impulse Systems. Plotted Over Best Frequency Range, $2 \mathrm{GHz}$ to $12 \mathrm{GHz}$; $1 \mathrm{GHz} / \mathrm{div}$.

In the Experimental Summary section, we will compare the transfer function, impulse response, and attenuation constant for the Sandia and Wright concrete walls.

\section{Wright Lab Sandbox}

We spent over a week performing experiments with the Wright Lab sandbox, which Dr. Min had constructed specifically for propagation studies. These measurements were much more difficult to make than those of the two concrete walls described above, because there is significant diffraction around the sandbox (sides, top, and underneath) which arrives earlier than the direct 'through' wave. This diffracted-wave energy interferes with the direct 'through' energy, making 
it impossible to achieve a clean and unambiguous measurement of the 'through' wave. The sandbox is shown in Figure 38, with the receiving equipment in place; the sandbox was filled with fine white, slightly moist, sand typical of that region in Florida. There are exterior $4 \times 4$ in. vertical wood posts and interior $2 \times 4$ in. vertical posts which support the side walls of $2 \times 12$ in. horizontal wood members (all of the wood is pressure treated). The receive antenna is shown pointed at the fourth section of wall from the left, as defined by the exterior $4 \times 4$ in. uprights. The distance through the 4-cm thick side walls and intervening sand is $1.91 \mathrm{~m}$. For the analysis, we assumed that this entire thickness was composed of the same dielectric constant - we expect that the wood would be similar to the sand, and even if it was significantly different, the proportion of wood relative to the sand is very small, resulting in little error.

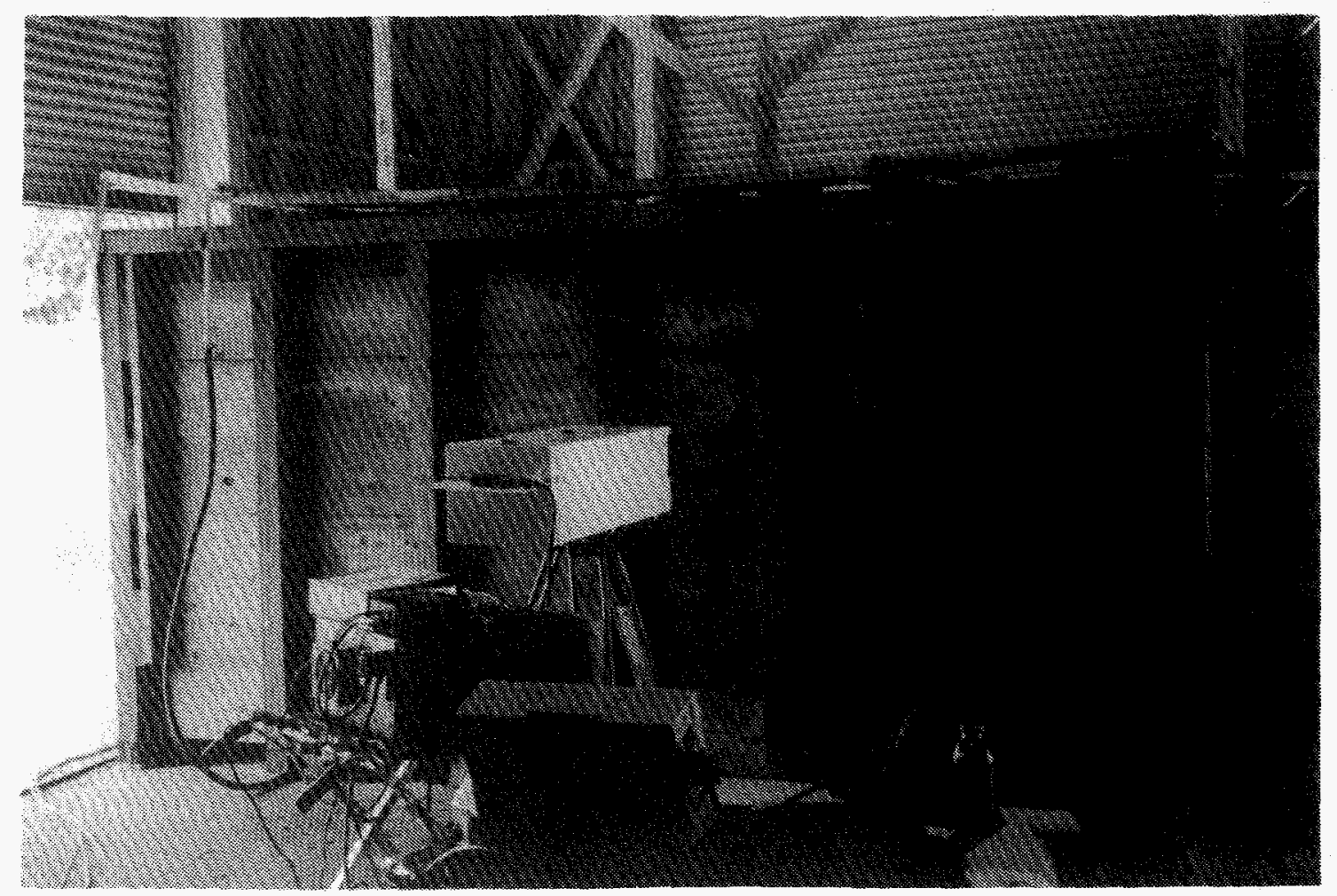

Figure 38. Wright Lab Sandbox, Showing Receiving Side.

We constructed a large wire-mesh diffraction screen flush with the transmitting side wall, $2.4 \mathrm{~m}$ taller than the sandbox and $0.9 \mathrm{~m}$ wider than the sandbox on each side. The wood frame for the diffraction screen can be seen in Figure 39, which shows the final configuration of the receiver for the sandbox 'through' measurements. This wire-mesh screen was only marginally effective in reducing the initial diffraction. Then we assembled simple additional shielding consisting of a 'box' around each antenna; these were made out of double-sided aluminum foil insulation (insulated sheathing for buildings). These shielding boxes helped reduce early diffraction as well, but the lower-frequency diffraction energy could not be totally eliminated. The transmitting setup was similar, on the opposite side of the sandbox.

The walls of the shielding boxes had to be carefully adjusted relative to the antennas. As the shielding walls are brought closer in toward the antennas (a tighter configuration), the diffraction 


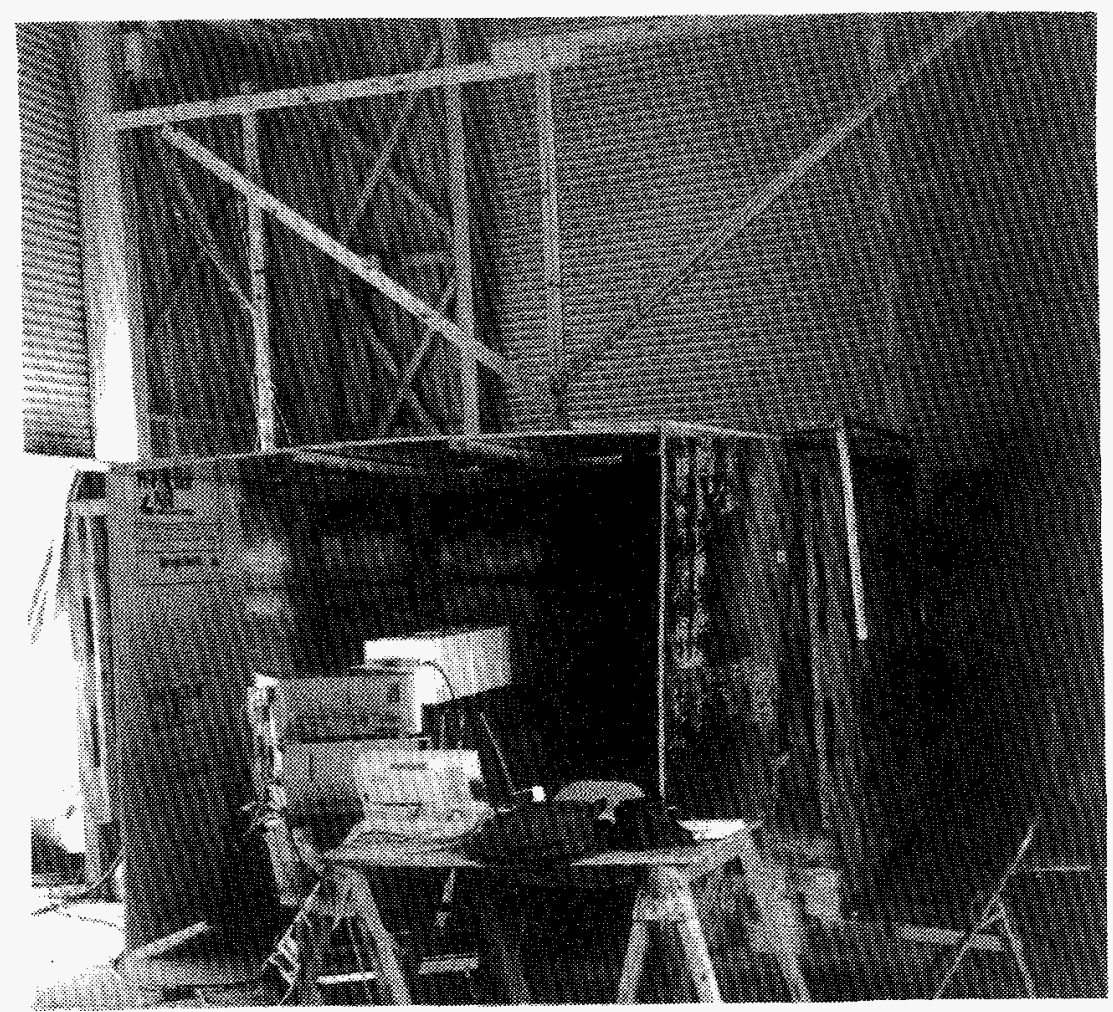

Figure 39. Receiver Setup for 'Through' Measurement of Wright Lab Sandbox.

shielding improves but scattering interference to the direct wave gets worse. If the shielding boxes were too small, then the direct wave component of the received waveform was degraded and reduced. The best configuration of the shielding boxes had side walls positioned $1.0-1.3 \mathrm{~m}$ from the centerline of the antenna and a top wall $0.7-0.9 \mathrm{~m}$ above the propagation centerline.

We spent several days experimenting with the sandbox propagation phenomenology. This included varying the position of the propagation path through the sandbox by moving the pair of antennas horizontally. In addition we dug out sand from the top of the open sandbox, and we also inserted metal rods down into the sandbox at a number of locations between the two side walls. It was straightforward to see the 'through' wave changes created by these perturbations.

We attempted to determine the best experimental configuration for achieving a clean measurement of the direct 'through' wave, but multiple scattering couldn't be avoided. The worst scattering features were due to the closest interior $2 \times 4$ and exterior $4 \times 4$ vertical wood members of the side walls. We even rotated the antennas and measured the horizontally-polarized propagation through the sandbox, but there wasn't a strong direct-wave component like that observed in the usual vertically-polarized measurements. Unfortunately there wasn't enough time to pursue the polarization experiments any further.

After we had determined the best experimental setup for the sandbox measurements, we assembled the corresponding free-space reference setup and acquired the following data. Figure 40 shows the free-space setup, with the transmitter on the left side and the receiver on the right, having an aperture separation of $2.51 \mathrm{~m}$ (corresponding to the sandbox thickness of $1.91 \mathrm{~m}$ 
plus two times a 30-cm antenna-sandbox separation). Note the transverse ground-plane ramp located on the floor between the two antennas. This is a double-sided aluminum sheet like that used for the shielding boxes, partially split in half and folded to form a small diffraction fence. Because the propagation time through the sandbox was $7 \mathrm{~ns}$, it was desirable to acquire the free-space signal with at least that much clear time. However, with an antenna centerline height of $1.21 \mathrm{~m}$ and separation of $2.51 \mathrm{~m}$, the floor bounce occurs only $1.8 \mathrm{~ns}$ after the direct radiated pulse. As a result, we tried several different ground fence configurations in an attempt to minimize the floor bounce component in the received field, and this transverse ramp provided the best reduction.

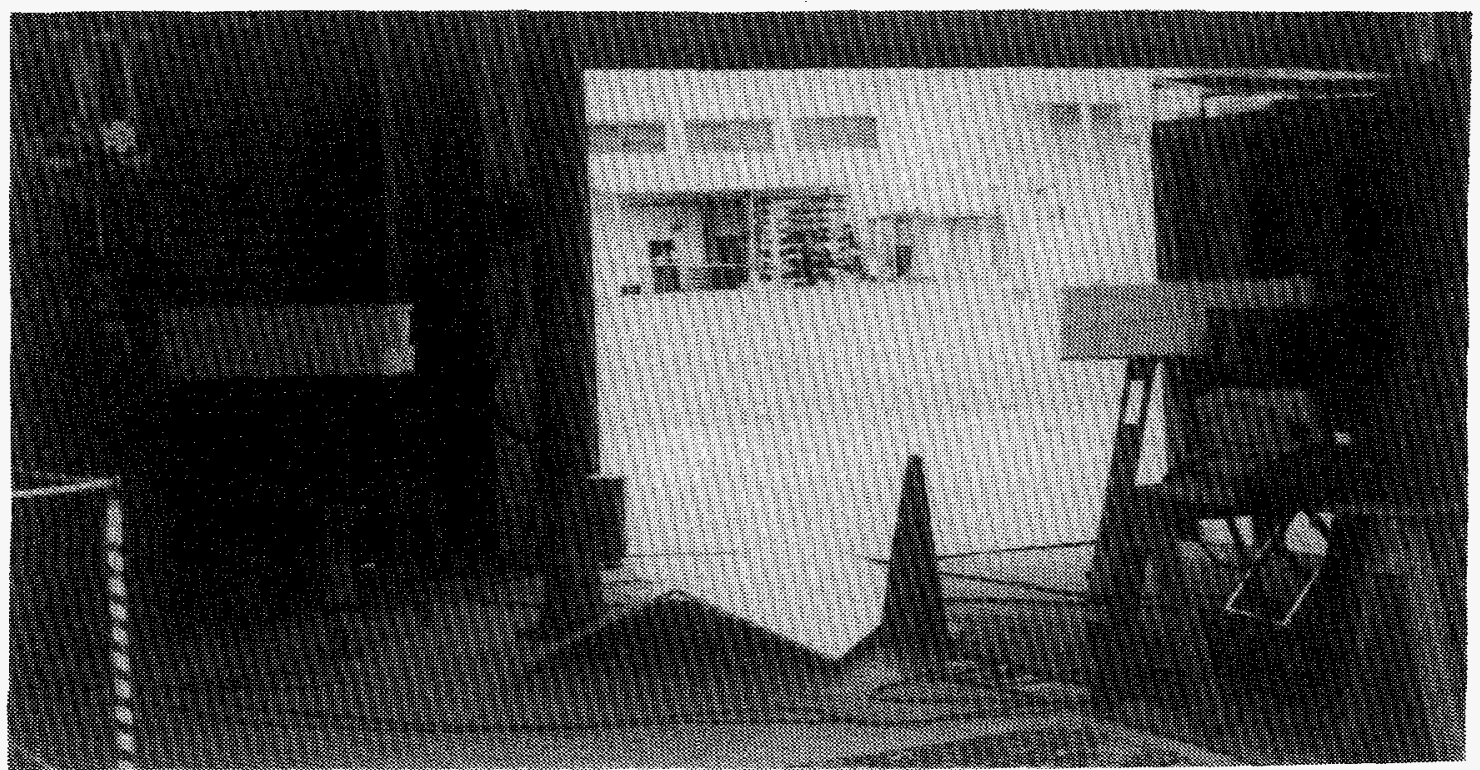

Figure 40. Experimental Setup for Free-Space Measurement of Wright Lab Sandbox.

The free-space and 'through' acquired waveforms are shown in Figure 41, with different time and received-voltage axes (the time axis has the same scale for both traces, but one is shifted). The 'through' waveform is not clean, having two undesired components: early-time external diffraction (around the outside of the sandbox) arriving before the direct-wave component; and late-time interference arriving after the direct wave, caused by continuing external diffraction and internal multiple scattering (of the wave passing through the sandbox). Tic marks delineate the portion of the 'through' waveform which we determined to be the desired single-pass direct component of the wave propagating through the sandbox.

In order to analyze the 'through' signal properly, we extracted the valid portion of the acquired signal, and then performed a curve-fit of the data to produce an extrapolated single-equation model of the direct 'through' wave. This procedure is illustrated in Figure 42, showing a portion of the raw acquired signal, the valid direct-wave part, and the extrapolated modeled signal. TableCurve software (Jandel Scientific) was used for the curve-fitting process, yielding the best approximating function to be the asymmetric-peak beta function. It had the highest correlation coefficient and second-highest F-statistic of all the impulse or peak functions within TableCurve. The received voltage (V) is given by

$$
V_{r}(t)=8.17 \times 10^{-5}-5.3371 \times 10^{11} T^{10.497}(1-T)^{73.019} \text {, }
$$




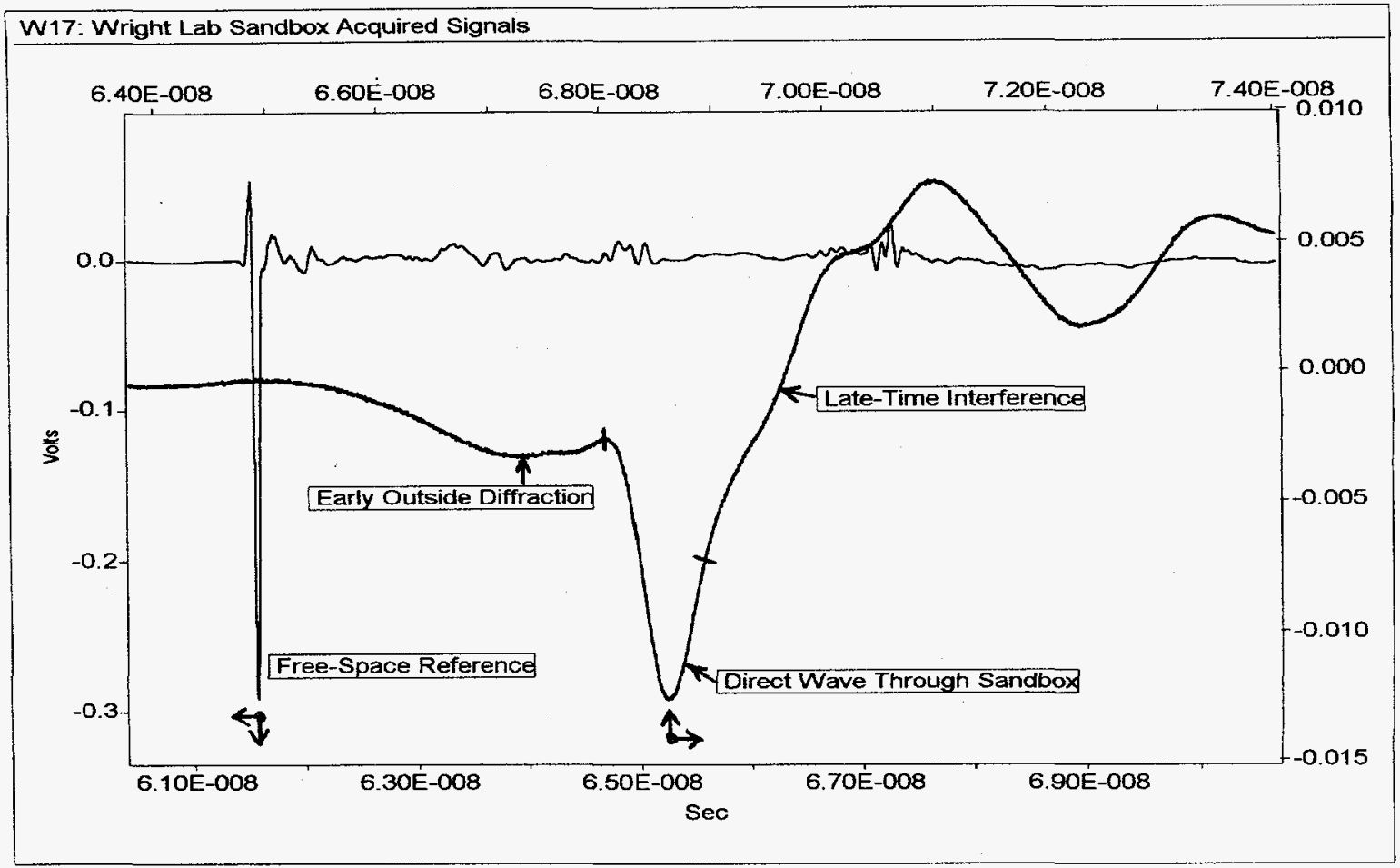

Figure 41. Free-Space and 'Through' Acquired Waveforms for Wright Lab Sandbox; 1 ns/div on Both Traces. Note Significant Diffraction Interference on 'Through' Signal.

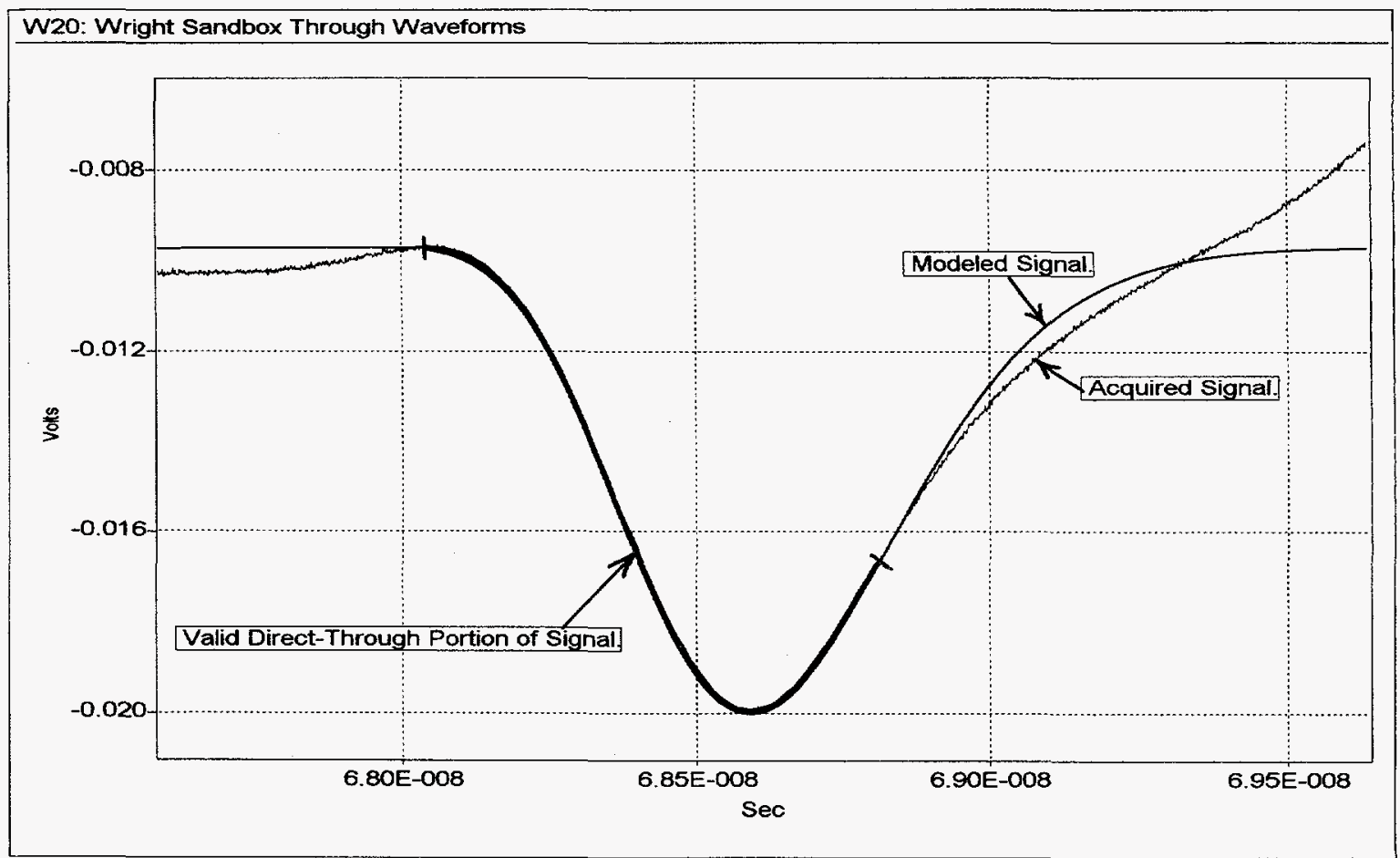

Figure 42. Acquired and Modeled 'Through' Waveforms for Wright Lab Sandbox; $500 \mathrm{ps} / \mathrm{div}$. Bold Portion of Acquired Signal Indicates Valid Direct-Wave Content. 
where $T$ is the normalized time variable (ns) of

$$
T=\frac{t(n s)+0.25292}{6.3962}
$$

This is the equation which was then extrapolated and plotted in Figure 42 as the modeled signal.

Figure 43 shows the resulting processed signals for characterizing the sandbox, both peak-normalized for waveform comparison. The timebase shows the actual time delay of the direct 'through' wave, $7.02 \mathrm{~ns}$ for the peak-peak delay and also the maximum cross-correlation time lag. The modeled 'through' signal had a peak value 28.6 times smaller than that of the system reference signal. This is considerably more attenuation than that imposed by the two concrete walls described earlier, and is primarily due to the sandbox thickness being more than six times greater than that of the walls.

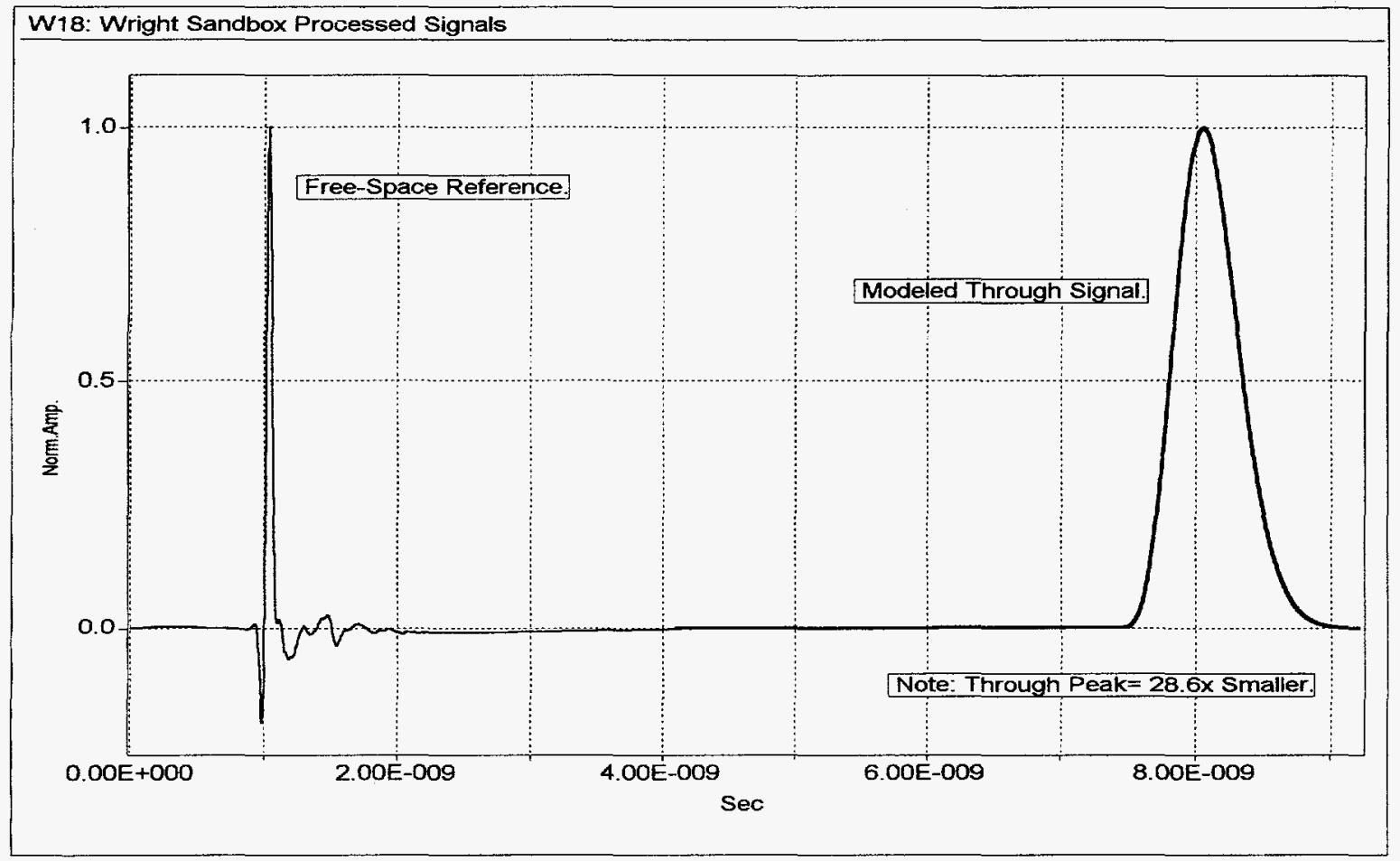

Figure 43. Processed Free-Space and Modeled 'Through' Waveforms for Wright Lab Sandbox; 1 ns/div. Note that Both are Peak-Normalized for Comparison.

Swept-frequency measurements of the sandbox were also performed in order to independently examine the external diffraction and internal scattering interference phenomena. We used the same frequency-domain configuration as that for the Sandia concrete wall, with an HP 8719A vector analyzer covering the $150 \mathrm{MHz}$ to $13.510 \mathrm{GHz}$ range. Figure 44 shows $S_{21}(f)$, the forward scattering parameter between the input port of the transmit antenna and the output port of the receive antenna, without time gating and with time gating to examine only the direct-wave contribution. The bandpass impulse time-domain mode was used to monitor the 'time-domain' view of the swept-frequency data. As the time-domain measurements revealed, there is early-time external diffraction and late-time internal-path multiple scattering occurring. A 2-ns time gate was enabled for the desired direct-wave part of the measurement, and the resulting 
frequency-domain log-magnitude is given in Figure 44. Note the tremendous change due to time gating, with elimination of the lower-frequency multipath energy and an improved higher-frequency noise floor. An important detail on the configuration of the network analyzer is that the minimum window type should be used rather than the normal window type because it introduces the least perturbation of the frequency-domain data at the low end of the sweep. Time gating was also utilized on the free-space system measurement in order to eliminate the error which would be imposed by the floor-bounce energy.

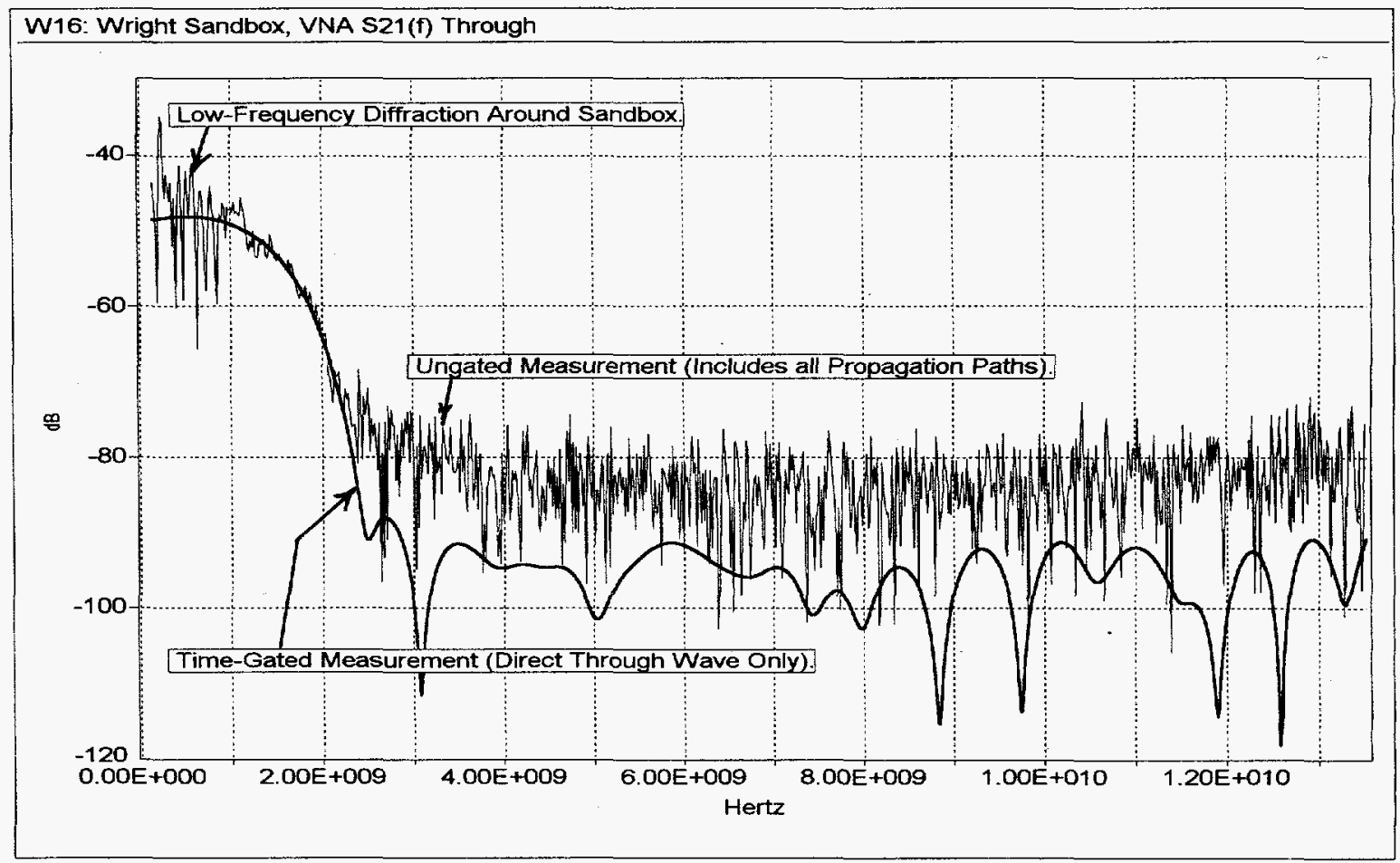

Figure 44. Log-Magnitude of Vector Analyzer 'Through' $S_{21}(f)$ Measurements for Wright Lab Sandbox; Ungated Data and Time-Gated Data; $1 \mathrm{GHz} /$ div.

Based on the time-domain dataset shown in Figure 43, the transfer function of the sandbox was formed (Equation 9b), and is plotted in Figure 45, along with the transfer function based on the time-gated network analyzer dataset. Considering the numerous propagation paths through and around the sandbox, the agreement is very good; also, the swept-frequency dataset validates the modeling effort we applied to the time-domain 'through' signal. The time-domain 'through' data which we acquired has obvious limitations due to the severe interference in the external and internal propagation paths, but our procedure for extracting the desired direct-wave contribution is validated by the network analyzer characterization of the transfer function. For this sandbox, note that the attenuation increases much more rapidly than that of the concrete walls, going into the measurement noise floor below $4 \mathrm{GHz}$.

Then the impulse response of the sandbox is computed from the time-domain dataset transfer function, and is shown in Figure 46. There are two items to notice about this plot. The first is the $50 \%$ pulse duration of $512 \mathrm{ps}$, which is much slower than the 146-ps and 105-ps values for the Sandia and Wright concrete walls respectively; this indicates that much more pulse dispersion 


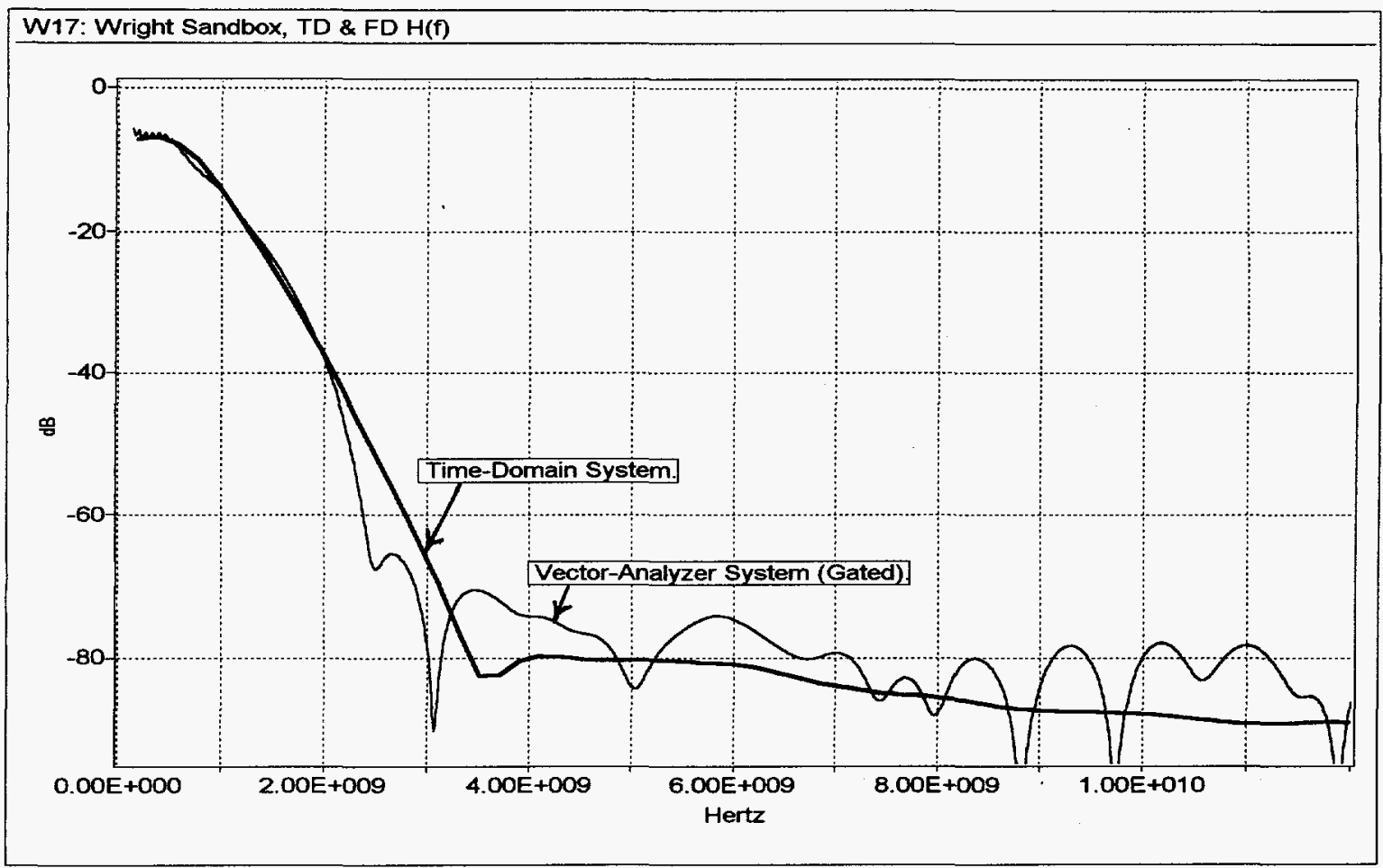

Figure 45. Comparison of Transfer Function Magnitude of Wright Lab Sandbox, for Impulse Time-Domain System and Time-Gated Vector Analyzer System; $1 \mathrm{GHz} / \mathrm{div}$.

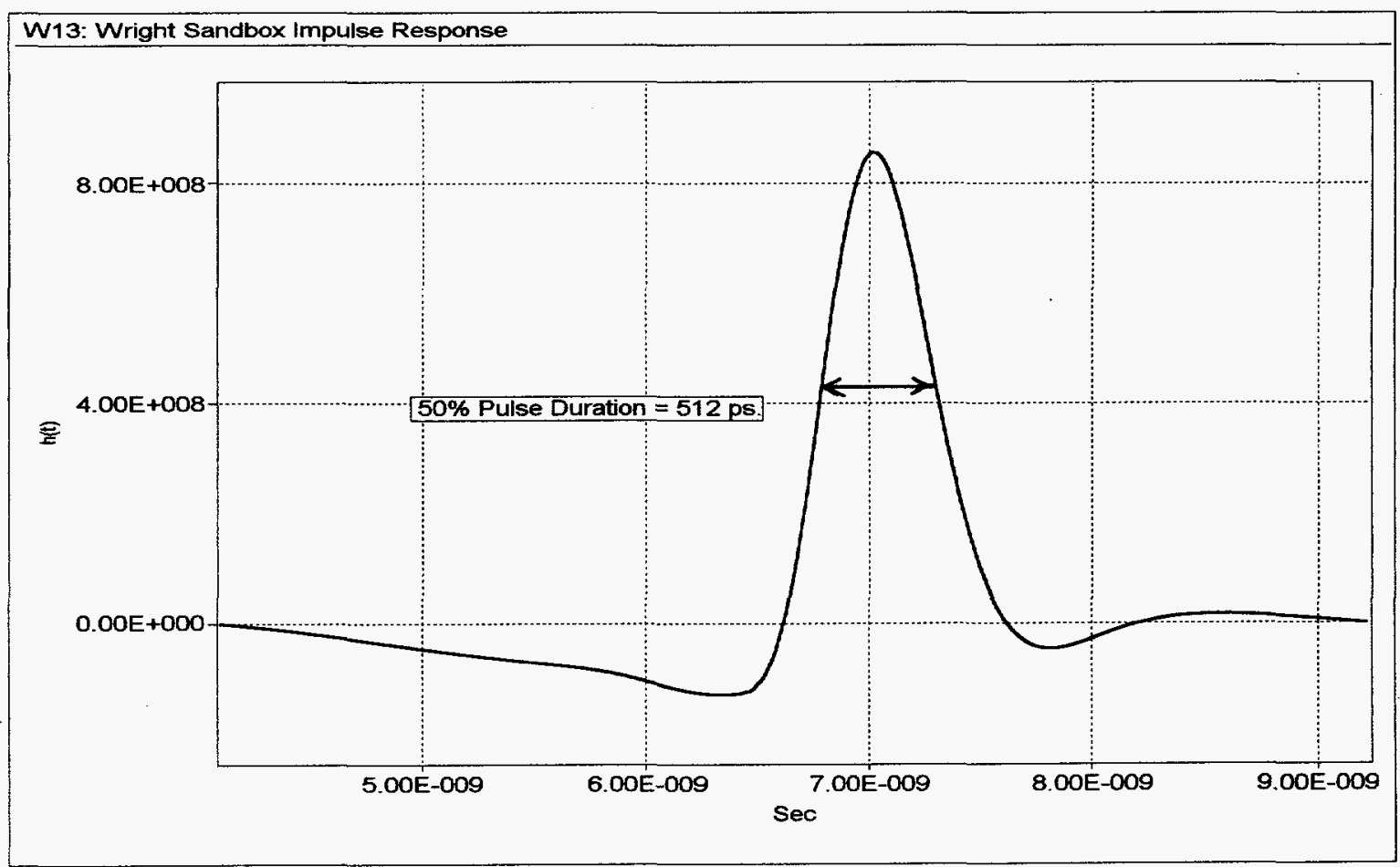

Figure 46. Impulse Response of Wright Lab Sandbox, Based on the Inverse Fourier Transform of the Time-Domain Dataset; 1 ns/div. 
takes place through the thicker sandbox (as expected). The second item to notice is that the insertion time delay is about $7 \mathrm{~ns}$, much longer than that of either concrete wall. This is also due to the increased thickness of the sandbox. It may be pointed out that the leading undershoot should be ignored, because of the limited value of the modeled 'through' data - the model which is assumed for the direct wave will directly affect the resulting transfer function of the sandbox layer under test and the corresponding impulse response.

The attenuation constant of the sandbox will be shown in Figure 49, along with that of the two concrete walls. The dielectric constant versus frequency wasn't worthwhile because the phase of $H(f)$ was not well behaved, due to the errors introduced by the 'through' signal modeling and processing. The cross-correlation time lag yielded a dielectric constant of 4.4 (using Equation 13), which is reasonable for slightly wet silica sand.

\section{Experimental Summary}

During the Series-2 experimental work, we performed very careful propagation measurements through two different 12 -in. thick concrete walls and a $6-\mathrm{ft}$. thick sandbox, utilizing a variety of transient time-domain pulses. The resulting transfer function and corresponding time-domain impulse response were very consistent with each other, and were additionally validated by frequency-domain vector analyzer measurements of the transfer function. This is the first comprehensive data we have seen for very wideband characterization of actual concrete walls (with rebar construction) and sand. Figure 47 shows the log-magnitude of $H(f)$ for the Sandia and Wright walls, based on the fastest time-domain datasets. The Sandia wall has higher loss, but the frequency behavior is obviously the same - gradually increasing insertion loss with frequency. The Wright Lab sandbox exhibited much faster loss versus frequency (as seen in Figure 45) because it was much thicker.

The corresponding impulse response of the two walls is given in Figure 48, based on this transfer function. There are several items to note about these waveforms. One is that the Wright wall has lower pulse attenuation and shorter time delay than the Sandia wall; this implies that the Wright wall has less attenuation and lower dielectric constant than the Sandia wall (evident in the transfer function in Figure 47). Note also that the primary impulse response is replica in nature, and that the average $50 \%$ pulse duration was 105 ps for the Wright wall and 146 ps for the Sandia wall. Thus, the Sandia wall has more time-domain dispersion or pulse spreading than the Wright wall. The last item to notice is the negative undershoot following the impulse portion of the response; as we have explained, we think that this is partially caused or influenced by the reinforcing bar inside the walls. The impulse response of the sandbox (shown in Figure 46) was much slower. The time-domain cross-correlation function shows similar behavior as the derived impulse response, with the same insertion delays and relative pulse attenuation.

The attenuation constant, $\alpha(f)$, is provided in Figure 49 for the two concrete walls and also the Wright Lab sandbox. It represents the volumetric (or bulk) loss of the concrete or sand, with the pulse reflection losses at each layer interface having been removed. It increases monotonically with frequency, and the Sandia wall does indeed have higher loss than the Wright wall, with about 


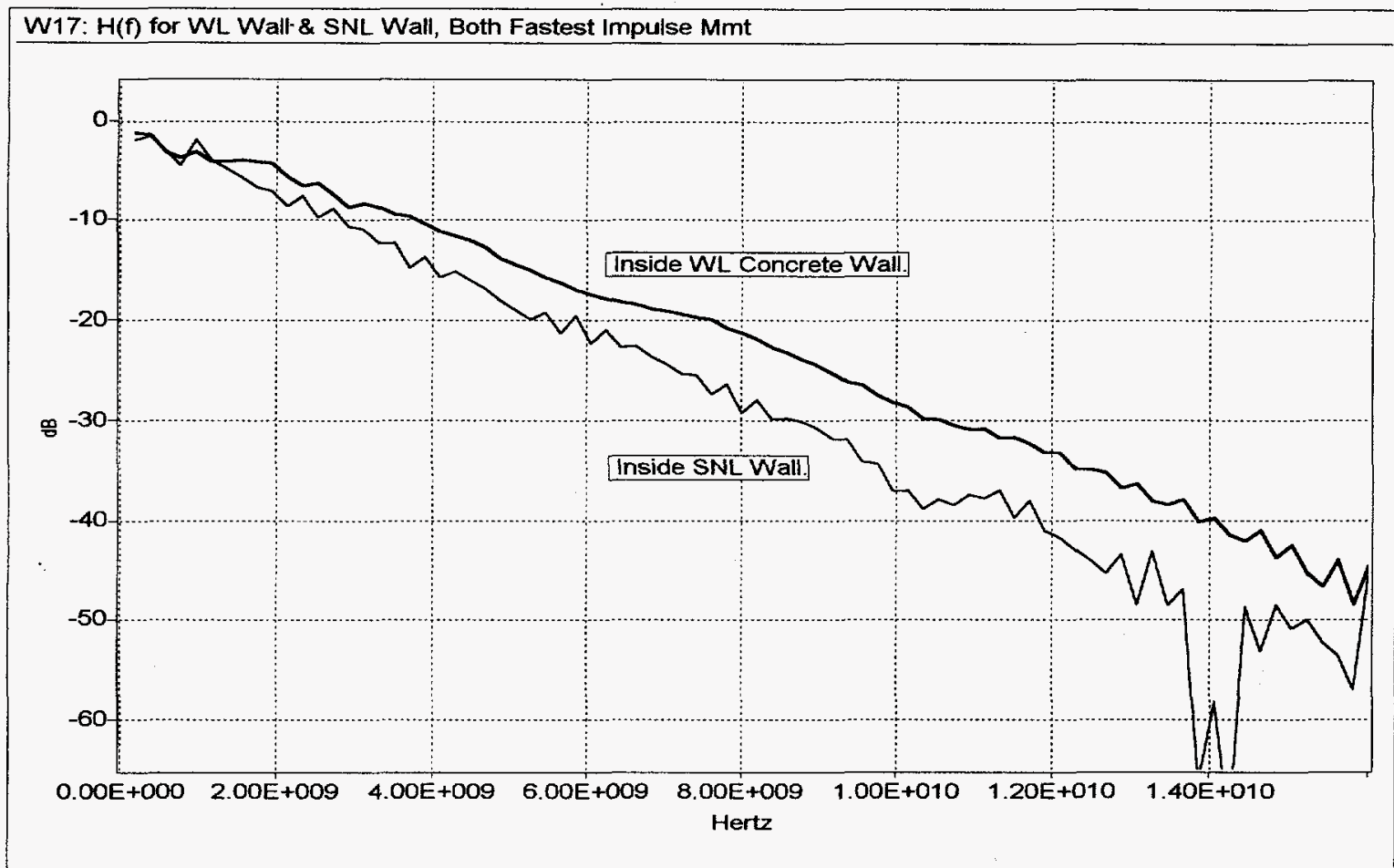

Figure 47. Transfer Function Magnitude for 12-in. Thick Sandia and Wright Concrete Walls, Based on Fastest Impulse Datasets; 2 GHz/div.

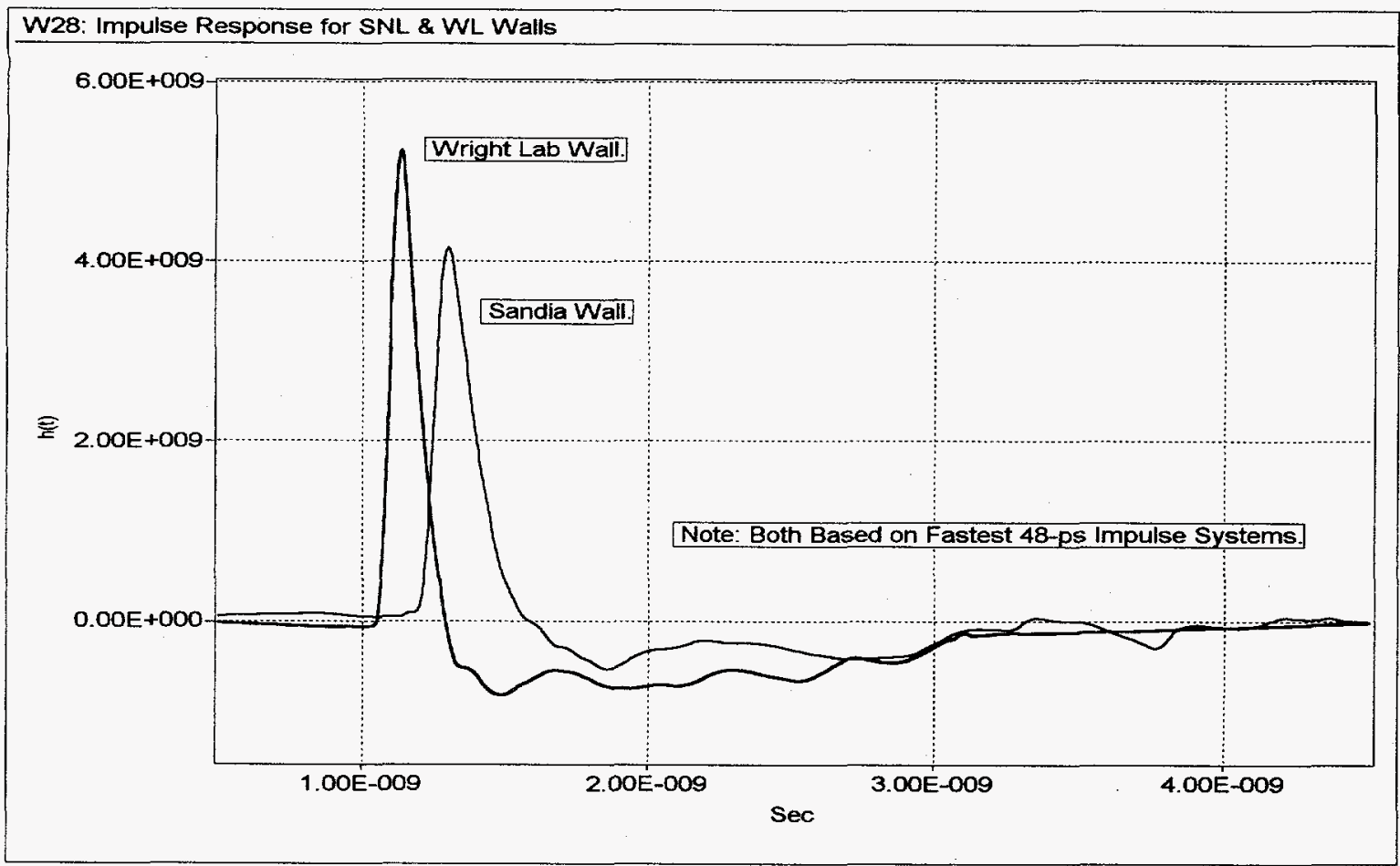

Figure 48. Impulse Response for Sandia and Wright Concrete Walls, Based on Fastest Impulse Datasets; 1 ns/div. 
$20 \mathrm{~dB} / \mathrm{m}$ at $2 \mathrm{GHz}$ and rising to about $110 \mathrm{~dB} / \mathrm{m}$ at $10 \mathrm{GHz}$. The attenuation constant of the sandbox is consistent with that of the walls, but rising at a more rapid rate.

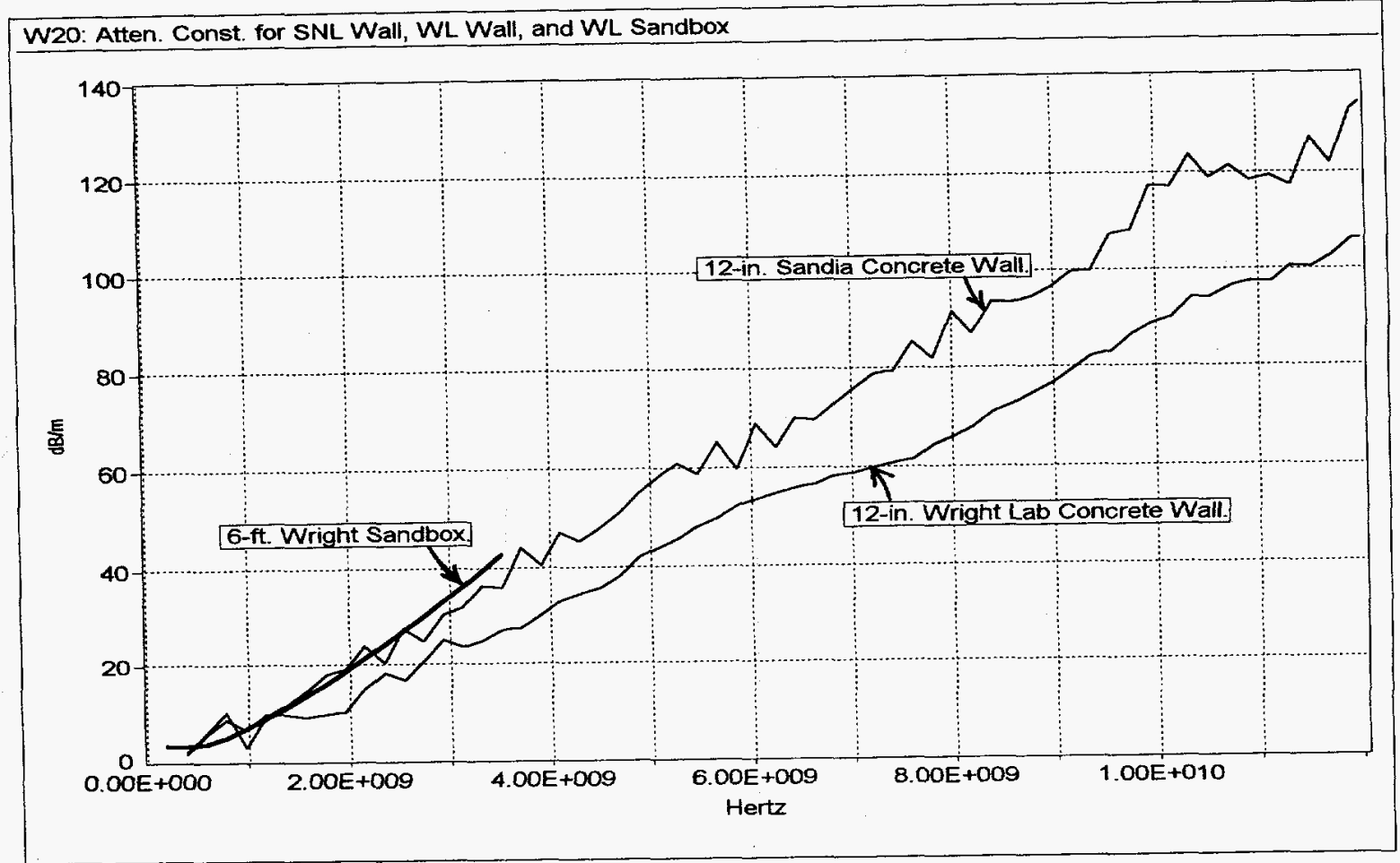

Figure 49. Attenuation Constant $(\mathrm{dB} / \mathrm{m})$ of Sandia and Wright Concrete Walls and Wright Lab Sandbox, Based on Fastest Impulse Datasets; $1 \mathrm{GHz} /$ div.

A time-domain quantification of the transfer function loss through each material is the fraction of pulse energy propagated through the layer. This is plotted in Figure 50 for the two walls, showing the lowpass-filtering trend pointed out earlier - the slower the excitation pulse duration, the less attenuation is experienced by the 'through' wave. The single-pass propagation through the sandbox was $1.4 \%$ for comparison. It should also be pointed out that for the Wright wall, the bipolar pulse experienced greater attenuation than the comparable-bandwidth 48-ps impulse; the transmitted energy was $3 \%$ for the bipolar wave versus $18 \%$ for the impulse wave. This is due to the relative spectral content of the bipolar wave being shifted up in comparison to the spectral content of the impulse wave.

The dielectric constant for the two walls is fairly flat over the UHF to $15-\mathrm{GHz}$ band, consistent with the simple time-delay behavior of the impulse response. The value of dielectric constant based on the maximum cross-correlation time lag (as in Equation 13) was 5.2 for the Sandia wall, 4.5 for the Wright wall, and 4.4 for the Wright Lab sandbox.

The single-pass propagation time-domain behavior has been quantified, and will be very useful in time-domain radar studies for ground-penetrating radar, free-space layered-material measurement systems, etc. Future work could include: time-domain backscatter (radiated reflection) studies to corroborate with the radiated transmission measurement approach; modeling of the data presented herein for system propagation studies; cleaner measurements of soil samples; 


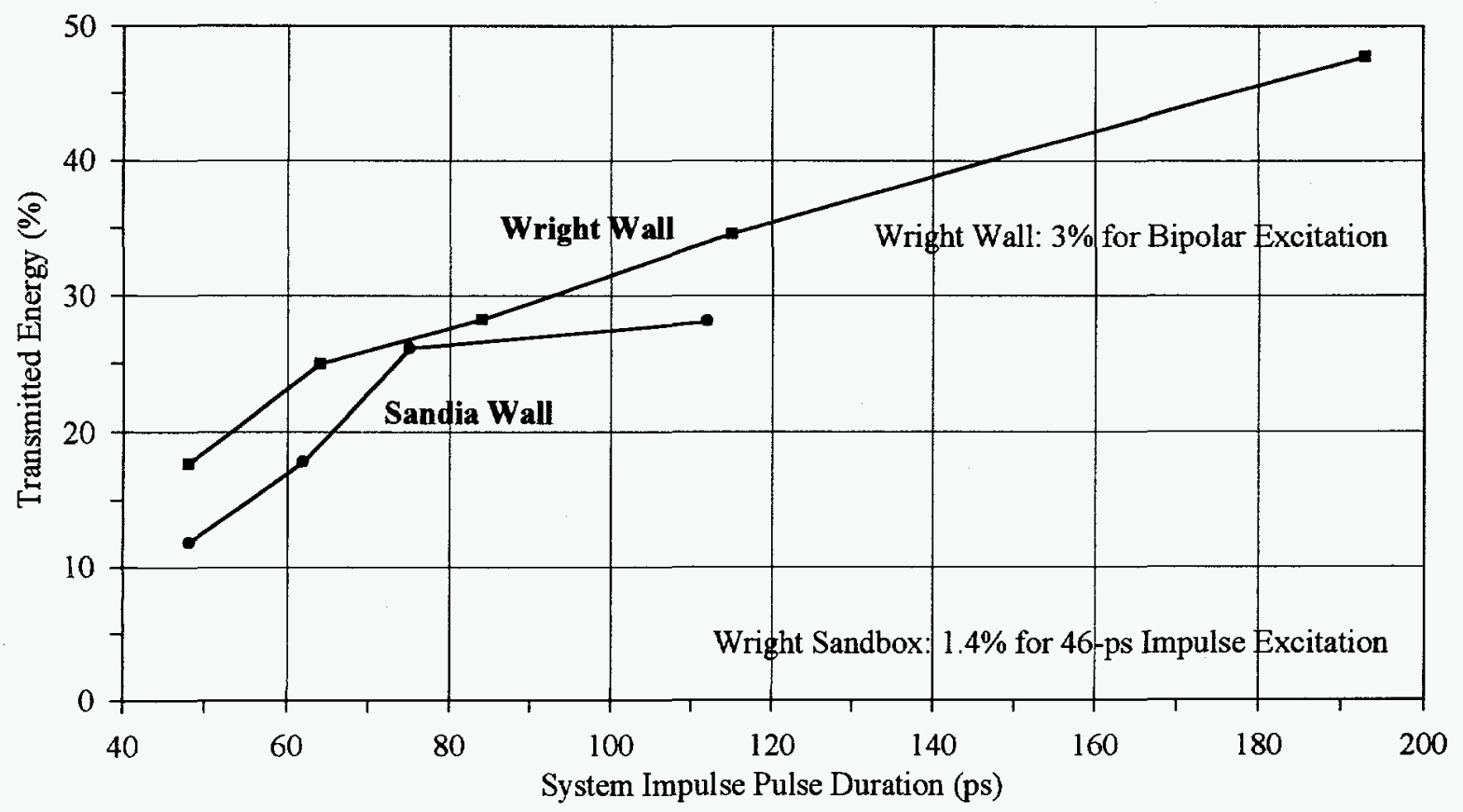

Figure 50. Percentage of Time-Domain Pulse Energy Propagated Through Sandia and Wright Concrete Walls, Based on Impulse Time-Domain Datasets; 20 ps/div.

examination of expected rebar shielding at lower frequencies; and comparison of single-pass transient propagation through layers with sinusoidal steady-state multiple-pass propagation behavior. 
Blank Page 


\section{REFERENCES}

J.R. Andrews, N.S. Nahman, and B.A. Bell, 1982, "Status of Reference Waveform Standards Development at NBS," in Proceedings of the Waveform Recorder Seminar, R.A. Lawton, ed. National Institute of Standards and Technology, Boulder, CO, June, 1992.

G. Arjavalingam, Y. Pastol, J.-M. Halbout, and G.V. Kopcsay, 1990, "Broad-Band Microwave Measurements with Transient Radiation from Optoelectronically Pulsed Antennas," in IEEE Trans. on Microwave Theory and Techniques. Vol. MTT-38(5), May, 1990, pp. 615-621.

J.F. Aurand, 1994, "Time-Domain \& Frequency-Domain Characterization of Two Small, Identical TEM-Horn Antennas." Sandia Abstract SAND93-2251A; presentation at URSI National Radio Science Meeting, Jan. 5-8, 1994, Boulder, CO.

C.A. Balanis, 1989, Chs. 2 and 4 in Advanced Engineering Electromagnetics. Wiley, New York.

J. Barthel, K. Bachhuber, R. Buchner, and H. Hetzenauer, 1990, "Dielectric Spectra of Some Common Solvents in the Microwave Region; Water and Lower Alcohols," in Chemical Physics Letters. Vol. 165(4), 19 January 1990, pp. 369-373.

J.S. Bendat and A.G. Piersol, 1993, Chs. 3 and 6 in Engineering Applications of Correlation and Spectral Analysis, $2^{\text {nd }}$ ed. Wiley, New York.

E.O. Brigham, 1988, Chs. 10 and 14 in The Fast Fourier Transform and its Applications. Prentice-Hall, Englewood Cliffs, NJ.

B.C. Brock and W.E. Patitz, 1993, Optimum Frequency for Subsurface-Imaging Synthetic Aperture Radar. Sandia Report SAND93-0815, May, 1993.

O.M. Bucci, G. Cortucci, G. Franceschetti, C. Savarese, and R. Tiberie, 1972, "Time-Domain Techniques for Measuring the Conductivity and Permittivity Spectrum of Materials," in IEEE Trans. on Instrumentation and Measurement. Vol. IM-21(3), August, 1972, pp. 237-243.

J.-C. Calvet, J.-P. Wigneron, A. Chanzy, S. Raju, and L. Laguerre, 1995, "Microwave Dielectric Properties of a Silt-Loam at High Frequencies," in IEEE Trans. on Geoscience and Remote Sensing. Vol. GE-33(3), May, 1995, pp. 634-641.

J.A. Carpenter, Jr., 1990, "Dielectric Properties Measurements and Data," in Microwave Processing of Materials II. Symposium Proceedings Vol. 189, pp. 477-487, Materials Research Society, Pittsburgh, PA.

R.S. Clark, L.F. Rinehart, M.T. Buttram, and J.F. Aurand, 1993, "An Overview of Sandia National Laboratories' Plasma Switched, Gigawatt, Ultra-Wideband Impulse Transmitter Program," in Ultra-Wideband, Short-Pulse Electromagnetics. Pp. 93-98, Proceedings of 
International Conference on Ultra-Wideband, Short-Pulse Electromagnetics, Oct. 8-10, 1992. Plenum Press, New York.

G.G. Clemeña, 1991, "Short-Pulse Radar Methods," Ch. 11 in CRC Handbook on Nondestructive Testing of Concrete, V.M. Malhotra and N.J. Carino, eds. CRC Press, Boca Raton, FL.

R.E. Collin, 1992, Chs. 2 and 3 in Foundations for Microwave Engineering, $2^{\text {nd }}$ ed. McGraw-Hill, New York.

M.C. Dobson, F.T. Ulaby, M.T. Hallikainen, and M.A. El-Rayes, 1985, "Microwave Dielectric Behavior of Wet Soil - Part II: Dielectric Mixing Models," in IEEE Trans. on Geoscience and Remote Sensing. Vol. GE-23(1), January, 1985, pp. 35-46.

J.A. Fuller and J.R. Wait, 1976, "A Pulsed Dipole in the Earth," Ch. 5 in Transient Electromagnetic Fields, L.B. Felsen, ed. Springer-Verlag, New York.

W.L. Gans, R.G. Geyer, and W.K. Klemperer, 1991, Quantifying Standard Performance of Electromagnetic-Based Mine Detectors. Report NISTIR 3982, National Institute of Standards and Technology, Boulder, CO, October, 1991.

D.K. Ghodgaonkar, V.V. Varadan, and V.K. Varadan, 1989, "A Free-Space Method for Measurement of Dielectric Constants and Loss Tangents at Microwave Frequencies," in IEEE Trans. on Instrumentation and Measurement. Vol. IM-37(3), June, 1989, pp. 789-793.

M.T. Hallikainen, F.T. Ulaby, M.C. Dobson, M.A. El-Rayes, and L.-K. Wu, 1985, "Microwave Dielectric Behavior of Wet Soil - Part I: Empirical Models and Experimental Observations," in IEEE Trans. on Geoscience and Remote Sensing. Vol. GE-23(1), January, 1985, pp. 25-34.

C.D. Hechtman and C.-W. Hsue, 1989, "Transient Analysis of a Step Wave Propagating in a Lossy Dielectric," in Journal of Applied Physics. Vol. 65(9), 1 May 1989, pp. 3335-3339.

C.D. Hechtman and C.-W. Hsue, 1990, "Time-domain Scattering Parameters for a Lossy Dielectric," in Journal of Applied Physics. Vol. 67(5), 1 March 1990, pp. 2199-2209.

J.E. Hipp, 1974, "Soil Electromagnetic Parameters as Functions of Frequency, Soil Density, and Soil Moisture," in Proceedings of the IEEE. Vol. 62(1), January, 1974, pp. 98-103.

P. Hoekstra and A. Delaney, 1974, "Dielectric Properties of Soils at UHF and Microwave Frequencies," in Journal of Geophysical Research. Vol. 79(11), April 10, 1974, pp. 1699-1708.

W. Honcharenko, H.L. Bertoni, and J. Dailing, 1994, "Mechanisms Governing Wireless Propagation Between Different Floors in Buildings," in Microwave Journal. Vol. 37(2), February, 1994, pp. 24-34. 
J. Horikoshi, K. Tanaka, and T. Morinaga, 1986, “1.2 GHz Band Wave Propagation Measurements in Concrete Building for Indoor Radio Communications," in IEEE Transactions on Vehicular Technology. Vol. VT-35(4), November, 1986, pp. 146-152.

R.W.P. King and G.S. Smith, 1981, Antennas in Matter (Fundamentals, Theory, and Applications). MIT Press, Cambridge, MA

J.-F. Lafortune and M. Lecours, 1990, "Measurement and Modeling of Propagation Losses in a Building at $900 \mathrm{MHz}$," in IEEE Transactions on Vehicular Technology. Vol. VT-39(2), May, 1990, pp. 101-108.

R.A. Lawton and A.R. Ondrejka, 1978, Antennas and the Associated Time Domain Range for the Measurement of Impulsive Fields. NBS Technical Note 1008, now the National Institute of Standards and Technology, Boulder, CO, November, 1978.

M. Maurens, A. Priou, P. Brunier, S. Aussudre, M. Lopez, and P. Combes, 1992, "Free-Space Microwave Measurement Technique for Composite Materials," Ch. 11 in Dielectric Properties of Heterogeneous Materials, A. Priou, ed. PIER 6, Elsevier, New York.

E.K. Miller, ed., 1986, Chs. 1, 9, and 10 in Time-Domain Measurements in Electromagnetics. Van Nostrand Reinhold, New York.

J. Musil and F. Zacek, 1986, Microwave Measurements of Complex Permittivity by Free Space Methods and Their Applications. Elsevier, New York.

E. Nyfors and P. Vainikainen, 1989, Industrial Microwave Sensors. Artech House, Norwood, MA.

A.R. Ondrejka, J.M. Ladbury, and H.W. Medley, TEM Horn Antenna Design Guide.

Unpublished Technical Report, National Institute of Standards and Technology, Boulder, CO.

K.E. Oughstun and G.C. Sherman, 1994, Electromagnetic Pulse Propagation in Causal Dielectrics. Springer-Verlag, New York.

S.J. Patsiokas, B.K. Johnson, and J.L. Dailing, 1986, "Propagation of Radio Signals Inside Buildings at 150, 450, and $850 \mathrm{MHz}$," in Proceedings of IEEE 36th Vehicular Tech. Conference. Pp. 66-71, IEEE Press, New York.

N.R. Peplinski, F.T. Ulaby, and M.C. Dobson, 1995, "Dielectric Properties of Soils in the 0.3-1.3-GHz Range," in IEEE Trans. on Geoscience and Remote Sensing. Vol. GE-33(3), May, 1995, pp. 803-807. Corrections in Vol. GE-33(6), November, 1995, p. 1340.

D.M. Pozar, 1990, Ch. 2 in Microwave Engineering. Addison-Wesley, Reading, MA. 
S. Ramo, J.R. Whinnery, and T. Van Duzer, 1965, Ch. 6 in Fields and Waves in Communication Electronics. Wiley, New York.

G.C. Rose, R.J. Churchill, and K.R. Cook, 1972, "Determination of Complex Dielectric Constant of High-Loss Materials," in IEEE Trans. on Instrumentation and Measurement. Vol. IM-21(3), August, 1972, pp. 286-287.

K. Sato, T. Manabe, J. Polivka, T. Ihara, Y. Kasashima, and K. Yamaki, 1996, "Measurement of the Complex Refractive Index of Concrete at $57.5 \mathrm{GHz}$," in IEEE Trans. on Antennas and Propagation. Vol. AP-44(1), January, 1996, pp. 35-40.

W.R. Scott, Jr. and G.S. Smith, 1992, "Measured Electrical Constitutive Parameters of Soil as Functions of Frequency and Moisture Content," in IEEE Trans. on Geoscience and Remote Sensing. Vol. GE-30(3), May, 1992, pp. 621-623.

G.C. Sherman and K.E. Oughstun, 1981, "Description of Pulse Dynamics in Lorentz Media in Terms of the Energy Velocity and Attenuation of Time-Harmonic Waves," in Physical Review Letters. Vol. 47(20), 16 November 1981, pp. 1451-1454.

W. Su, R. Mostafa, S.M. Riad, and I.L. Al-Qadi, 1996, "Characterization of Concrete Material Using TEM Horn Antennas." Presented at the URSI National Radio Science Meeting, Boulder, CO, January 11, 1996. Bradley Dept. of Electrical Engineering, Virginia Polytechnic Institute and State University, Blacksburg, VA.

A.R. von Hippel, ed., 1954, Chs. 1 and 2 in Dielectric Materials and Applications. Tech. Press of MTT and Wiley, New York.

J.R. Wait, 1969, "Electromagnetic Fields of Sources in Lossy Media," Ch. 24 in Antenna Theory, Part 2, R.E. Collin and F.J. Zucker, eds. McGraw-Hill, New York.

J.R. Wang and T.J. Schmugge, 1980, "An Empirical Model for the Complex Dielectric Permittivity of Soils as a Function of Water Content," in IEEE Trans. on Geoscience and Remote Sensing. Vol. GE-18(4), October, 1980, pp. 288-295. 


\section{APPENDIX}

\section{TEM Horn Antenna with Dielectric Lens}

\section{Introduction}

This Appendix describes the new TEM horn antennas which we specifically designed and built for the Series-2 propagation measurements. They incorporate a dielectric aperture lens in order to achieve faster transient pulse response. TEM (transverse electromagnetic) horns are commonly used for wideband time-domain work because they offer minimum dispersion as a traveling-wave endfire structure (which can be made fairly nonresonant). However, even carefully designed TEM horns have an inherent pulse-smearing geometrical effect due to spherical wavefront propagation within the structure. A dielectric planar-convex aperture lens is used to accomplish this plane-wave to spherical-wave conversion, by collimating the wavefront between the plates in order to improve the impulse response. We also required antennas with greater main-beam gain or sensitivity than the simple TEM horns used in the Series-1 propagation experiments.

The antenna consists of a conventional TEM horn configuration (two flat, long triangularly-shaped conducting plates with a constant separation angle), and an additional solid Teflon ${ }^{\mathrm{TM}}$ lens placed at the aperture end of the plates. A $1-\mathrm{m}$ long antenna was designed and built. Two different schemes were employed for the plate configuration: the first version utilizes single-sided etched copper traces on low-loss printed-wiring boards; and the other version utilizes solid copper plates. In both configurations expanded polystyrene is employed as a solid structural supporting material between the plates, and the dielectric planar-convex lens is located at the aperture end of the plates.

The printed-board configuration is designed with stepped resistive loading at the aperture end of the traces in order to minimize ringing antenna currents, and a custom transition from the parallel-plate antenna structure to coaxial feedpoint. The solid-plate configuration was then developed because the impulse response of the printed-board topology wasn't good enough. The resulting step-equivalent risetime (10-90\%) of the solid-plate version is $20 \mathrm{ps}$, the fastest TEM horn we have designed and built to date. This paper describes our antenna design for both plate configurations, and measurements of the resulting performance for two nominally identical antennas. This type of antenna offers very good short-pulse operation, and is highly recommended for wideband time-domain antenna work.

\section{Printed-Board Configuration}

The printed-board configuration was the first design effort for our next generation of time-domain antennas, incorporating two primary features: a planar-convex dielectric lens to collimate the internal spherical-wave behavior of a TEM horn, resulting in much faster transient 
response (for either transmit or receive operation); and resistive loading on the conducting plates of the TEM horn in order to reduce the undesired ringing of antenna currents between the feedpoint and the aperture.

Figure A-1 shows one of two identically-built TEM horn antennas which utilizes the printed-board construction. Most of the body consists of solid expanded polystyrene, with a dielectic constant very close to free-space (for minimal perturbation of the electromagnetic fields in the antenna structure). The lens is seen positioned between the aperture end of the two printed-wiring boards, with single-sided copper traces on the outside surfaces of the two boards. The aperture plate (and lens) width is $30.5 \mathrm{~cm}$, and the plate separation (lens height) is $12.7 \mathrm{~cm}$. The length of the flat-plate conductors is $80.0 \mathrm{~cm}$ from the focal point to the center of the aperture end of each conductor. The resulting plate-separation angle is $9^{\circ}$, and the plate-width angle is $22^{\circ}$. Because the primary goal was to minimize the duration of the time-domain impulse response, the plate-separation angle is shallow compared to typical TEM horn designs of $15-20^{\circ}$. The surge impedance along the antenna was allowed to increase from the feedpoint level of $50 \Omega$ up to about $95 \Omega$ at the aperture, in order to increase the main-beam transmit gain and/or receive sensitivity with a given aperture width.

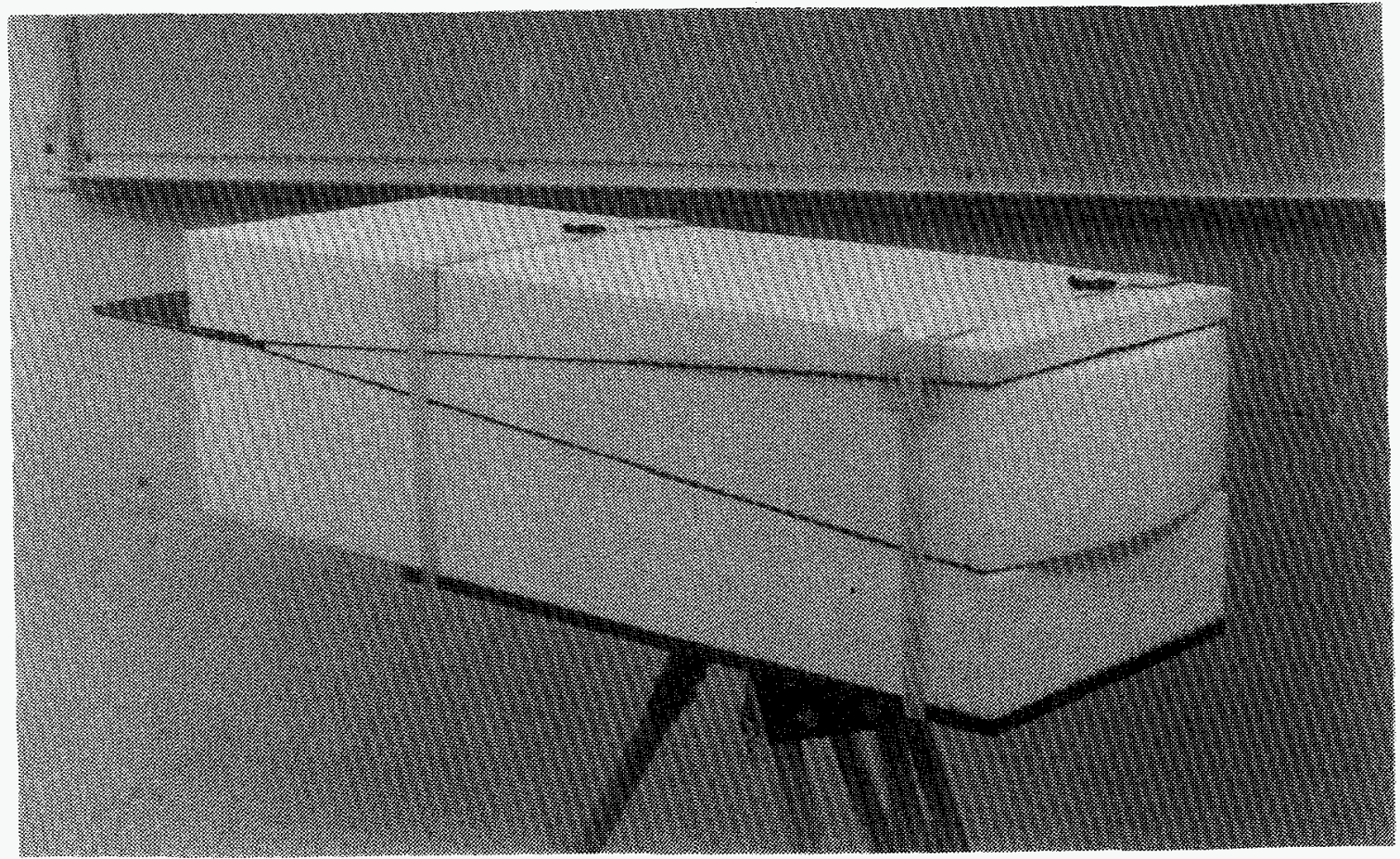

Figure A-1. Printed-Board Configuration of TEM Horn Antenna with Dielectric Lens.

Figure A-2 shows the top and bottom printed-wiring boards (of the two antennas - one is turned upside down). These boards are Rogers RT/Duroid ${ }^{\mathrm{TM}} 5880$ material, with the lowest dielectric constant and loss commercially available. At the aperture end (or right side) of the boards, there are six rectangular sections of resistive loading, each consisting of nichrome thin-film material. Their surface resistivity increases in a stepped exponential fashion to approximate a continuously-increasing loss to the antenna plate currents. 


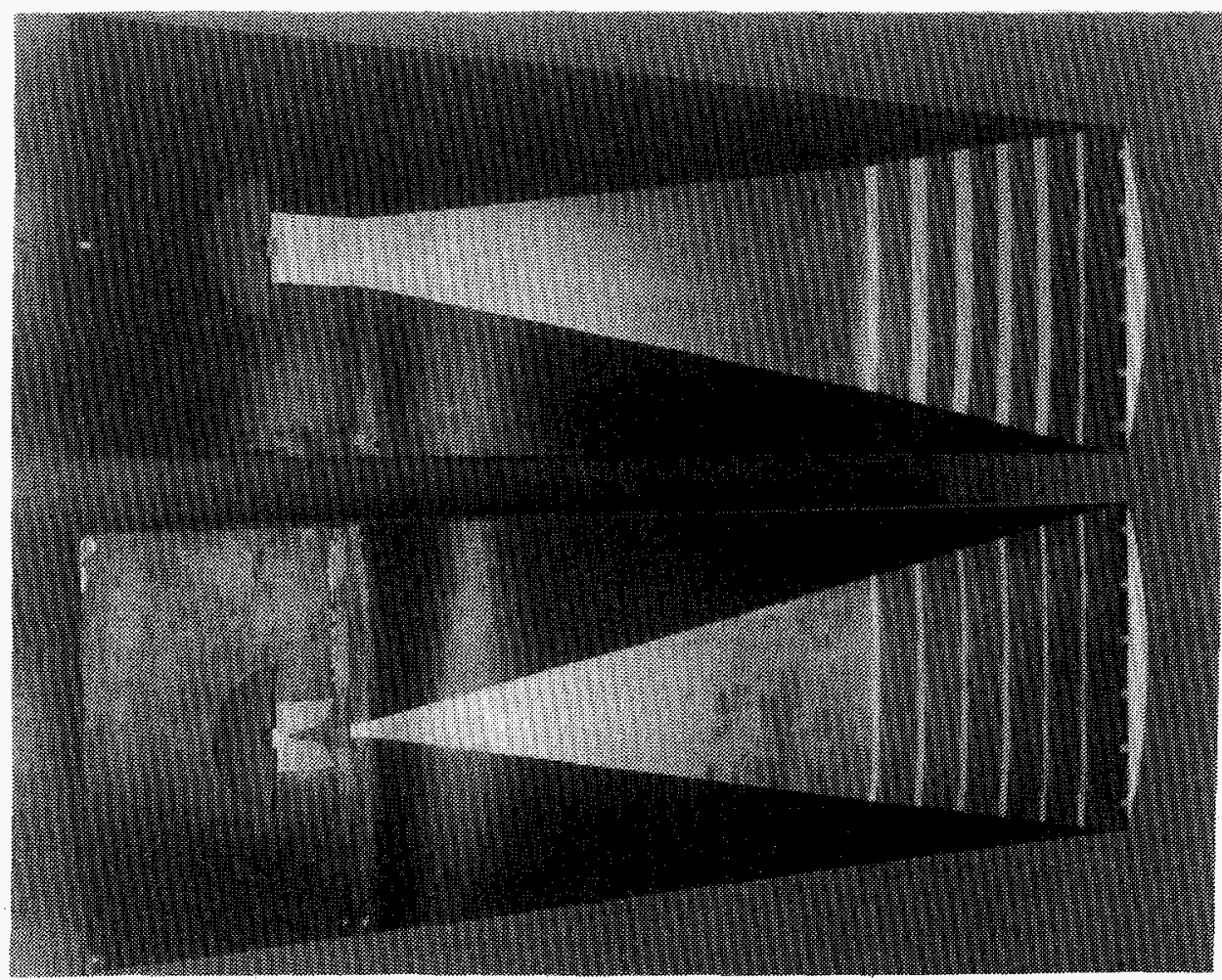

Figure A-2. Top Surface (lower antenna shown) and Bottom Surface of Printed-Board Configuration of TEM Horn Antenna.

At the feedpoint region the bottom antenna has a small butterfly-shaped copper trace which is an integral portion of the custom transmission-line transition from the endfire-coax input (SMA female) to the balanced parallel-plate cross-section of the antenna proper. This transition was carefully designed and is a unique succession of endfire coax, balanced stripline, unbalanced microstrip, and finally balanced parallel-plate topology for most of the antenna flared region. It was expressly designed to provide a smooth transition from the coaxial port to balanced antenna currents on the antenna plates, with minimal variation in surge impedance through the feed transition region.

The Teflon ${ }^{\mathrm{TM}}$ (polytetrafluoroethylene) lens was designed with straightforward geometrical equal-path ray tracing, with a planar inner face and a convex aperture or outer face. Then a 3D spherical curve-fit was performed so that the resulting planar-spherical lens could be easily made on a $\mathrm{CNC}$ machine.

After two nominally identical printed-board antennas were assembled, the time-domain radiation performance was measured in a boresight transmit-receive configuration with step generator and $20-\mathrm{GHz}$ sampling oscilloscope. Compared to our older TEM horns, this new antenna offered several times better gain or sensitivity, but the step-equivalent risetime was much too slow (on the order of $100 \mathrm{ps}$ ). The increased main-beam gain is directly due to the increased plate separation or aperture height, which increases the aperture area of the antenna. The poor time-domain response is surmised to be due to pulse smearing of the currents in the antenna conductors due to the direct presence of the printed-wiring board dielectric substrate. This has 
the effect of slowing the propagation velocity of the antenna current on the substrate side (inner surface) of the copper traces. On the other hand, the current on the outer/air side of the copper traces propagates at the speed of light, and the resulting combination of these two current components suffers pulse spreading due to different overall propagation times along the antenna plates.

\section{Solid-Plate Configuration}

After we found out that the transient response of the printed-board configuration was too slow, a simpler version was designed and retrofitted into the same polystyrene antenna body. This second configuration consists of solid metal plates in place of the single-sided printed-wiring boards in the attempt to remove the board substrate and its' pulse-spreading effect. Figure A-3 shows both TEM horns, after retrofitting with the metal-plate configuration for the antenna conductors.

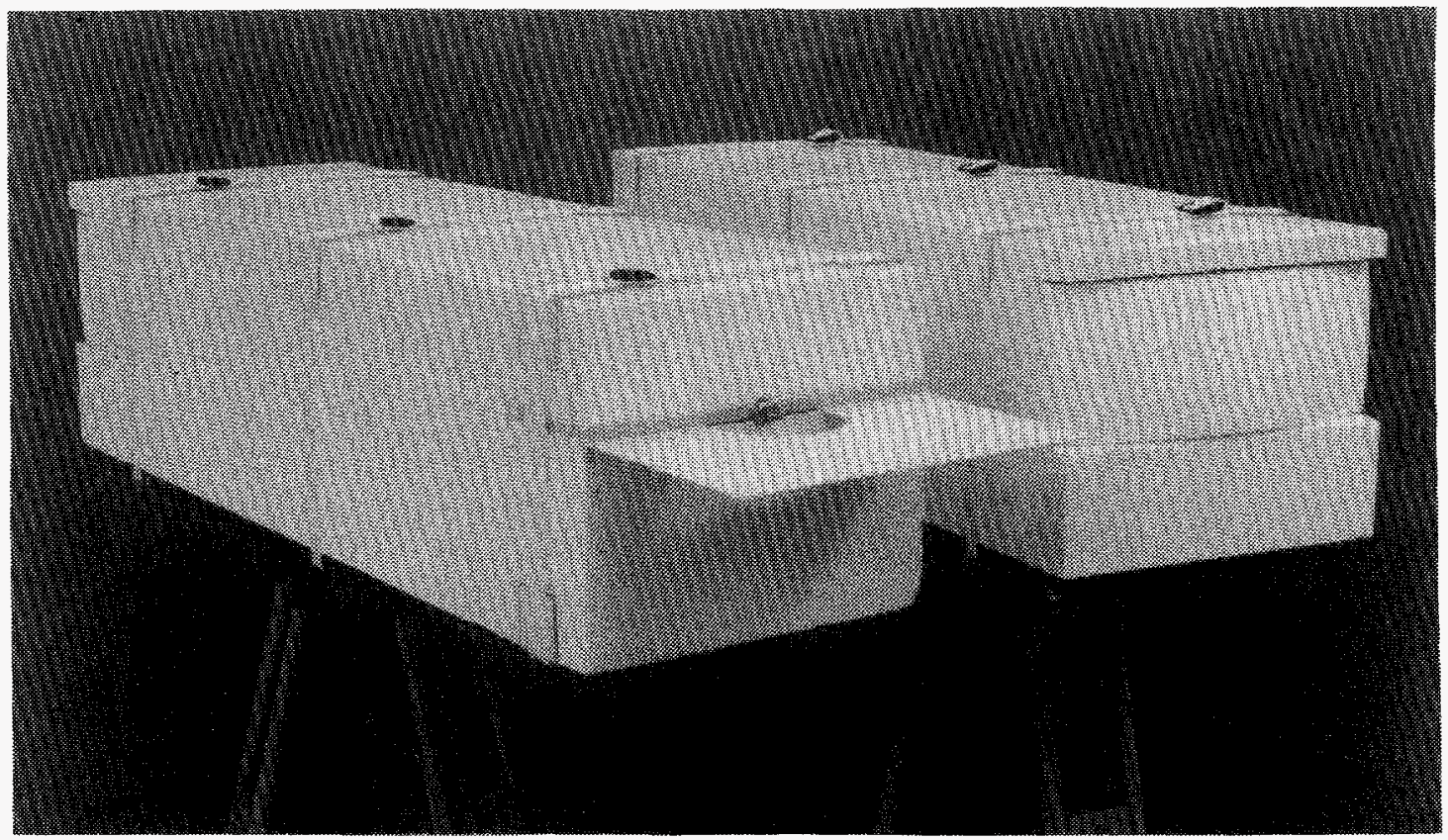

Figure A-3. Solid-Plate Configuration of TEM Horn Antennas.

Figure A-4 shows the top and bottom conductors of the solid-plate configuration, with a very clean geometry. The triangular conductors are full length, with no resistive loading; the lenses are the same ones used for the printed-board configuration. Figure A-5 then shows a close-up view of the feedpoint region. This consists of a simple but carefully constructed transition from transverse-fed coaxial line to unbalanced parallel-plate which then smoothly transforms to the balanced parallel-plate antenna structure. In past antenna design efforts, a variety of coax-to-parallel plate transitions have been constructed and evaluated. This transverse-fed topology has worked the best for short-duration time-domain operation at low voltage levels. Attempts were made to build both antennas with identical feedpoint characteristics, and step time-domain reflectometry was used to empirically adjust the feedpoint conductor spacing in order to have the same surge impedance variation in each antenna. 


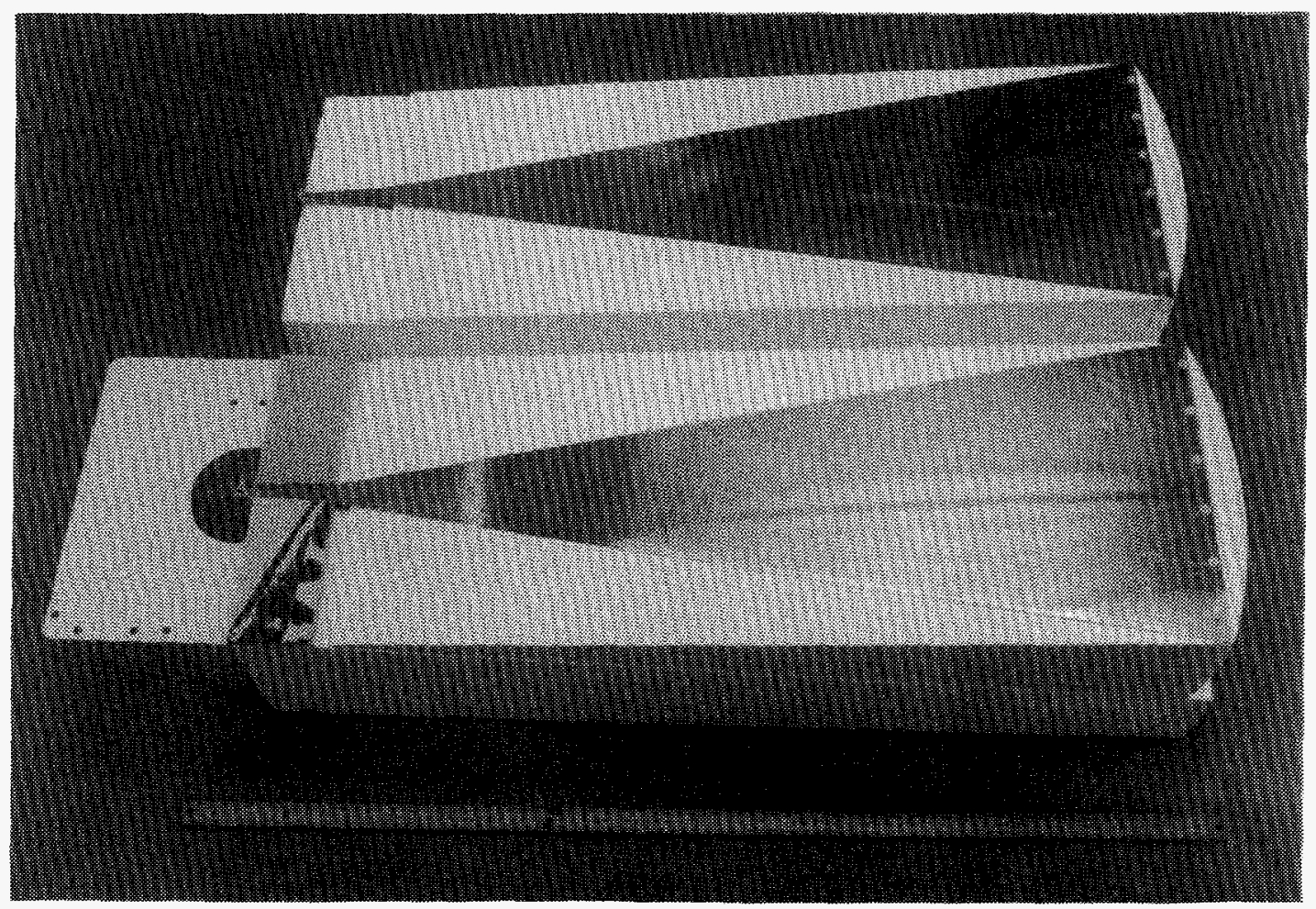

Figure A-4. Top and Bottom Conductors of Solid-Plate Antenna Configuration.

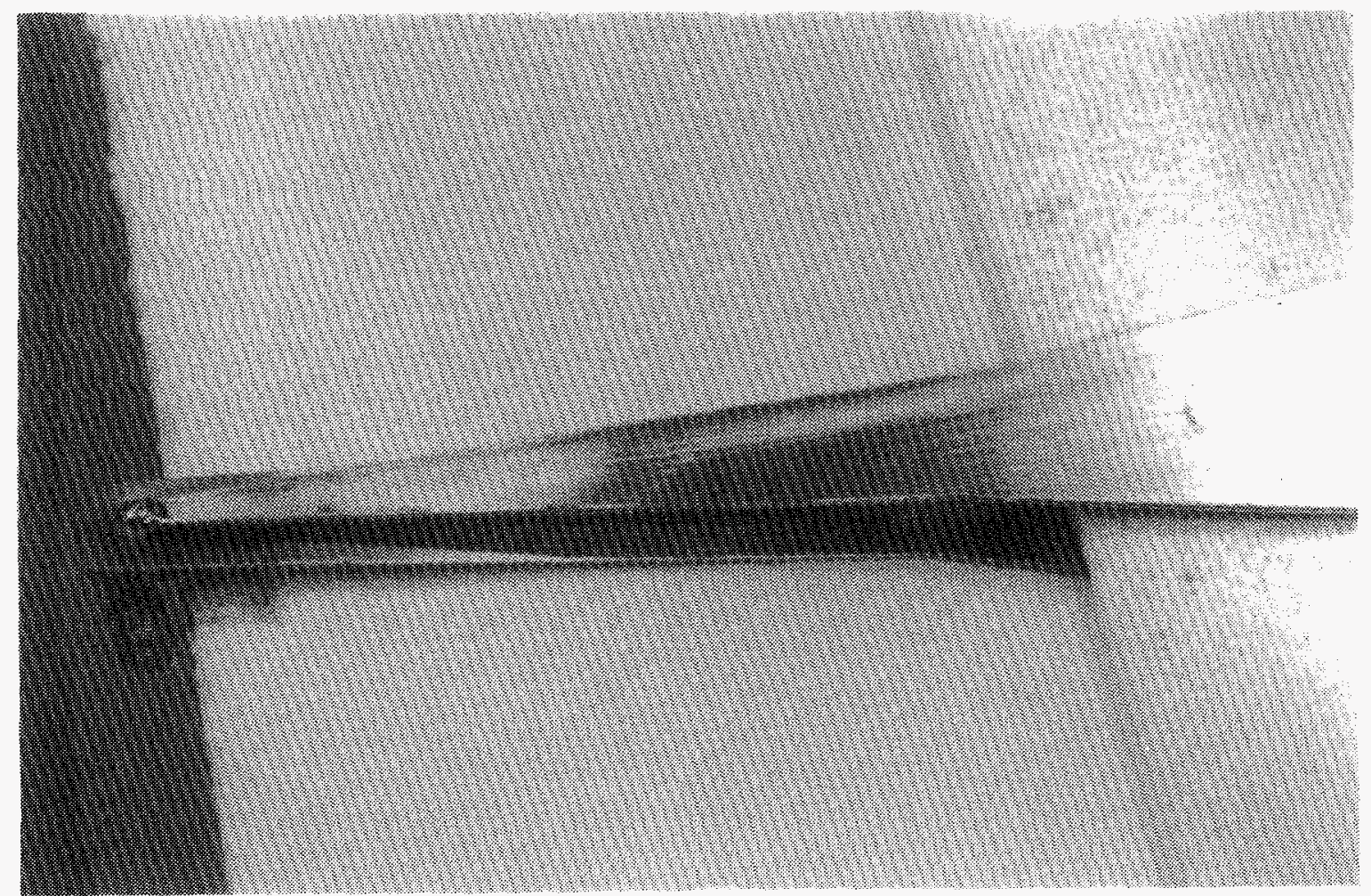

Figure A-5. Close-up View of Feedpoint Region of Solid-Plate Antenna Configuration. 


\section{Measurements of Solid-Plate Antennas}

After we assembled this pair of TEM horn antennas, they were extensively used in the Series-2 propagation measurements. Then after this experimental program was over, we were interested in more carefully measuring the time-domain response of the transmit-receive antenna combination. Figure A-6 shows the indoor antenna setup, with both TEM horns boresight aligned for main-beam response. The transmitting apparatus is at the rear of the photo, aimed at the receiving equipment, which is nearest to the camera. The aperture separation was $2.00 \mathrm{~m}$, and the antenna centerline height above the lab floor was $1.75 \mathrm{~m}$.

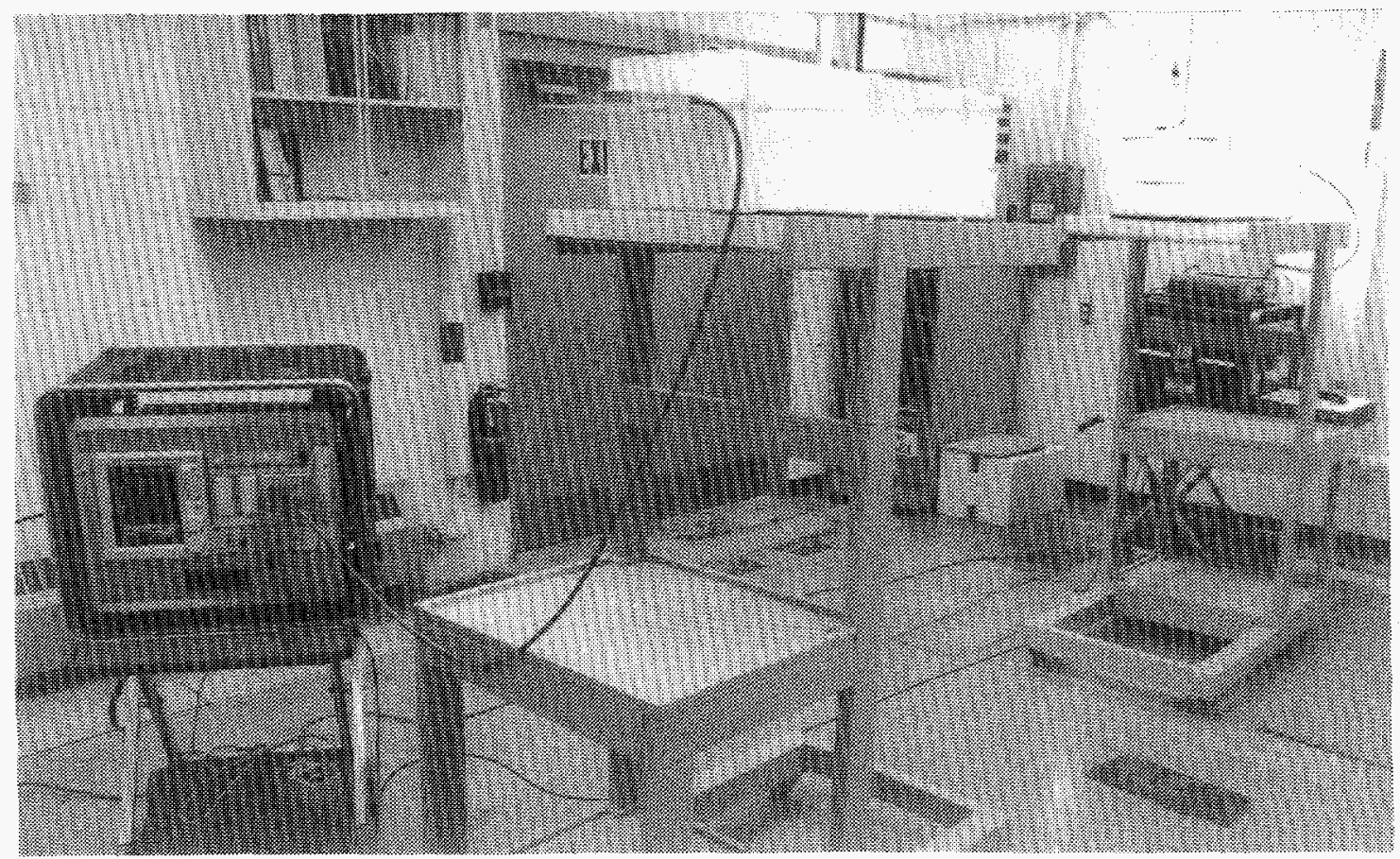

Figure A-6. Experimental Setup to Measure Boresight Time-Domain Response of Solid-Plate Antenna.

Figure A-7 is a block diagram of the system hardware used for the boresight time-domain measurement. A very fast step pulse is generated by the Picosecond Pulse Labs 4015B generator (by its' remote pulse head), and the transmit antenna radiates a boresight derivative, resulting in an impulse radiated E-field. The receiving system consisted of a Tektronix CSA-803 digital sampling oscilloscope, with an SD-24 20-GHz sampling head located close to the receive antenna. This is one of the fastest free-space measurement systems which we have assembled, and the step response had a $10-90 \%$ transition duration (risetime) of 29 ps.

Figure A-8 shows the two measured waveforms for this characterization. The step waveform is that of the measurement system (without the antennas). The impulse waveform is the received waveform with the double-antenna configuration; this clearly shows the derivative transmitting response of a TEM horn antenna. The receive antenna has a replica response - the time-integral of the transmitting response. Note that both waveforms are normalized to unity amplitude for 
relative comparison. Also the time alignment of the two waveforms is arbitrary, as there is a $2-\mathrm{m}$ propagation delay between the antennas.

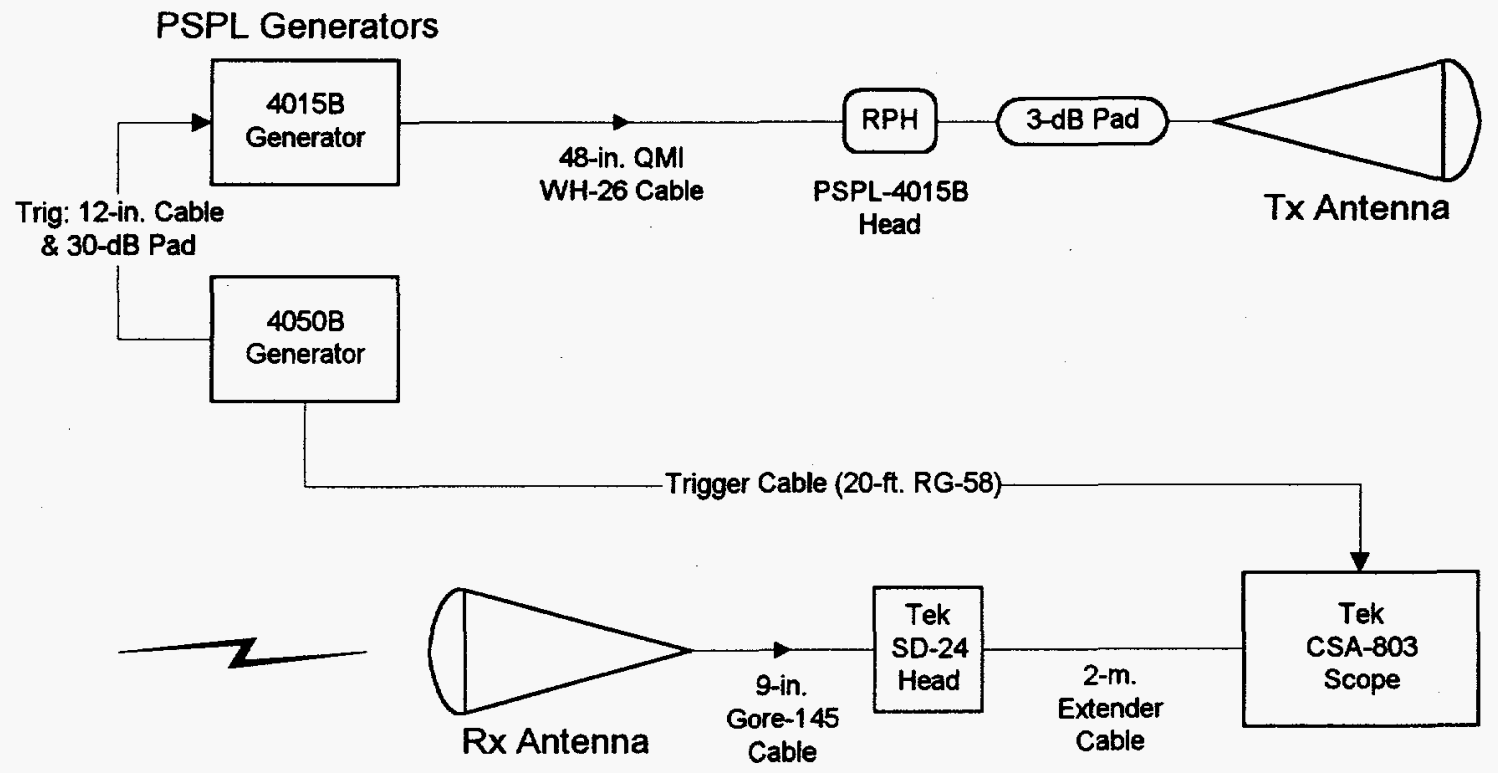

Figure A-7. Block Diagram of Experimental Setup to Measure Boresight Time-Domain Response of Solid-Plate Antenna.

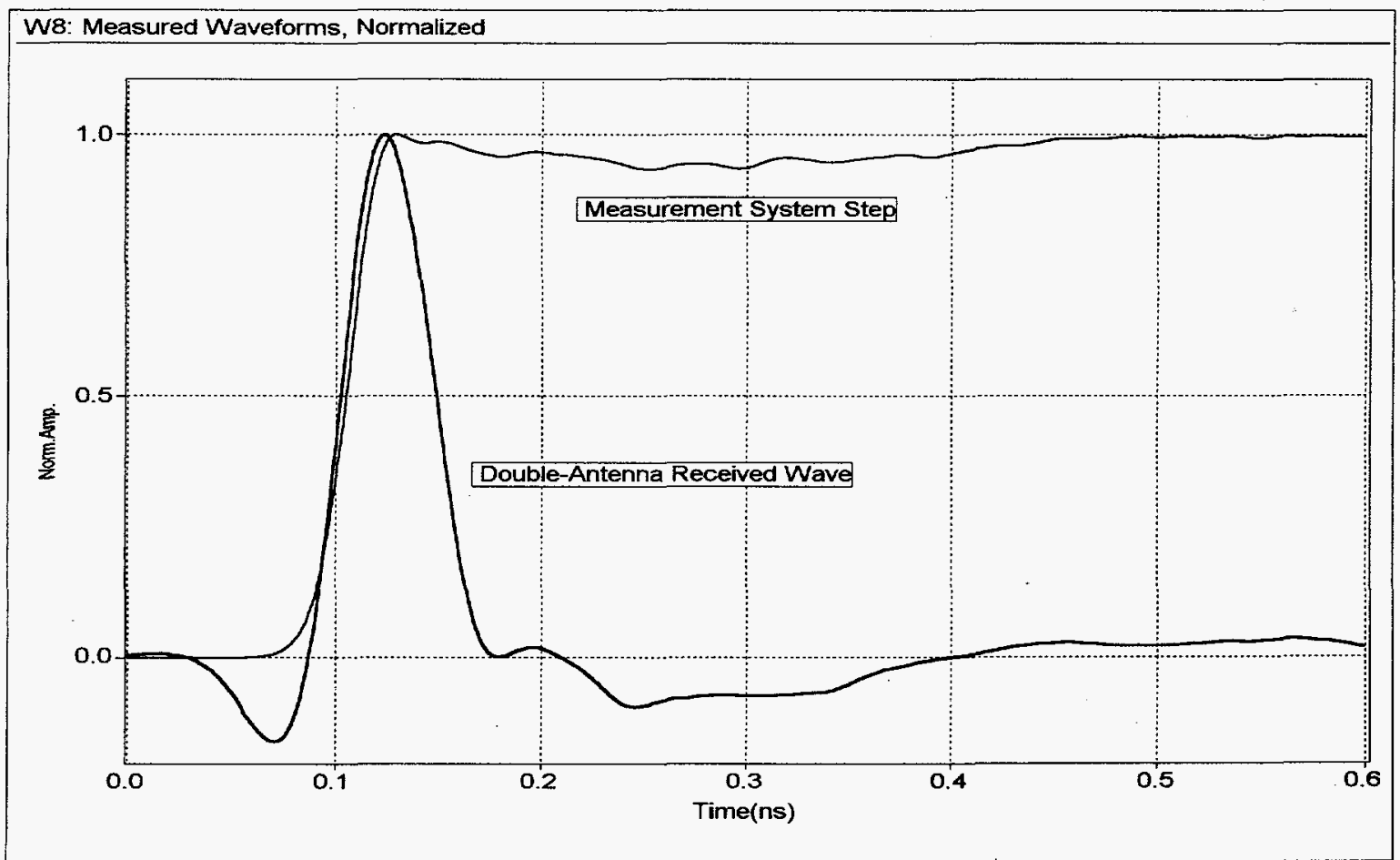

Figure A-8. Boresight Time-Domain Response of Solid-Plate Antenna. 
Figure A-9 shows the same system step waveform and a time-integration of the antenna response waveform. Using simple quadrature analysis of the transition duration (10-90\% risetime) based on the assumption of Gaussian system components, the degradation or slowing of the transition duration can be unfolded from the response risetime. The observed or measured risetime, $t_{r}(m m t)$, is the squared sum of the each antenna's 'step-equivalent' risetime, $t_{r}(a n t)$, and the risetime of the measurement system, $t_{r}(s y s)$ :

$$
t_{r}^{2}(m m t)=2 t_{r}^{2}(\text { ant })+t_{r}^{2}(s y s) \text {. }
$$

This assumes that the two antennas are very similar in radiation performance, which has been verified. Solving for the antenna step-equivalent transition duration,

$$
\begin{aligned}
t_{r}(\text { ant }) & =\sqrt{\frac{1}{2}\left[t_{r}^{2}(m m t)-t_{r}^{2}(s y s)\right]} \\
& =\sqrt{\frac{1}{2}\left[(40.7 \mathrm{ps})^{2}-(28.9 \mathrm{ps})^{2}\right]} \\
& =20.3 \mathrm{ps} .
\end{aligned}
$$

This is the fastest transient response of any time-domain antenna which we have designed and built in our department.

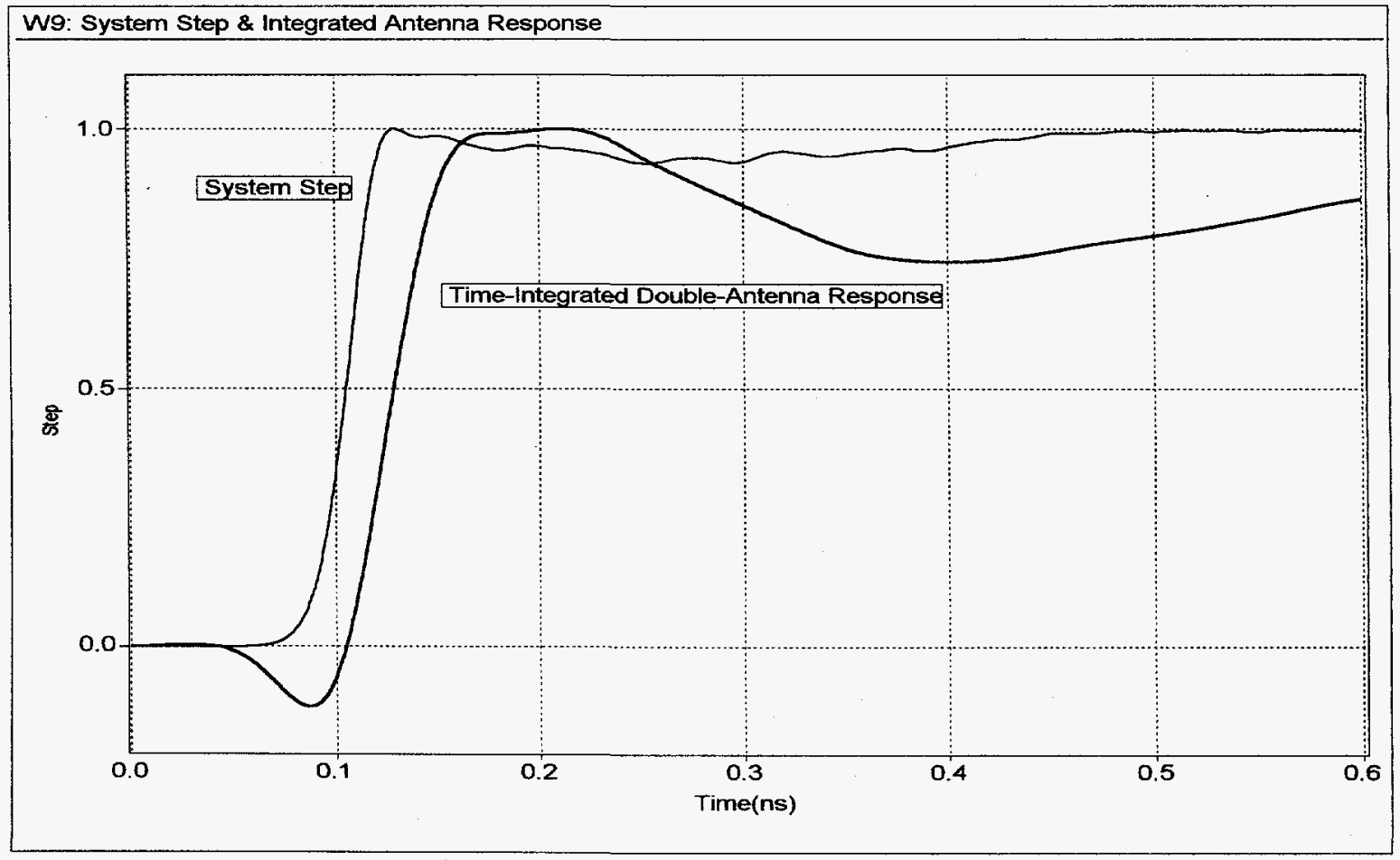

Figure A-9. Time-Integrated 'Step-Equivalent' Boresight Time-Domain Response of Solid-Plate Antenna.

At the same time, we were interested in the pattern drop-off in the horizontal plane because this would affect the possible internal re-bar scattering in the concrete walls at angles off of boresight (normal incidence through the wall). So we measured the H-plane time-domain pattern by rotating the receive antenna. Figure A-10 shows two photos of the measurement setup in our lab, with the transmitter at the rear and the receiver at the front of the figures. The aperture lens 
separation was $1.48 \mathrm{~m}$, and the centerline antenna height above the floor was $1.22 \mathrm{~m}$. The receive antenna was rotated about a centerline point $33 \mathrm{~cm}$ behind the lens front. Figure A-11 is the corresponding block diagram of the measurement system.
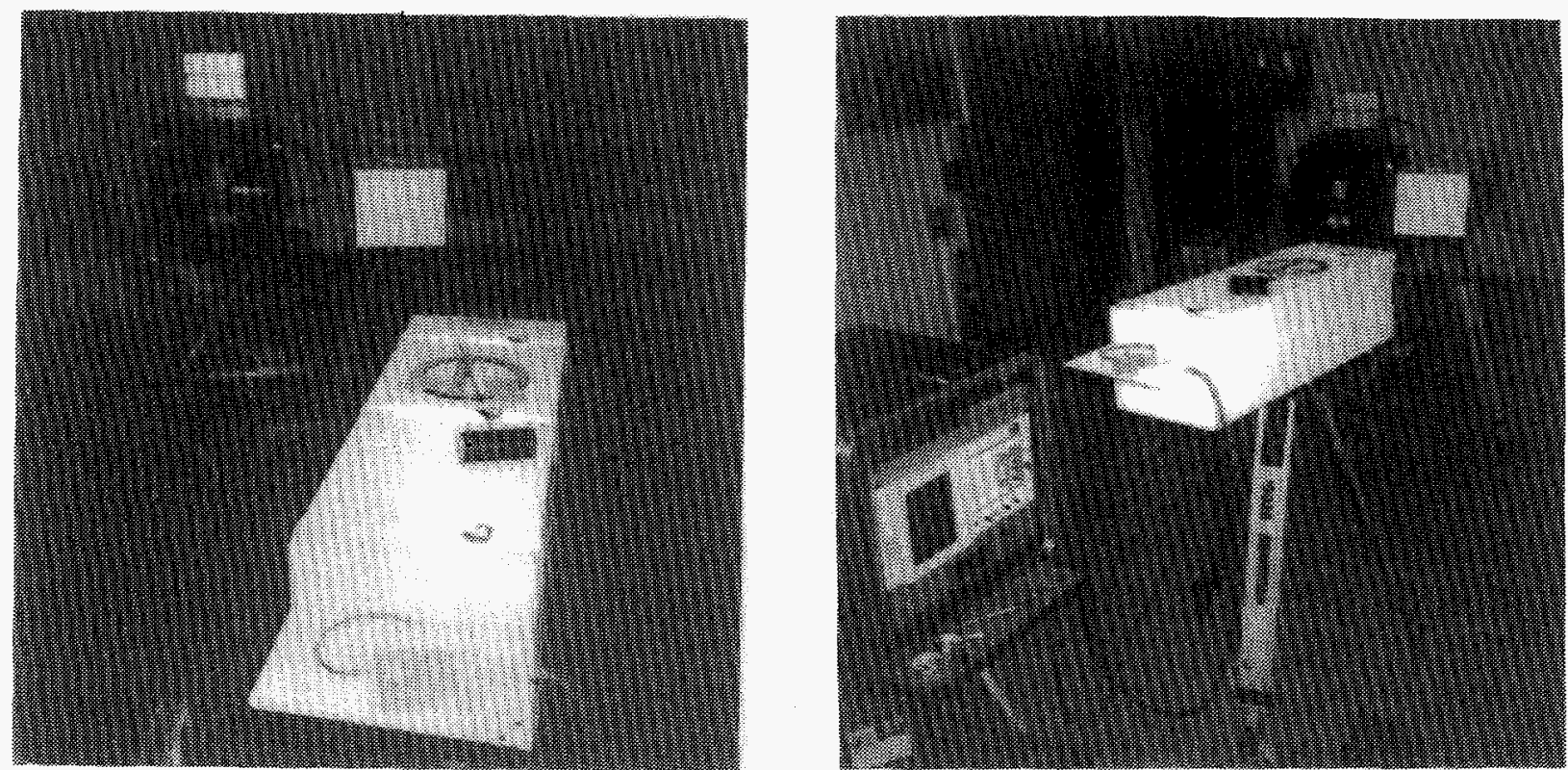

Figure A-10. Experimental Setup to Measure Horizontal (H-Plane) Time-Domain Pattern of Solid-Plate TEM Horn Antenna.

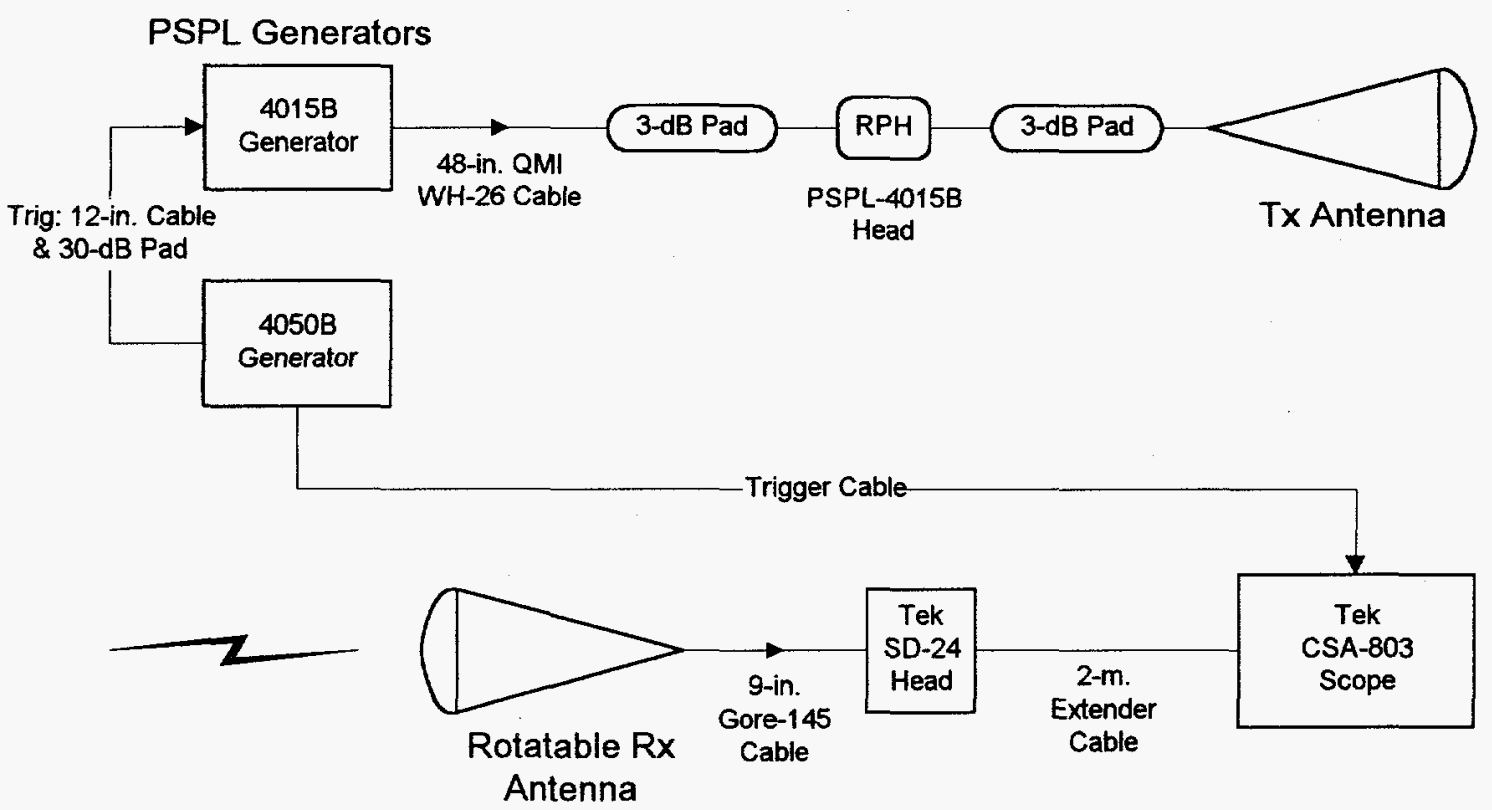

Figure A-11. Block Diagram of Experimental Setup to Measure H-Plane Time-Domain Pattern of Solid-Plate Antenna.

Figure A-12 shows the received waveform versus $\mathrm{H}$-plane angle off boresight, in $5^{\circ}$ increments, from $0^{\circ}$ to $30^{\circ}$. Notice that the primary impulse feature in the main beam is lost beyond $10^{\circ}$, so that propagation scattering features beyond this angle will be excited and received 


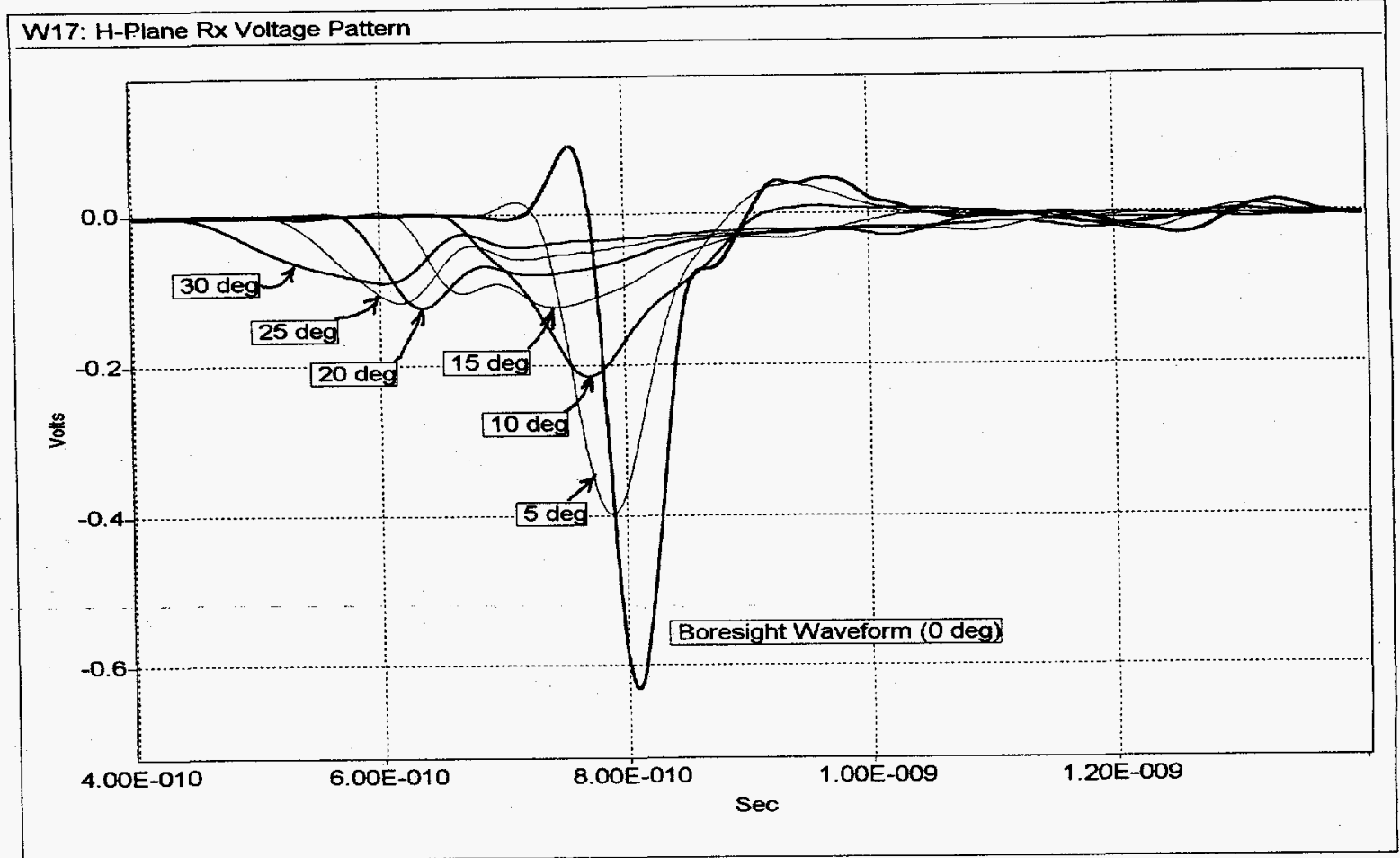

Figure A-12. Received Waveforms for Time-Domain H-Plane Pattern of Solid-Plate Antenna.

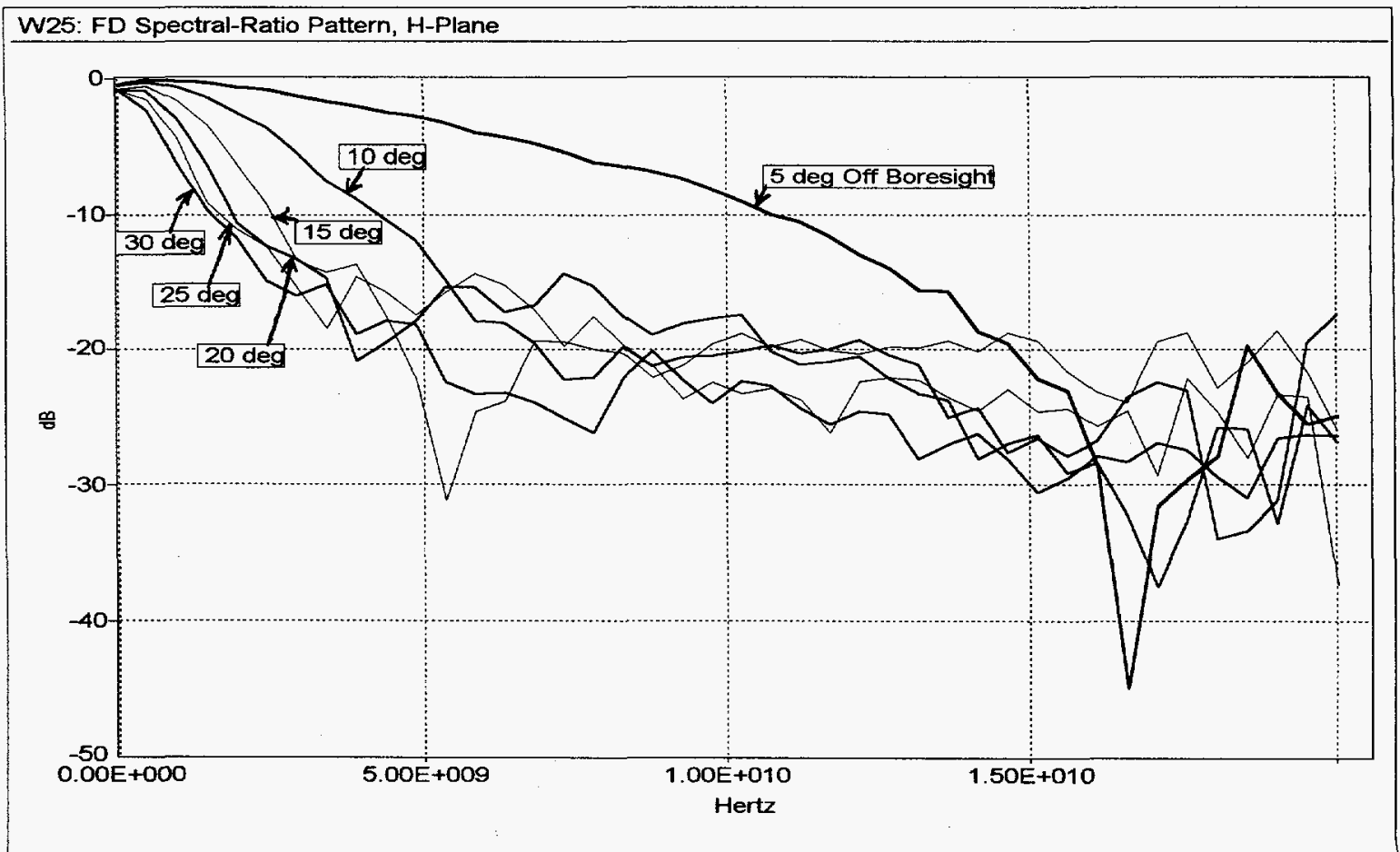

Figure A-13. Frequency-Domain H-Plane Pattern of Solid-Plate TEM Horn Antenna, Based on Spectral Ratio of Received Waveforms in Figure A-12. 
with the smeared-out lower-frequency response in the off-axis 'pattern'. Figure A-13 shows the frequency-domain pattern rolloff with angle, based on the ratio of the Fourier spectrum of these waveforms to that of the boresight $\left(0^{\circ}\right)$ waveform. This is consistent with the time-domain observation - most of the operating bandwidth has fallen off at $\mathrm{H}$-plane angles greater than $10^{\circ}$.

\section{Summary}

We designed and constructed a pair of TEM horn antennas specifically for very fast time-domain boresight response. Two physical topologies were made: a printed-board configuration; and a solid-plate configuration. The printed-board configuration has much slower transient response, which we think is due to pulse-smearing of the antenna currents in the dielectric substrate of the printed-wiring boards. The solid-plate version has a 20-ps transition-duration response in the main-beam endfire (boresight) direction, which is the fastest (highest bandwidth) we have seen to date. And since the antenna has a round-trip antenna-current propagation time of $6 \mathrm{~ns}$, it offers clean radiated electromagnetic-field measurement capability with a clear time of several $\mathrm{ns}$. This was intentionally designed for propagation measurements through walls of 1 to $2 \mathrm{ft}$. thickness. The printed-board version has resistive loading at the aperture end of the conductors, which should offer better low-frequency performance (time has not permitted studying this issue however). The dielectric lens certainly does improve the transient performance of the TEM horn, and was simple to design. The solid-plate version of the antenna pair were used for the Series-2 propagation experiments. In the future we hope to study possible enhancements to either or both configurations. 
Blank Page 


\section{DISTRIBUTION}

1 James Andrews

Picosecond Pulse Labs

P.O. Box 44

Boulder, CO 80306

1 David Falconer

SRI International

MS 301-64

333 Ravenswood Ave.

Menlo Park, CA 94025

1 Everett Farr

Farr Research

614 Paseo Del Mar NE

Albuquerque, NM 87123

1 Charles Frost

Pulse Power Physics

1039 Red Oaks Loop NE

Albuquerque, NM 87122

1 Leo Kempel

Mission Research Corp.

147 John Sims Parkway

Valparaiso, FL 32580-1271

1 Ira Kohlberg

P.O. Box 23077

Alexandria, VA 22304

2 National Institute of Standards \& Tech.

Attn: David Hill

Arthur Ondrejka

MS- 813.07

325 Broadway

Boulder, CO 80303-3328
2 Naval Postgraduate School

Attn: Michael Morgan, Code EC/Mw

Clark Robertson, Code EC/RC

Monterey, CA 93943-5000

3 Phillips Laboratory

Attn: Tracey Bowen, WSM

David Dietz, WSM

Robert Gardner, WSM

3550 Aberdeen SE

Kirtland AFB, NM 87117-5776

1 Sedki Riad

Virginia Polytechnic Institute

Bradley Dept. of Electrical Engineering

Blacksburg, VA 24061-0111

3 Wright Laboratory Armament Directorate

Attn: Kwang Min (2) Marcelious Willis, Jr.

WL/MNMF

Eglin AFB, FL 32542-5434

1 Shane Cloude

Applied Electromagnetics Ltd.

5a Alexandra Place

St. Andrews KY16 9XD

SCOTLAND

1 Paul Smith

University of Dundee

Dept. of Mathematics \& Com. Science

Dundee DD1 4HN

SCOTLAND 


\section{DISTRIBUTION (Continued)}

$\begin{array}{lrl}1 & \text { MS } 0533 & \text { Billy Brock, 2343 } \\ 1 & 0533 & \text { Ward Patitz, 2343 } \\ 1 & 0533 & \text { Bill Schaedla, 2343 } \\ 1 & 0533 & \text { Kurt Sorensen, 2343 } \\ 1 & 0529 & \text { Armin Doerry, 2345 } \\ 1 & 0843 & \text { Tom Hitchcock, 2503 } \\ 1 & 0781 & \text { Mark Ladd, 5831 } \\ 20 & 1153 & \text { John Aurand, 9323 } \\ 1 & 1153 & \text { Larry Bacon, 9323 } \\ 1 & 1153 & \text { Malcolm Buttram, 9323 } \\ 1 & 1153 & \text { Gary Denison, 9323 } \\ 1 & 1153 & \text { Guillermo Loubriel, 9323 } \\ 1 & 1153 & \text { Larry Rinehart, 9323 } \\ 1 & 1166 & \text { Doug Riley, 9352 } \\ 1 & 9018 & \text { Central Tech. Files, 8523-2 } \\ 5 & 0899 & \text { Technical Library, 4414 } \\ 2 & 0619 & \text { Review \& Approval Desk, } \\ & & \text { 12630, For DOE/OSTI }\end{array}$

